3. From: (Originating Organization) SWMFE

5. Proj./Prog./Dept./Div.:

SWM

6. Cog. Eigr.:

J. R. Hosser

8. Originator Remarks:

Please sign for approval and release.

4. Related EDT NO.:

N/A

7. Purchase Order No.:

$N / A$

9. Equip./Component No.: $\mathrm{N} / \mathrm{A}$

10. System/Bldg./Facility:

Trenches $31 \& 34 / 218-W-5$

11. Receiver Remarks:

12. Major Assm. Dwg. No.:

N/A

13. Permit/Permit Application No.: N/A

14. Required Response Date:

July 31, 1996

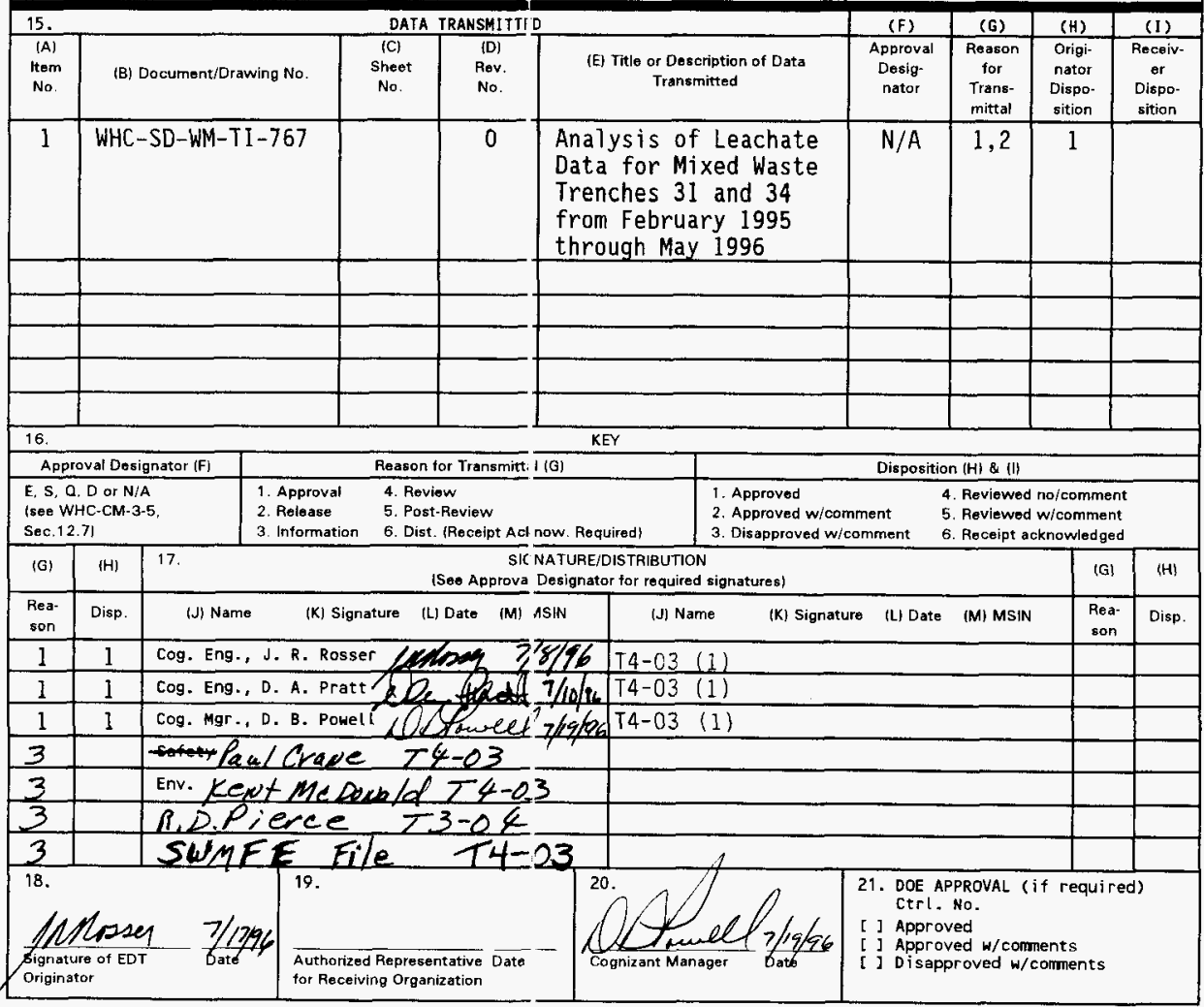




\section{Analysis of Leachate Collection Data for Mixed Waste Trenches 31 and 34 from February 1995 through May 1996}

J. R. Rosser

Westinghouse Handford Co., Richland, WA 99352

U.S. Department of Energy ;ontract DE-ACO6-87RL10930
EDT/ECN: 603188
Org Code: 87250
U:: UC-512
C arge Code: A7VIO
B\&R Code: EW3130020
Total Pages: 46

Key Words: sump, level, lıachate

Abstract: This is an anal'sis of the leachate collection data in burial ground $218-W-5$, trenches 3 and 34 . The data was taken from February 1995 through May 1996.

TRADEMARK DISCLAIMER. Reference here in to any specific commercial product, process, or service by trade name, trademark, manufacturer, or otherwise, does not necessarily constitute or imply its endorsement, recommendation, or favoring by the United States Government or any agency thereof or its contractors or subcontractors.

Printed in the United States of Ameri:a. To obtain copies of this document, contact: WHC/BCS Document Control Services, P.0. Box 1770, Mailstop H6-08, Richland WA 99352, Phone (509) 372-2420; Fax (509) $376-4989$.
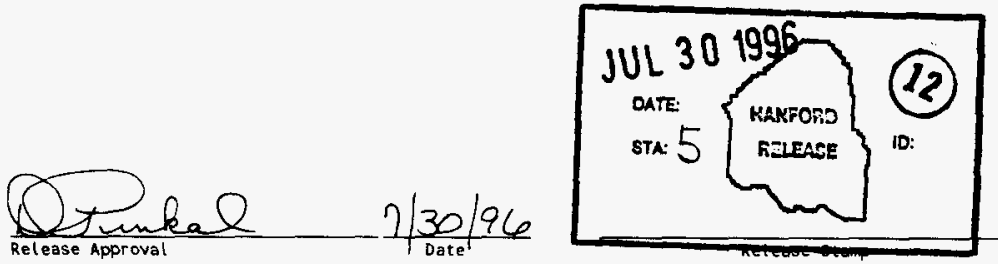

\section{Approved for Public Release}


WHC-SD-WM-TI-767, Rev. 0

Analysis of Leachate Collection Data

for

Mixed V'aste Trenches 31 and 34

from

February 1995 through May 1996 


\subsection{Introduction}

This is an analysis of leachate collection in Burial Ground 218-W-5 Trenches 31 and 34. Data has been taken since Febı uary 13, 1995. The level in both the primary and secondary sumps, along with th $\approx$ amount of leachate pumped from the sumps has been recorded and placed in a spreacsheet format.(See Attachments) In this format, the above recorded data along with precipitation data from the Hanford Weather Station, can be used to help determine the amo ant of leachate coming into each trench and its relationship to the amount of precipitation received at the Hanford site.

\subsection{Scope}

The main focus of this report is to determine how much leachate will have to be pumped out of each trench, stored, samıled, and disposed. But it will also make an effort to determine if the primary liner a d secondary liner in each trench is leaking. This information can be used in a fu ure study to determine if the existing leachate collection system is sufficient for operatio 1 and if the trench liners are performing as desired.

\subsection{Data Uncertainties}

The data, as collected, is sufficient to give an accurate determination (except for one minor problem in Trench 31, which will be noted) of the amount of leachate collected during the period measured. Bit, as will be explained next, it is in some respects suspect and incomplete and it is in this regard that it will not give a definitive answer to if the liners have a leak. The problems with the data include:

Inaccurate leachate flow total for Trench 31 due to a pump failure that required a portable pump that bypassed the flow meter. For the days this was done an estimate was made.

- Level of the sump recolded too soon after pumping, not allowing the leachate to settle and give an accur ite reading.

- Recording sump level r rading then recording flowmeter reading after pumping.

- Long periods where the data was not recorded.

- Possibility of water beir g added to the system for instrument calibration, but not being noted in the logbc ok, for example, Trench 34 on June 26, 1995, showed an increase in both the : econdary flowmeter and sump of over $8,000 \mathrm{gal}$. This would indicate a very la rge leak, which is not indicated anywhere else.

- Level readings in the sumps (especially the secondary) could have been affected (a) by instrument noise b) atmospheric pressure changes which would cause leachate to rise or lowes in the pipe connected to the pump (c) and the depth of water on the primary lirer and the length of time it was allowed to stay there would apply more head pressure to the soil below it and literally squeeze the water, left in that soil level from construction, into the sump. 


\subsection{Data Analysis}

\subsection{Leachate Collection}

In this analysis, numbers in brac sets will be for Trench 34 and those not in brackets will be for Trench 31. The precipita ion data collected, is from February 12, 1995, through May 31, 1996, and the total estinated rain or snow that fell into each trench is $1,161,648 \mathrm{gal}$.

1. Total leachate collected rom February 13, 1995, to May 31, $1996-396,264$ gal. $[412,229 \mathrm{gal}$.$] . Three contributing factors to the amount of leachate$ collected in Trench $34 \mathrm{~b}$ :ing greater than that collected in Trench 31 are (a) Trench 34 possibly havir g a slightly larger surface area (b) Trench 34 was built after Trench 31 and mor 2 water was still captured in the soil from construction and (c) Trench 31 has hed an undetermined amount of leachate (although an estimate has been made :rom Operator reports) pumped out that bypassed the flow meter.

2. The amount of leachate rollected for every $100 \mathrm{gal}$. of precipitation between February 13, 1995, and May 31, 1996 was - 34.2 gal. [35.5 gal.].

It was noted that during lifferent seasons of the year the amount of leachate collected for every 100 吕. of precipitation changed.

- March, 1995, th ough May, 1995 -

Total leachate crllected: 92,765 gal. [80,196 gal.].

Leachate per $10(\mathrm{gal}$. of precip: $34.7 \mathrm{gal}$. [30.0 gal.].

- June, 1995, thro igh August, 1995 -

Total leachate ccllected: 19,427 gal. [37,672 gal.].

Leachate per 10( gal. of precip: 20.2 gal. [39.2 gal.].

- September, 1995, through November, 1995 -

Total leachate ccllected: $10,117 \mathrm{gal}$ [ [8363 gal.].

Leachate per $10($ gal. of precip: $4.6 \mathrm{gal}$. [3.8 gal.].

- December, 1995 through February, 1996 -

Total leachate ccllected: $163,437 \mathrm{gal}$. [176,874 gal.].

Leachate per $10 \mathrm{gal}$. of precip: $40.4 \mathrm{gal}$. [43.8 $\mathrm{gal}$.].

- March, 1996, thu ough May, 1996 -

Total leachate ccllected: 94,139 gal. [78,029 gal.].

Leachate per $10 \mathrm{C}$ gal of precip: $61.5 \mathrm{gal}$. [50.9 gal.].

It is apparent from this comparis on, that evapotranspiration is a large factor in preventing precipitation from becoming leachate in the summer and fall seasons, where as in the winter and spring seaso is a large percentage of precipitation becomes leachate. It should also be noted that the existing 10,000 gal. leachate storage tank for each trench would have filled up between 16 and 17 times during the 90 day period from December 1, 1995 to February 29, 1996. This comes out to having to empty the tank very 5 to 6 days. It should also be noted that if the 24 hour $/ 25$ year storm happened during this period about $127,000 \mathrm{gal}$. of preripitation would enter each trench. At about $40 \mathrm{gal}$. of 
leachate per $100 \mathrm{gal}$. of precipit titon, $50,800 \mathrm{gal}$. of leachate would have to be collected from that storm. If the 24 hour '25 year storm happened in the March through May, 1995 time period approximately $76,200 \mathrm{gal}$. of leachate would be collected from the storm.

\subsection{Leakage Indication}

1. There are two methods o check the possibility of the primary liner leaking with the existing data. Meth $x$ one would have the primary sump level decreasing with no increase in the primary flow from pumping the sump. Method two would have an increase in the secondary sump level.

For both Trenches 31 and 34 there was no indication from Method one that there was a leak in the jurimary liner. Method two did show some indication of possible primary liner leakage in both trenches. But in all cases (except for June 26,1995 , as noted in the Uncertainties section) this increase was very gradual and could have come fr $/ \mathrm{m}$ existing water caught in the soil that was being squeezed out by pressur $\approx$ from the primary liner. In fact this is indicated by the constant high level of the primary sump, therefore more pressure, during these periods.

2. There is one method to sheck the possibility of the secondary liner leaking with the existing data. This would be a decrease in the secondary sump level with no increase in the secondary flow from pumping.

For both Trenches 31 and 34 there were several periods where there was apparently a slow leak c ccurring. During these periods a very gradual decrease the secondary sump occ.urred. This was more noticeable in Trench 34 because the level reading for the sump goes into the hundredths of an inch where the Trench 31 reading goes only to a tenth of an inch. Two indications argue against these gradual de reases as coming from a leak. The first is that the decrease stops and begir's rising gradually. The second is that in most instances both trenches decrease c uring the same or close to the same time frame. This could have been caused by atmospheric pressure changes mentioned in the Uncertainties section.

It is apparent from the existing lata that, although one or more of the above liners could have a leak, the probability is, t tat at this time, none of the liners are leaking. If there is a leak in any liner, it is so in ignificant, it can not be measured with any certainty. 
5.0 Conclusions and Recommendatic ns

5.1 Conclusions

1. During certain times of he year the existing 10,000 gal. leachate storage tank is insufficient to store leaciate for more than an average of five days and maintain the regulatory requireme nt of maintaining less than 12 inches of leachate depth above the liner. Twelve inches of leachate above the liner is about $10,000 \mathrm{gal}$.

2. A 24 hour $/ 25$ year storn could produce over 76,000 gal. of leachate. Obviously this would outstrip the c.apacity of the 10,000 gal. storage tank immediately.

3. Leachate collected in the sump ranges from less than $5 \%$ to greater than $60 \%$ of the total rainfall into the trench, depending on the time of year.

\section{$5.2 \quad$ Recommendations}

1. Engineering study be done to evaluate the alternatives to handle leachate.

2. Devise and run test to cleck if liners are leaking. 
WHC-SD-WM-TI-767, Rev. 0

Appendix A

Glossary

A-1 
Sf readsheet Glossary

1. DATE- Day data was recorded (or in many instances not recorded)

2. Pri. Level inches- $\quad$ Reading in inch:s of leachate level in primary sump.

3. Pri. Level gallons- Chart conversio 1 of primary sump level from inches to gallons.

4. Pri/Sec total flow- Flow meter rearling, in gallons, of total leachate pumped from primary and secondary s.umps.

5. Pri Flow daily- Amount, in gall ons, of leachate pumped out of primary sump that day.

6. Sec Flow total- Flow meter rearling, in gallons, of total leachate pumped from secondary sump.

7. Sec Flow daily- Amount, in gall ons, of leachate pumped out of secondary sump that day.

8. Sec Level inches- Reading in inch ss of leachate level in secondary sump.

9. Sec Level gallons- Chart conversio 1 of secondary sump level from inches to gallons.

10. Total Daily Water-gal- Total at nount of leachate, in gallons, that has entered both the primary and secondary sumps since last data taken. (This would be a daily amount if data was taken daily.)

11. Daily Rain inches- Rainfall for that day, in inches, recorded from Hanford Meteorology Station.

12. Daily Rain gallons- Amount of rain in gallons, that fell into the trench that day.

13. Tot Accu Water-gal- Running total (f Total Daily Water ( $\# 10$ above) in gallons.

14. Tot Accu Rain-gal- Running total o: Daily Rain (\#12 above) in gallons. 
WHC-SD-WM-TI-767, Rev. 0

Appendix B

Tren h 31 Data and Graphs

B-1 


\begin{tabular}{|c|c|c|c|c|c|c|c|c|c|c|c|c|c|}
\hline DATE & \begin{tabular}{|l|} 
Pri. Level \\
inches
\end{tabular} & \begin{tabular}{|l|} 
Pri. Level \\
gallons \\
\end{tabular} & \begin{tabular}{|l|} 
Pri/Sec \\
total flow
\end{tabular} & \begin{tabular}{|l} 
Priflow \\
daily
\end{tabular} & $\begin{array}{l}\text { Sec Flow } \\
\text { total }\end{array}$ & $\begin{array}{l}\text { Sec Flow } \\
\text { daily }\end{array}$ & \begin{tabular}{|l|} 
Sec Level \\
inches
\end{tabular} & \begin{tabular}{|l|} 
Sec Level \\
gallons
\end{tabular} & \begin{tabular}{|l|} 
Total Dailyy \\
Water-gal
\end{tabular} & \begin{tabular}{|l|} 
Daily Rain \\
inches
\end{tabular} & \begin{tabular}{|l|} 
Daily Rain \\
gallons \\
\end{tabular} & \begin{tabular}{|l|} 
Tot Accu \\
Water-gal
\end{tabular} & \begin{tabular}{|l|} 
Tot Accu \\
Rain-gal
\end{tabular} \\
\hline & 16.8 & 1354 & 59246 & & 16919 & & 4.6 & 23 & & 0.23 & 18736.26 & & 18736.26 \\
\hline $2 / 13 / 95$ & 26.9 & 3881 & 60765 & 1519 & 16919 & 0 & 4.6 & 23 & 4046 & & 0 & 4046 & 18736.26 \\
\hline $2 / 14 / 95$ & 29.6 & 4919 & 61015 & 250 & 16919 & 0 & 4.6 & 23 & 1288 & & 0 & 5334 & 18736.26 \\
\hline $2 / 15 / 95$ & 13.4 & 828 & 65231 & 4216 & 16919 & 0 & 4.6 & 23 & 125 & & 0 & 5459 & 18736.26 \\
\hline $2 / 16 / 95$ & 12.8 & 747 & 66129 & 898 & 16919 & 0 & 4.6 & 23 & 817 & & 0 & 6276 & 18736.26 \\
\hline $2 / 17 / 95$ & 15.8 & 1195 & 66799 & 670 & 16919 & 0 & 4.5 & 23 & 1118 & & 0 & 7394 & 18736.26 \\
\hline $2 / 18 / 95$ & 15.8 & 1195 & 66799 & 0 & 16919 & 0 & 4.5 & 23 & 0 & 0.03 & 2443.86 & 7394 & 21180.12 \\
\hline $2 / 19 / 95$ & 15.8 & 1195 & 66799 & 0 & 16919 & 0 & 4.5 & 23 & 0 & & 0 & 7394 & 21180.12 \\
\hline $2 / 20 / 95$ & 15.8 & 1195 & 66799 & 0 & 16919 & 0 & 4.5 & 23 & 0 & & 0 & 7394 & 21180.12 \\
\hline $2 / 21 / 95$ & 29.3 & 4798 & 67190 & 391 & 16919 & 0 & 4.6 & 23 & 3994 & & 0 & 11388 & 21180.12 \\
\hline $2 / 22 / 95$ & 26.1 & 3601 & 68470 & 1279 & 16920 & 1 & 4.6 & 23 & 83 & & 0 & 11471 & 21180.12 \\
\hline $2 / 23 / 95$ & 12.8 & 747 & 72110 & 3641 & 16919 & -1 & 4.5 & 23 & 786 & & 0 & 12257 & 21180.12 \\
\hline $2 / 24 / 95$ & 18.4 & 1675 & 72110 & 0 & 16919 & 0 & 4.5 & 23 & 928 & & 0 & 13185 & 21180.12 \\
\hline $2 / 25 / 95$ & 18.4 & 1675 & 72110 & 0 & 16919 & 0 & 4.5 & 23 & 0 & & 0 & 13185 & 21180.12 \\
\hline $2 / 26 / 95$ & 18.4 & 1675 & 72110 & 0 & 16919 & 0 & 4.5 & 23 & 0 & & 0 & 13185 & 21180.12 \\
\hline $2 / 27 / 95$ & 22.2 & 2496 & 73718 & 1608 & 16919 & 0 & 4.5 & 23 & 2429 & & 0 & 15614 & 21180.12 \\
\hline $2 / 28 / 95$ & 25 & 3261 & 73718 & 0 & 16919 & 0 & 4.5 & 23 & 765 & & 0 & 16379 & 21180.12 \\
\hline $3 / 1 / 95$ & 27.2 & 3991 & 73718 & 0 & 16919 & 0 & 4.5 & 23 & 730 & & 0 & 17109 & 21180.12 \\
\hline $3 / 2 / 95$ & 29.3 & 4798 & 73718 & 0 & 16919 & 0 & 4.5 & 23 & 807 & & 0 & 17916 & 21180.12 \\
\hline $3 / 3 / 95$ & 31.3 & 5686 & 73718 & 0 & 16919 & 0 & 4.5 & 23 & 888 & & 0 & 18804 & 21180.12 \\
\hline $3 / 4 / 40$ & 31.3 & 5686 & 131781 & $\overrightarrow{4}$ & 76979 & 4 & 4.5 & 23 & 0 & & 0 & 18804 & 21180.12 \\
\hline $3 / 5 / 95$ & 31.3 & 5686 & 73718 & 0 & 16919 & 0 & 4.5 & 23 & 0 & & 0 & 18804 & 21180.12 \\
\hline $3 / 6 / 95$ & 27.7 & 4179 & 77098 & 3380 & 16919 & 0 & 4.5 & 23 & 1873 & & 0 & 20677 & 21180.12 \\
\hline $3 / 7 / 95$ & 27.7 & 4179 & 77098 & 0 & 16919 & 0 & 4.5 & 23 & 0 & & 0 & 20677 & 21180.12 \\
\hline $3 / 8 / 95$ & 30.1 & 5126 & 77576 & 478 & 16919 & 0 & 4.5 & 23 & 1425 & & 0 & 22102 & 21180.12 \\
\hline $3 / 9 / 95$ & 29.2 & 4757 & 78690 & 1114 & 16919 & 0 & 4.5 & 23 & 745 & 0.09 & 7331.58 & 22847 & 28511.7 \\
\hline $3 / 10 / 95$ & 19.5 & 1901 & 83265 & 4575 & 16919 & 0 & 4.5 & 23 & 1719 & 0.42 & 34214.04 & 24566 & 62725.74 \\
\hline $3 / 11 / 95$ & 19.5 & 1901 & 83265 & 0 & 16919 & 0 & 4.5 & 23 & 0 & 0.21 & 17107.02 & 24566 & 79832.76 \\
\hline $3 / 12 / 95$ & 19.5 & 1901 & 83265 & 0 & 16919 & 0 & 4.5 & 23 & 0 & & 0 & 24566 & 79832.76 \\
\hline $3 / 13 / 95$ & 36 & 8304 & 83813 & 548 & 16919 & 0 & 4.5 & 23 & 6951 & & 0 & 31517 & 79832.76 \\
\hline $3 / 14 / 95$ & 22.6 & 2596 & 92680 & 8867 & 16919 & 0 & 4.5 & 23 & 3159 & 0.16 & 13033.92 & 34676 & 92866.68 \\
\hline $3 / 15 / 95$ & 19.3 & 1859 & 94717 & 2037 & 16919 & 0 & 4.5 & 23 & 1300 & & 0 & 35976 & 92866.68 \\
\hline $3 / 16 / 95$ & 19.4 & 1880 & 94717 & 0 & 16919 & 0 & 4.5 & 23 & 21 & & 0 & 35997 & 92866.68 \\
\hline $3 / 17 / 95$ & 25.1 & 3292 & 94717 & 0 & 16919 & D & 4.5 & 23 & 1412 & & 0 & 37409 & 92866.68 \\
\hline $3 / 18 / 95$ & 25.1 & 3292 & 94717 & 0 & 16919 & 0 & 4.5 & 23 & 0 & & 0 & 37409 & 92866.68 \\
\hline $3 / 19 / 95$ & 25.1 & 3292 & 94717 & 0 & 16919 & 0 & 4.5 & 23 & 0 & & 0 & 37409 & 92866.68 \\
\hline $3 / 20 / 95$ & 34 & 7164 & 94718 & 1 & 16919 & 0 & 4.5 & 23 & 3873 & 0.07 & 5702.34 & 41282 & 98569.02 \\
\hline $3 / 21 / 95$ & 16.6 & 1334 & 101384 & 6666 & 16919 & 0 & 4.5 & 23 & 836 & & 0 & 42118 & 98569.02 \\
\hline $3 / 22 / 95$ & 22.1 & 2471 & 101385 & 1 & 16919 & 0 & 4.5 & 23 & 1138 & & 0 & 43256 & 98569.02 \\
\hline
\end{tabular}


Trench 31

Sheet1

\begin{tabular}{|c|c|c|c|c|c|c|c|c|c|c|c|c|c|}
\hline DATE & \begin{tabular}{|l|} 
Pri. Level \\
inches
\end{tabular} & \begin{tabular}{|l|} 
Pri. Level \\
gallons
\end{tabular} & \begin{tabular}{|l|} 
Pri/Sec \\
total flow
\end{tabular} & \begin{tabular}{|l} 
Pri Flow \\
daily
\end{tabular} & \begin{tabular}{|l} 
Sec Flow \\
total
\end{tabular} & \begin{tabular}{|l|} 
Sec Flow \\
daily
\end{tabular} & \begin{tabular}{|l} 
Sec Level \\
inches
\end{tabular} & $\begin{array}{l}\text { Sec Level } \\
\text { gallons }\end{array}$ & \begin{tabular}{|l|} 
Total Daily \\
Water-gal
\end{tabular} & \begin{tabular}{|l|} 
Daily Rain \\
inches
\end{tabular} & \begin{tabular}{|l|} 
Daily Rain \\
gallons
\end{tabular} & \begin{tabular}{|l|} 
Tot Accu \\
Water-gal
\end{tabular} & \begin{tabular}{|l|} 
Tot Accu \\
Rain-gal
\end{tabular} \\
\hline $3 / 23 / 95$ & 26.1 & 3601 & 101385 & 0 & 16919 & 0 & 4.5 & 23 & 1130 & & 0 & 44386 & 98569.02 \\
\hline $3 / 24 / 95$ & 13.4 & 832 & 104988 & 3603 & 16919 & 0 & 4.5 & 23 & 834 & & 0 & 45220 & 98569.02 \\
\hline $3 / 25 / 95$ & 13.4 & 832 & 104988 & 0 & 16919 & 0 & 4.5 & 23 & 0 & & 0 & 45220 & 98569.02 \\
\hline $3 / 26 / 95$ & 13.4 & 832 & 104988 & 0 & 16919 & 0 & 4.5 & 23 & 0 & & 0 & 45220 & 98569.02 \\
\hline $3 / 27 / 95$ & 26.4 & 3706 & 104988 & 0 & 16919 & 0 & 4.5 & 23 & 2874 & & 0 & 48094 & 98569.02 \\
\hline $3 / 28 / 95$ & 28.2 & 4368 & 104988 & 0 & 16919 & 0 & 4.5 & 23 & 662 & & 0 & 48756 & 98569.02 \\
\hline $3 / 29 / 95$ & 30.4 & 5304 & 104988 & 0 & 16919 & 0 & 4.5 & 23 & 936 & & 0 & 49692 & 98569.02 \\
\hline $3 / 30 / 95$ & 30.4 & 5304 & 104988 & 0 & 16919 & 0 & 4.5 & 23 & 0 & & 0 & 49692 & 98569.02 \\
\hline $3 / 31 / 95$ & 15.8 & 1195 & 110628 & 5640 & 16919 & 0 & 4.5 & 23 & 1531 & & 0 & 51223 & 98569.02 \\
\hline $4 / 1 / 95$ & 15.8 & 1195 & 110628 & 0 & 16919 & 0 & 4.5 & 23 & 0 & & 0 & 51223 & 98569.02 \\
\hline $4 / 2 / 95$ & 15.8 & 1195 & 110628 & 0 & 16919 & 0 & 4.5 & 23 & 0 & & 0 & 51223 & 98569.02 \\
\hline $4 / 3 / 95$ & 25.7 & 3475 & 110628 & 0 & 16919 & 0 & 4.5 & 23 & 2280 & & 0 & 53503 & 98569.02 \\
\hline $4 / 4 / 95$ & 25.7 & 3475 & 110628 & 0 & 16919 & 0 & 4.5 & 23 & 0 & & 0 & 53503 & 98569.02 \\
\hline $4 / 5 / 95$ & 14.7 & 1025 & 114489 & 3861 & 16919 & 0 & 4.5 & 23 & 1411 & 0.02 & 1629.24 & 54914 & 100198.3 \\
\hline $4 / 6 / 95$ & 19.4 & 1880 & 114489 & 0 & 16919 & 0 & 4.5 & 23 & 855 & & 0 & 55769 & 100198.3 \\
\hline $4 / 7 / 95$ & 15.3 & 1118 & 115780 & 1291 & 16919 & 0 & 4.5 & 23 & 529 & 0.01 & 814.62 & 56298 & 101012.9 \\
\hline $4 / 8 / 95$ & 15.3 & 1118 & 115780 & 0 & 16919 & 0 & 4.5 & 23 & 0 & & 0 & 56298 & 101012.9 \\
\hline $4 / 9 / 95$ & 15.3 & 1118 & 115780 & 0 & 16919 & 0 & 4.5 & 23 & 0 & & 0 & 56298 & 101012.9 \\
\hline $4 / 10 / 95$ & 20.9 & 2200 & 116960 & 1180 & 16919 & 0 & 4.5 & 23 & 2262 & 0.06 & 4887.72 & 58560 & 105900.6 \\
\hline $4 / 11 / 95$ & 23.6 & 2864 & 116960 & 0 & 16919 & 0 & 4.5 & 23 & 664 & & 0 & 59224 & 105900.6 \\
\hline 4,4205 & ic.2 & 518 & 110504 & 2004 & 10010 & 2 & 4.5 & 23 & EEs & 0.30 & 29320.32 & รง70ิ2 & i35220. \\
\hline $4 / 13 / 95$ & 18.6 & 1715 & 119565 & 1 & 16919 & 0 & 4.5 & 23 & 898 & 0.06 & .4887 .72 & 60680 & 140114.6 \\
\hline $4 / 14 / 95$ & 25.1 & 3292 & 119565 & 0 & 16919 & 0 & 4.5 & 23 & 1577 & 0.08 & 6516.96 & 62257 & 146631.6 \\
\hline $4 / 15 / 95$ & 25.1 & 3292 & 119565 & 0 & 16919 & 0 & 4.5 & 23 & 0 & & 0 & 62257 & 146631.6 \\
\hline $4 / 16 / 95$ & 25.1 & 3292 & 119565 & 0 & 16919 & 0 & 4.5 & 23 & 0 & & 0 & 62257 & 146631.6 \\
\hline $4 / 17 / 95$ & 25.1 & 3292 & 119565 & 0 & 16919 & 0 & 4.5 & 23 & 0 & & 0 & 62257 & 146631.6 \\
\hline $4 / 18 / 95$ & 15.4 & 1133 & 125702 & 6137 & 16919 & 0 & 4.6 & 23 & 3978 & & 0 & 66235 & 146631.6 \\
\hline 4/19/95 & 19.3 & 1859 & 125702 & 0 & 16919 & 0 & 4.6 & 23 & 726 & & 0 & 66961 & 146631.6 \\
\hline $4 / 20 / 95$ & 22.7 & 2621 & 125702 & 0 & 16919 & 0 & 4.7 & 24 & 763 & 0.14 & 11404.68 & 67724 & 158036.3 \\
\hline $4 / 21 / 95$ & 25.6 & 3444 & 125702 & 0 & 16919 & 0 & 4.7 & 24 & 823 & & 0 & 68547 & 158036.3 \\
\hline $4 / 22 / 95$ & 25.6 & 3444 & 125702 & 0 & 16919 & 0 & 4.7 & 24 & 0 & & 0 & 68547 & 158036.3 \\
\hline $4 / 23 / 95$ & 25.6 & 3444 & 125702 & o & 16919 & 0 & 4.7 & 24 & 0 & & 0 & 68547 & 158036.3 \\
\hline $4 / 24 / 95$ & 30.9 & 5482 & 125702 & 0 & 16919 & 0 & 4.7 & 24 & 2038 & & 0 & 70585 & 158036.3 \\
\hline $4 / 25 / 95$ & 20.6 & 2135 & 129570 & 3868 & 16919 & 0 & 4.7 & 24 & 521 & & 0 & 71106 & 158036.3 \\
\hline 4/26/95 & 20.6 & 2135 & 129570 & 0 & 16919 & 0 & 4.7 & 24 & 0 & & 0 & 71106 & 158036.3 \\
\hline $4 / 27 / 95$ & 16.9 & 1388 & 131600 & 2030 & 16919 & 0 & 4.7 & 24 & 1283 & 0.34 & 27697.08 & 72389 & 185733.4 \\
\hline $4 / 28 / 95$ & 16.9 & 1388 & 131600 & 0 & 16919 & 0 & 4.7 & 24 & 0 & 0.47 & 38287.14 & 72389 & 224020.5 \\
\hline 4/29/95 & 16.9 & 1388 & 131600 & 0 & 16919 & 0 & 4.7 & 24 & 0 & & 0 & 72389 & 224020.5 \\
\hline $4 / 30 / 95$ & 16.9 & 1388 & 131600 & 0 & 16919 & 0 & 4.7 & 24 & 0 & & 0 & 72389 & 224020.5 \\
\hline
\end{tabular}




\begin{tabular}{|c|c|c|c|c|c|c|c|c|c|c|c|c|c|}
\hline DATE & Pri. Level & Pri. Level & Pri/Sec & Priflow & Sec Flow & Sec Flow & Sec Level & Sec Level & Total Daily & Daily Rain & Daily Rain & Tot Accu & \begin{tabular}{|l|} 
Tot Accu \\
Rain-gal
\end{tabular} \\
\hline & inches & gallons & total flow & & & & & & & & & & \\
\hline $5 / 1 / 95$ & 39 & 10604 & 133570 & 1970 & 16919 & 0 & 4.8 & 24 & 11186 & & 0 & 83575 & 224020.5 \\
\hline $5 / 2 / 95$ & 37.4 & 9264 & 135486 & 1916 & 16919 & 0 & 4.8 & 24 & 576 & & 0 & 84151 & 224020.5 \\
\hline $5 / 3 / 95$ & 19.7 & 1943 & 143531 & 8045 & 16919 & o & 4.8 & 24 & 724 & & 0 & 84875 & 224020.5 \\
\hline $5 / 4 / 95$ & 24.9 & 3233 & 143531 & 0 & 16919 & 0 & 4.8 & 24 & 1290 & & 0 & 86165 & 224020.5 \\
\hline $5 / 5 / 95$ & 28.4 & 4445 & 143531 & 0 & 16919 & 0 & 4.8 & 24 & 1212 & 0.02 & 1629.24 & 87377 & 225649.7 \\
\hline $5 / 6 / 95$ & 28.4 & 4445 & 143531 & 0 & 16919 & 0) & 4.8 & 24 & 0 & & 0 & 87377 & 225649.7 \\
\hline $5 / 7 / 95$ & 28.4 & 4445 & 143531 & 0 & 16919 & 0 & 4.8 & 24 & 0 & 0.05 & 4073.1 & 87377 & 229722.8 \\
\hline $5 / 8 / 95$ & 32.9 & 6550 & 144035 & 504 & 16919 & 0 & 4.9 & 25 & 2610 & 0.01 & 814.62 & 89987 & 230537.5 \\
\hline $5 / 9 / 95$ & 17.5 & 1500 & 149725 & 5690 & 16919 & 0 & 4.9 & 25 & 640 & 0.53 & 43174.86 & 90627 & 273712.3 \\
\hline 5/10/95 & 21.7 & 2379 & 149725 & 0 & 16919 & 0 & 5. & 25 & 879 & 0.02 & 1629.24 & 91506 & 275341.6 \\
\hline $5 / 11 / 95$ & 21.7 & 2379 & 149725 & 0 & 16919 & 0 & 5 & 25 & 0 & 0.1 & 8146.2 & 91506 & 283487.8 \\
\hline $5 / 12 / 95$ & 29.9 & 5041 & 149761 & 36 & 16919 & 0 & 5 & 25 & 2698 & & 0 & 94204 & 283487.8 \\
\hline $5 / 13 / 95$ & 29.9 & 5041 & 149761 & 0 & 16919 & 0 & 5 & 25 & 0 & 0.05 & 4073.1 & 94204 & 287560.9 \\
\hline $5 / 14 / 95$ & 29.9 & 5041 & 149761 & 0 & 16919 & 0 & 5 & 25 & 0 & & 0 & 94204 & 287560.9 \\
\hline $5 / 15 / 95$ & 28.8 & 4599 & 153212 & 3451 & 16919 & 0 & 5.2 & 26 & 3010 & & 0 & 97214 & 287560.9 \\
\hline $5 / 16 / 95$ & 31.3 & 5686 & 153212 & 0 & 16919 & 0 & 5.3 & 27 & 1088 & & 0 & 98302 & 287560.9 \\
\hline $5 / 17 / 95$ & 34.7 & 7560 & 153213 & 1 & 16919 & 0 & 5.4 & 27 & 1875 & & 0 & 100177 & 287560.9 \\
\hline $5 / 18 / 95$ & 34.7 & 7560 & 153213 & 0 & 16919 & 0 & 5.4 & 27 & 0 & & 0 & 100177 & 287560.9 \\
\hline $5 / 19 / 95$ & 35.7 & 8132 & 153213 & 0 & 16919 & 0 & 5.4 & 27 & 572 & & 0 & 100749 & 287560.9 \\
\hline $5 / 20 / 95$ & 35.7 & 8132 & 153213 & 0 & 16919 & 0 & 5.4 & 27 & 0 & & 0 & 100749 & 287560.9 \\
\hline $5 / 21 / 95$ & 35.7 & 8132 & 153213 & 0 & 16919 & uf & 0.4 & $\angle 1$ & vi & & u) & $10074 y$ & $207500 . y$ \\
\hline $5 / 22 / 95$ & 23.2 & 2752 & 160657 & 7444 & 16919 & 0 & 5.7 & 29 & 2066 & & 0 & 102815 & 287560.9 \\
\hline $5 / 23 / 95$ & 25.5 & 3414 & 160657 & 0 & 16919 & 0 & 5.8 & 29 & 662 & & 0 & 103477 & 287560.9 \\
\hline $5 / 24 / 95$ & 27.7 & 4179 & 160733 & 76 & 16919 & 0 & 5.9 & 30 & 842 & & 0 & 104319 & 287560.9 \\
\hline $5 / 25 / 95$ & 16.9 & 1388 & 160733 & 0 & 16919 & 0 & 6 & 30 & -2791 & 0.01 & 814.62 & 101528 & 288375.5 \\
\hline $5 / 26 / 95$ & 11.4 & 575 & 165331 & 4598 & 16919 & 0 & 6.1 & 34 & 3789 & & 0 & 105317 & 288375.5 \\
\hline $5 / 27 / 95$ & 11.4 & 575 & 165331 & 0 & 16919 & 0 & 6.1 & 34 & 0 & & 0 & 105317 & 288375.5 \\
\hline $5 / 28 / 95$ & 11.4 & 575 & 165331 & 0 & 16919 & 0 & 6.1 & 34 & 0 & & 0 & 105317 & 288375.5 \\
\hline $5 / 29 / 95$ & 11.4 & 575 & 165331 & 0 & 16919 & 0 & 6.1 & 34 & 0 & & 0 & 105317 & 288375.5 \\
\hline $5 / 30 / 95$ & 24.6 & 3147 & 165331 & 0 & 16919 & 0 & 6.7 & 57 & 2595 & & 0 & 107912 & 288375.5 \\
\hline $5 / 31 / 95$ & 28.2 & 4368 & 165331 & 0 & 16919 & 0 & 7 & 68 & 1232 & & 0 & 109144 & 288375.5 \\
\hline $6 / 1 / 95$ & 28.2 & 4368 & 165331 & 0 & 16919 & 0 & 7 & 68 & 0 & & 0 & 109144 & 288375.5 \\
\hline $6 / 2 / 95$ & 28.2 & 4368 & 165331 & 0 & 16919 & 0 & 7 & 68 & 0 & & 0 & 109144 & 288375.5 \\
\hline $6 / 3 / 95$ & 28.2 & 4368 & 165331 & 0 & 16919 & 0 & 7 & 68 & 0 & & 0 & 109144 & 288375.5 \\
\hline $6 / 4 / 95$ & 28.2 & 4368 & 165331 & 0 & 16919 & 0 & 7 & 68 & 0 & 0.08 & 6516.96 & 109144 & 294892.4 \\
\hline $6 / 5 / 95$ & 33.3 & 6772 & 165331 & 0 & 16919 & 0 & 8 & 109 & 2445 & & 0 & 111589 & 294892.4 \\
\hline $6 / 6 / 95$ & 27.1 & 3954 & 168330 & 2999 & 16919 & 0 & 8.4 & 127 & 199 & 0.3 & 24438.6 & 111788 & 319331 \\
\hline $6 / 7 / 95$ & 27.1 & 3954 & 168330 & 0 & 16919 & 0 & 8.4 & 127 & 0 & & 0 & 111788 & 319331 \\
\hline $6 / 8 / 95$ & 27.1 & 3954 & 168330 & 0 & 16919 & 0 & 8.4 & 127 & 0 & & 0 & 111788 & 319331 \\
\hline
\end{tabular}


Trench 31

Sheet1

\begin{tabular}{|c|c|c|c|c|c|c|c|c|c|c|c|c|c|}
\hline DATE & Pri. Level & Pri. Level & Pri/Sec & Pri Flow & Sec Flow & Sec Flow & Sec Level & Sec Level & Total Daily & Daily Rain & Daily Rain & Tot Accu & Tol Accu \\
\hline & inches & gallons & total flow & daily & & & inches & gallons & Water-gal & & gallons & Water-gal & Rain-gal \\
\hline $6 / 9 / 95$ & 27.1 & 3954 & 168330 & 0 & 16919 & 0 & 8.4 & $\begin{array}{r}127 \\
\end{array}$ & of & & 0 & 111788 & 319331 \\
\hline $6 / 10 / 95$ & 27.1 & 3954 & 168330 & 0 & 16919 & 0 & 8.4 & 127 & 0 & & 0 & 111788 & 319331 \\
\hline $6 / 11 / 95$ & 27.1 & 3954 & 168330 & 0 & 16919 & 0 & 8.4 & 127 & 0 & t & 0 & 111788 & 319331 \\
\hline $6 / 12 / 95$ & 27.1 & 3954 & 168330 & 0 & 16919 & 0 & 8.4 & 127 & 0 & & 0 & 111788 & 319331 \\
\hline $6 / 13 / 95$ & 19.7 & 1943 & 173349 & 5019 & 16919 & 0 & 10.1 & 212 & 3093 & & 0 & 114881 & 319331 \\
\hline $6 / 14 / 95$ & 21.4 & 2331 & 173349 & 0 & 16919 & 0 & 10.3 & 223 & 399 & 0.1 & 8146.2 & 115280 & 327477.2 \\
\hline $6 / 15 / 95$ & 23 & 2696 & 173349 & 0 & 16919 & 0 & 10.5 & 235 & 377 & 0.01 & 814.62 & 115657 & 328291.9 \\
\hline $6 / 16 / 95$ & 24.4 & 3090 & 173349 & 0 & 16919 & 0 & 10.7 & 247 & 406 & 0.12 & 9775.44 & 116063 & 338067.3 \\
\hline $6 / 17 / 95$ & 24.4 & 3090 & 173349 & 0 & 16919 & 0 & 10.7 & 247 & 0 & 0.04 & 3258.48 & 116063 & 341325.8 \\
\hline $6 / 18 / 95$ & 24.4 & 3090 & 173349 & 0 & 16919 & 0 & 10.7 & 247 & 0 & & 0 & 116063 & 341325.8 \\
\hline $6 / 19 / 95$ & 28 & 4291 & 173349 & 0 & 16919 & 0 & 11.3 & 282 & 1236 & 0.12 & 9775.44 & 117299 & 351101.2 \\
\hline $6 / 20 / 95$ & 28 & 4291 & 173349 & 0 & 16919 & 0 & 11.3 & 282 & 0 & & 0 & 117299 & 351101.2 \\
\hline $6 / 21 / 95$ & 28 & 4291 & 173349 & 0 & 16919 & 0 & 11.3 & 282 & 0 & & 0 & 117299 & 351101.2 \\
\hline $6 / 22 / 95$ & 28 & 4291 & 173349 & 0 & 16919 & 0 & 11.3 & 282 & 0 & & 0 & 117299 & 351101.2 \\
\hline $6 / 23 / 95$ & 28 & 4291 & 173349 & 0 & 16919 & 0 & 11.3 & 282 & 0 & & 0 & 117299 & 351101.2 \\
\hline $6 / 24 / 95$ & 28 & 4291 & 173349 & 0 & 16919 & 0 & 11.3 & 282 & o & & 0 & 117299 & 351101.2 \\
\hline $6 / 25 / 95$ & 28 & 4291 & 173349 & 0 & 16919 & 0 & 11.3 & 282 & 0 & & 0 & 117299 & 351101.2 \\
\hline $6 / 26 / 95$ & 15.7 & 1180 & 178543 & 4922 & 17191 & 272 & 6.4 & 45 & 1846 & & 0 & 119145 & 351101.2 \\
\hline $6 / 27 / 95$ & 17.6 & 1519 & 178543 & 0 & 17191 & 0 & 6.7 & 57 & 351 & & 0 & 119496 & 351101.2 \\
\hline $6 / 28 / 95$ & 19.3 & 1859 & 178543 & 0 & 17191 & 0 & 7 & 68 & 351 & & 0 & 119847 & 351101.2 \\
\hline$b / \angle 9 / 4 b$ & $\angle 1.1$ & $\angle \angle 43$ & 170543 & $\hat{v}$ & iTisi & $\hat{v}$ & 7.3 & $\hat{\mathbf{v} v}$ & S30 & & i & 120243 & 351101.2 \\
\hline $6 / 30 / 95$ & 21.1 & 2243 & 178543 & 0 & 17191 & 0 & 7.3 & 80 & 0 & & 0 & 120243 & 351101.2 \\
\hline $7 / 1 / 95$ & 21.1 & 2243 & 178543 & 0 & 17191 & 0 & 7.3 & 80 & 0 & 0.02 & 1629.24 & 120243 & 352730.5 \\
\hline $7 / 2 / 95$ & 21.1 & 2243 & 178543 & 0 & 17191 & 0 & 7.3 & 80 & 0 & & 0 & 120243 & 352730.5 \\
\hline $7 / 3 / 95$ & 21.1 & 2243 & 178543 & 0 & 17191 & 0 & 7.3 & 80 & 0 & & 0 & 120243 & 352730.5 \\
\hline $7 / 4 / 95$ & 21.1 & 2243 & 178543 & 0 & 17191 & 0 & 7.3 & 80 & 0 & & 0 & 120243 & 352730.5 \\
\hline $7 / 5 / 95$ & 27.8 & 4216 & 178544 & 1 & 17191 & 0 & 9.4 & 175 & 2069 & & 0 & 122312 & 352730.5 \\
\hline $7 / 6 / 95$ & 28.5 & 4484 & 178544 & 0 & 17191 & 0 & 9.7 & 190 & 283 & 0.02 & 1629.24 & 122595 & 354359.7 \\
\hline $7 / 7 / 95$ & 29.3 & 4798 & 178544 & 0 & 17191 & 0 & 10 & 206 & 330 & 0.02 & 1629.24 & 122925 & 355988.9 \\
\hline $7 / 8 / 95$ & 29.3 & 4798 & 178544 & 0 & 17191 & 0 & 10 & 206 & 0 & 0.2 & 16292.4 & 122925 & 372281.3 \\
\hline $7 / 9 / 95$ & 29.3 & 4798 & 178544 & 0 & 17191 & 0 & 10 & 206 & 0 & 0.04 & 3258.48 & 122925 & 375539.8 \\
\hline $7 / 10 / 95$ & 29.3 & 4798 & 178544 & 0 & 17191 & 0 & 10 & 206 & 0 & & 0 & 122925 & 375539.8 \\
\hline $7 / 11 / 95$ & 29.3 & 4798 & 178544 & 0 & 17191 & 0 & 10 & 206 & 0 & & 0 & 122925 & 375539.8 \\
\hline $7 / 12 / 95$ & 29.3 & 4798 & 178544 & 0 & 17191 & 0 & 10 & 206 & 0 & & 0 & 122925 & 375539.8 \\
\hline $7 / 13 / 95$ & 29.3 & 4798 & 178544 & 0 & 17191 & 0 & 10 & 206 & 0 & & 0 & 122925 & 375539.8 \\
\hline $7 / 14 / 95$ & 17.1 & 1425 & 183800 & 5007 & 17440 & 249 & 7.3 & 80 & 1757 & & 0 & 124682 & 375539.8 \\
\hline $7 / 15 / 95$ & 17.1 & 1425 & 183800 & 0 & 17440 & 0 & 7.3 & 80 & 요 & & 0 & 124682 & 375539.8 \\
\hline $7 / 16 / 95$ & 17.1 & 1425 & 183800 & 0 & 17440 & 0 & 7.3 & 80 & 0 & & 0 & 124682 & 375539.8 \\
\hline $7 / 17 / 95$ & 17.1 & 1425 & 183800 & 0 & 17440 & 0 & 7.3 & 80 & 이 & & 0 & 124682 & 375539.8 \\
\hline
\end{tabular}




\begin{tabular}{|c|c|c|c|c|c|c|c|c|c|c|c|c|c|}
\hline DATE & Pri. Level & Pri. Level & Pri/Sec & Pri Flow & Sec Flow & Sec Flow & Sec Level & Sec Level & Total Daily & Daily Rain & Daily Rain & Tot Accu & Tot Accu \\
\hline & inches & gallons & total flow & daily & total & daily & inches & gallons & Water-gal & inches & gallons & Water-gal & Rain-gal \\
\hline $7 / 18 / 95$ & 17.1 & 1425 & 183800 & 0 & 17440 & 0 & 7.3 & 80 & 0 & & 0 & 124682 & 375539.8 \\
\hline $7 / 19 / 95$ & 17.1 & 1425 & 183800 & 0 & 17440 & 0 & 7.3 & 80 & 0 & & 0 & 124682 & 375539.8 \\
\hline $7 / 20 / 95$ & 17.1 & 1425 & 183800 & 0 & 17440 & 0 & 7.3 & 80 & 0 & & 0 & 124682 & 375539.8 \\
\hline $7 / 21 / 95$ & 17.1 & 1425 & 183800 & 0 & 17440 & 0 & 7.3 & 80 & 0 & & 0 & 124682 & 375539.8 \\
\hline $7 / 22 / 95$ & 17.1 & 1425 & 183800 & 0 & 17440 & 0 & 7.3 & 80 & 우 & & 0 & 124682 & 375539.8 \\
\hline 7/23/95 & 17.1 & 1425 & 183800 & 0 & 17440 & 0 & 7.3 & 80 & 0 & & 0 & 124682 & 375539.8 \\
\hline 7/24/95 & 17.1 & 1425 & 183800 & 0 & 17440 & 0 & 7.3 & 80 & 0 & & 0 & 124682 & 375539.8 \\
\hline 7/25/95 & 17.1 & 1425 & 183800 & 0 & 17440 & 0 & 7.3 & 80 & 0 & & 0 & 124682 & 375539.8 \\
\hline $7 / 26 / 95$ & 17.1 & 1425 & 183800 & 0 & 17440 & 0 & 7.3 & 80 & 0 & 0.04 & 3258.48 & 124682 & 378798.3 \\
\hline $7 / 27 / 95$ & 17.1 & 1425 & 183800 & 0 & 17440 & 0 & 7.3 & 80 & 0 & & 0 & 124682 & 378798.3 \\
\hline $7 / 28 / 95$ & 14.4 & 980 & 188100 & 4040 & 17700 & 260 & 8.1 & 114 & 3889 & & 0 & 128571 & 378798.3 \\
\hline $7 / 29 / 95$ & 14.4 & 980 & 188100 & 0 & 17700 & 0 & 8.1 & 114 & 0 & & 0 & 128571 & 378798.3 \\
\hline $7 / 30 / 95$ & 14.4 & 980 & 188100 & 이 & 17700 & 0 & 8.1 & 114 & 0 & & 0 & 128571 & 378798.3 \\
\hline $7 / 31 / 95$ & 14.4 & 980 & 188100 & 0 & 17700 & 0 & 8.1 & 114 & 0 & & 0 & 128571 & 378798.3 \\
\hline $8 / 1 / 95$ & 14.4 & 980 & 188100 & 0 & 17700 & 0 & 8.1 & 114 & 0 & & 0 & 128571 & 378798.3 \\
\hline $8 / 2 / 95$ & 14.4 & 980 & 188100 & 0 & 17700 & 0 & 8.1 & 114 & 0 & & 0 & 128571 & 378798.3 \\
\hline $8 / 3 / 95$ & 14.4 & 980 & 188100 & 0 & 17700 & 0 & 8.1 & 114 & D & & 0 & 128571 & 378798.3 \\
\hline $8 / 4 / 95$ & 14.4 & 980 & 188100 & 0 & 17700 & 0 & 8.1 & 114 & 0 & & 0 & 128571 & 378798.3 \\
\hline $8 / 5 / 95$ & 14.4 & 980 & 188100 & 0 & 17700 & 0 & 8.1 & 114 & 0 & & 0 & 128571 & 378798.3 \\
\hline $8 / 6 / 95$ & 14.4 & 980 & 188100 & 0 & 17700 & 0 & 8.1 & 114 & 0 & & 0 & 128571 & 378798.3 \\
\hline $8 / 7 / 95$ & 14.4 & 980 & 188700 & 0 & $1 / 100$ & 0 & 8.1 & 174 & u) & & 0 & 128571 & 378798.3 \\
\hline $8 / 8 / 95$ & 14.4 & 980 & 188100 & 0 & 17700 & 0 & 8.1 & 114 & 0 & & 0 & 128571 & 378798.3 \\
\hline $8 / 9 / 95$ & 14.4 & 980 & 188100 & - & 17700 & 0 & 8.1 & 114 & 0 & & 0 & 128571 & 378798.3 \\
\hline $8 / 10 / 95$ & 14.4 & 980 & 188100 & 0 & 17700 & 0 & 8.1 & 114 & 0 & 0.06 & 4887.72 & 128571 & 383686 \\
\hline $8 / 11 / 95$ & 14.4 & 980 & 188100 & 0 & 17700 & 0 & 8.1 & 114 & 0 & & 0 & 128571 & 383686 \\
\hline $8 / 12 / 95$ & 14.4 & 980 & 188100 & 0 & 17700 & 0 & 8.1 & 114 & 0 & & 0 & 128571 & 383686 \\
\hline $8 / 13 / 95$ & 14.4 & 980 & 188100 & 0 & 17700 & 0 & 8.1 & 114 & 0 & & 0 & 128571 & 383686 \\
\hline $8 / 14 / 95$ & 14.4 & 980 & 188100 & 0 & 17700 & 0 & 8.1 & 114 & 0 & & 0 & 128571 & 383686 \\
\hline $8 / 15 / 95$ & 14.4 & 980 & 188100 & o) & 17700 & 0 & 8.1 & 114 & 0 & 0.01 & 814.62 & 128571 & 384500.6 \\
\hline $8 / 16 / 95$ & 14.4 & 980 & 188100 & 0 & 17700 & 0 & 8.1 & 114 & 0 & & 0 & 128571 & 384500.6 \\
\hline $8 / 17 / 95$ & 14.4 & 980 & 188100 & of & 17700 & 0 & 8.1 & 114 & 0 & & 0 & 128571 & 384500.6 \\
\hline $8 / 18 / 95$ & 14.4 & 980 & 188100 & 0 & 17700 & 0 & 8.1 & 114 & 0 & & 0 & 128571 & 384500.6 \\
\hline $8 / 19 / 95$ & 14.4 & 980 & 188100 & 0 & 17700 & 0 & 8.1 & 114 & 0 & & 0 & 128571 & 384500.6 \\
\hline $8 / 20 / 95$ & 14.4 & 980 & 188100 & 0 & 17700 & 0 & 8.1 & 114 & 0 & & 0 & 128571 & 384500.6 \\
\hline $8 / 21 / 95$ & 14.4 & 980 & 188100 & 0 & 17700 & 0 & 8.1 & 114 & o & & 0 & 128571 & 384500.6 \\
\hline $8 / 22 / 95$ & 14.4 & 980 & 188100 & 0 & 17700 & 0 & 8.1 & 114 & 0 & & 0 & 128571 & 384500.6 \\
\hline $8 / 23 / 95$ & 14.4 & 980 & 188100 & 0 & 17700 & 0 & 8.1 & 114 & 0 & & 0 & 128571 & 384500.6 \\
\hline $8 / 24 / 95$ & 14.4 & 980 & 188100 & 0 & 17700 & 0 & 8.1 & 114 & 0 & & ㅇ. & 128571 & 384500.6 \\
\hline $8 / 25 / 95$ & 14.4 & 980 & 188100 & 0 & 17700 & 이 & 8.11 & 114 & 이 & & 의 & 128571 & 384500.6 \\
\hline
\end{tabular}




\begin{tabular}{|c|c|c|c|c|c|c|c|c|c|c|c|c|c|}
\hline DATE & Pri. Level & Pri. Level & Pri/Sec . & Pri Flow & Sec Flow & Sec Flow & Sec Level & Sec Level & Total Daily & Daily Rain & Daily Rain & Tot Accu & Tot Accu \\
\hline & & & & & & & & & & inches & & & Rain-gal \\
\hline $8 / 26 / 95$ & \begin{tabular}{|r|}
14.4 \\
\end{tabular} & 980 & 188100 & 0 & 17700 & 0 & 8.1 & 114 & 0 & & 0 & 128571 & 384500.6 \\
\hline $8 / 27 / 95$ & 14.4 & 980 & 188100 & 0 & 17700 & 0 & 8.1 & 114 & 0 & & 0 & 128571 & 384500.6 \\
\hline $8 / 28 / 95$ & 14.4 & 980 & 188100 & 0 & 17700 & 0 & 8.1 & 114 & 0 & & 0 & 128571 & 384500.6 \\
\hline $8 / 29 / 95$ & 14.4 & 980 & 188100 & 0 & 17700 & 0 & 8.1 & 114 & 0 & & 0 & 128571 & 384500.6 \\
\hline $8 / 30 / 95$ & 14.4 & 980 & 188100 & 0 & 17700 & 0 & 8.1 & 114 & 0 & & 0 & 128571 & 384500.6 \\
\hline $8 / 31 / 95$ & 14.4 & 980 & 188100 & 0 & 17700 & 0 & 8.1 & 114 & 0 & & 0 & 128571 & 384500.6 \\
\hline $9 / 1 / 95$ & 14.4 & 980 & 188100 & 이 & 17700 & 0 & 8.1 & 114 & 0 & & 0 & 128571 & 384500.6 \\
\hline $9 / 2 / 95$ & 14.4 & 980 & 188100 & 0 & 17700 & 0 & 8.1 & 114 & 0 & & 0 & 128571 & 384500.6 \\
\hline $9 / 3 / 95$ & 14.4 & 980 & 188100 & 이 & 17700 & 0 & 8.1 & 114 & 0 & & 0 & 128571 & 384500.6 \\
\hline 9/4/95 & 14.4 & 980 & 188100 & 0 & 17700 & 0 & 8.1 & 114 & 0 & & 0 & 128571 & 384500.6 \\
\hline $9 / 5 / 95$ & 14.4 & 980 & 188100 & 0 & 17700 & 0 & 8.1 & 114 & 0 & & 0 & 128571 & 384500.6 \\
\hline $9 / 6 / 95$ & 14.4 & 980 & 188100 & 0 & 17700 & 0 & 8.1 & 114 & 0 & 0.48 & 39101.76 & 128571 & 423602.4 \\
\hline $9 / 7 / 95$ & 14.4 & 980 & 188100 & 0 & 17700 & 0 & 8.1 & 114 & 0 & 0.19 & 15477.78 & 128571 & 439080.2 \\
\hline $9 / 8 / 95$ & 14.4 & 980 & 188100 & 0 & 17700 & 0 & 8.1 & 114 & 0 & & 0 & 128571 & 439080.2 \\
\hline 9/9/95 & 14.4 & 980 & 188100 & 0 & 17700 & 0 & 8.1 & 114 & 0 & & 0 & 128571 & 439080.2 \\
\hline 9/10/95 & 14.4 & 980 & 188100 & 0 & 17700 & 0 & 8.1 & 114 & 0 & & 0 & 128571 & 439080.2 \\
\hline $9 / 11 / 95$ & 14.4 & 980 & 188100 & 0 & 17700 & 0 & 8.1 & 114 & 0 & & 0 & 128571 & 439080.2 \\
\hline $9 / 12 / 95$ & 14.4 & 980 & 188100 & 0 & 17700 & 0 & 8.1 & 114 & 0 & & 0 & 128571 & 439080.2 \\
\hline 9/13/95 & 14.4 & 980 & 188100 & 0 & 17700 & 0 & 8.1 & 114 & 0 & & 0 & 128571 & 439080.2 \\
\hline $9 / 14 / 95$ & 14.4 & 980 & 188100 & 0. & 17700 & 0 & 8.1 & 114 & 0 & & 0 & 128571 & 439080.2 \\
\hline 9/15/95 & 14.4 & 980 & 188100 & 0 & $1 / 100$ & 0 & 8.7 & 114 & $\mathbf{u}$ & & v & $1285 / 1$ & 439080.2 \\
\hline $9 / 16 / 95$ & 14.4 & 980 & 188100 & 0 & 17700 & 0 & 8.1 & 114 & 0 & & 0 & 128571 & 439080.2 \\
\hline $9 / 17 / 95$ & 14.4 & 980 & 188100 & 0 & 17700 & 0 & 8.1 & 114 & 0 & & 0 & 128571 & 439080.2 \\
\hline $9 / 18 / 95$ & 14.4 & 980 & 188100 & 0 & 17700 & 0 & 8.1 & 114 & 0 & & 0 & 128571 & 439080.2 \\
\hline 9/19/95 & 14.4 & 980 & 188100 & 0 & 17700 & 0 & 8.1 & 114 & 0 & & o & 128571 & 439080.2 \\
\hline $9 / 20 / 95$ & 14.4 & 980 & 188100 & 0 & 17700 & 0 & 8.1 & 114 & 0 & & 0 & 128571 & 439080.2 \\
\hline $9 / 21 / 95$ & 14.4 & 980 & 188100 & 0 & 17700 & 0 & 8.1 & 114 & 0 & & 0 & 128571 & 439080.2 \\
\hline $9 / 22 / 95$ & 14.4 & 980 & 188100 & 0 & 17700 & 0 & 8.1 & 114 & 0 & & 0 & 128571 & 439080.2 \\
\hline $9 / 23 / 95$ & 14.4 & 980 & 188100 & 0 & 17700 & 0 & 8.1 & 114 & 0 & & 0 & 128571 & 439080.2 \\
\hline $9 / 24 / 95$ & 14.4 & 980 & 188100 & 0 & 17700 & 0 & 8.1 & 114 & 0 & & 0 & 128571 & 439080.2 \\
\hline $9 / 25 / 95$ & 14.4 & 980 & 188100 & 0 & 17700 & 0 & 8.1 & 114 & 0 & & 0 & 128571 & 439080.2 \\
\hline $9 / 26 / 95$ & 14.4 & 980 & 188100 & 0 & 17700 & 0 & 8.1 & 114 & 0 & & 0 & 128571 & 439080.2 \\
\hline $9 / 27 / 95$ & 14.4 & 980 & 188100 & 이 & 17700 & 0 & 8.1 & 114 & 0 & 0.1 & 8146.2 & 128571 & 447226.4 \\
\hline $9 / 28 / 95$ & 14.4 & 980 & 188100 & ㅇ․ & 17700 & 0 & 8.1 & 114 & 0 & & 0 & 128571 & 447226.4 \\
\hline $9 / 29 / 95$ & 14.4 & 980 & 188100 & 0 & 17700 & 0 & 8.1 & 114 & 0 & & 0 & 128571 & 447226.4 \\
\hline $9 / 30 / 95$ & 14.4 & 980 & 188100 & 0 & 17700 & 0 & 8.1 & 114 & 0 & 0.02 & 1629.24 & 128571 & 448855.6 \\
\hline $10 / 1 / 95$ & 14.4 & 980 & 188100 & 0 & 17700 & 0 & 8.1 & 114 & 0 & & 이 & 128571 & 448855.6 \\
\hline $10 / 2 / 95$ & 14.4 & 980 & 188100 & 0 & 17700 & 0 & 8.1 & 114 & 0 & 0.09 & 7331.58 & 128571 & 456187.2 \\
\hline $10 / 3 / 95$ & 27.3 & 4029 & 193128 & 4438 & 18290 & 590 & 21 & 1146 & 9109 & 0.38 & 30955.56 & 137680 & 487142.8 \\
\hline
\end{tabular}


Trench 31

Sheet1

\begin{tabular}{|c|c|c|c|c|c|c|c|c|c|c|c|c|c|}
\hline DATE & Pri. Level & Pri. Level & Pri/Sec & Pri Flow & Sec Flow & Sec Flow & Sec Level & Sec Level & Total Daily & Daily Rain & Daily Rain & Tol Accu & Tot Accu \\
\hline & inches & gallons & total flow & daily & total & daily & inches & gallons & Water-gal & inches & gallons & Water-gal & Rain-gal \\
\hline $10 / 4 / 95$ & 27.3 & 4029 & 193128 & 0 & 18290 & 0 & 21 & 1146 & 0 & & 0 & 137680 & 487142.8 \\
\hline $10 / 5 / 95$ & 27.3 & 4029 & 193129 & 1 & 18290 & 0 & 21.1 & 1157 & 12 & & 0 & 137692 & 487142.8 \\
\hline $10 / 6 / 95$ & 27.4 & 4066 & 194129 & 0 & 19290 & 1000 & 7.1 & 72 & -48 & & 0 & 137644 & 487142.8 \\
\hline $10 / 7 / 95$ & 27.4 & 4066 & 194129 & 0 & 19290 & 0 & 7.1 & 72 & 0 & & 0 & 137644 & 487142.8 \\
\hline 10/8/95 & 27.4 & 4066 & 194129 & 0 & 19290 & 0 & 7.1 & 72 & 0 & & 0 & 137644 & 487142.8 \\
\hline $10 / 9 / 95$ & 9.2 & 344 & 197775 & 3646 & 19290 & 0 & 8.4 & 127 & -21 & & 0 & 137623 & $4 8 7 \longdiv { 1 4 2 . 8 }$ \\
\hline $10 / 10 / 95$ & 9.7 & 395 & 197775 & 0 & 19290 & 0 & 8.7 & 141 & 65 & 0.11 & 8960.82 & 137688 & 496103.6 \\
\hline $10 / 11 / 95$ & 10 & 426 & 197777 & 2 & 19290 & 0 & 8.8 & 145 & 37 & 0.18 & 14663.16 & 137725 & 510766.7 \\
\hline $10 / 12 / 95$ & 10.3 & 457 & 197777 & 0 & 19290 & 0 & 9 & 154 & 40 & & 0 & 137765 & 510766.7 \\
\hline $10 / 13 / 95$ & 10.5 & 479 & 197777 & 0 & 19290 & 0 & 9.1 & 159 & 27 & & 0 & 137792 & 510766.7 \\
\hline $10 / 14 / 95$ & 10.5 & 479 & 197777 & 0 & 19290 & 0 & 9.1 & 159 & 0 & & 0 & 137792 & 510766.7 \\
\hline $10 / 15 / 95$ & 10.5 & 479 & 197777 & 0 & 19290 & $\overline{0}$ & 9.1 & 159 & 0 & & 0 & 137792 & 510766.7 \\
\hline $10 / 16 / 95$ & 11.2 & 553 & 197777 & 0 & 19290 & 0 & 9.6 & 185 & 100 & & 0 & 137892 & 510766.7 \\
\hline $10 / 17 / 95$ & 11.2 & 553 & 197777 & 0 & 19290 & 0 & 9.5 & 180 & -5 & 0.09 & 7331.58 & 137887 & 518098.3 \\
\hline $10 / 18 / 95$ & 11.3 & 564 & 197777 & 0 & 19290 & 0 & 9.6 & 185 & 16 & & 0 & 137903 & 518098.3 \\
\hline $10 / 19 / 95$ & 11.4 & 575 & 197777 & 0 & 19290 & 0 & 9.7 & 190 & 16 & & 0 & 137919 & 518098.3 \\
\hline $10 / 20 / 95$ & 11.6 & 597 & 197777 & 0 & 19290 & 0 & 9.7 & 190 & 22 & & 0 & 137941 & 518098.3 \\
\hline $10 / 21 / 95$ & 11.6 & 597 & 197777 & 0 & 19290 & 0 & 9.7 & 190 & 0 & & 0 & 137941 & 518098.3 \\
\hline $10 / 22 / 95$ & 11.6 & 597 & 197777 & 0 & 19290 & 0 & 9.7 & 190 & 0 & & 0 & 137941 & 518098.3 \\
\hline $10 / 23 / 95$ & 11.9 & 630 & 197777 & 0 & 19290 & 0 & 9.8 & 196 & 39 & & 0 & 137980 & 518098.3 \\
\hline iũizuigo & $i 2$ & $04 i$ & 19717 & ù & $19<90$ & $\underline{v}$ & y.o & Iyo & 111 & & u & $13 / 941$ & 318098.3 \\
\hline $10 / 25 / 95$ & 12.1 & 654 & 197777 & 0 & 19290 & 0 & 9.9 & 201 & 18 & 0.02 & 1629.24 & 138009 & 519727.6 \\
\hline $10 / 26 / 95$ & 12.2 & 668 & 197777 & 0 & 19290 & 0 & 9.9 & 201 & 14 & & 0 & 138023 & 519727.6 \\
\hline $10 / 27 / 95$ & 12.2 & 668 & 197777 & 0 & 19290 & 0 & 9.9 & 201 & 0 & & 0 & 138023 & 519727.6 \\
\hline $10 / 28 / 95$ & 12.2 & 668 & 197777 & 0 & 19290 & 0 & 9.9 & 201 & 0 & & 0 & 138023 & 519727.6 \\
\hline $10 / 29 / 95$ & 12.2 & 668 & 197777 & 0 & 19290 & 0 & 9.9 & 201 & 0 & & 0 & 138023 & 519727.6 \\
\hline $10 / 30 / 95$ & 12.5 & 708 & 197777 & 0 & 19290 & 0 & 10 & 206 & 45 & & 0 & 138068 & 519727.6 \\
\hline $10 / 31 / 95$ & 12.5 & 708 & 197777 & 0 & 19290 & 0 & 10 & 206 & 0 & & 0 & 138068 & 519727.6 \\
\hline $11 / 1 / 95$ & 12.5 & 708 & 197777 & 0 & 19290 & 0 & 10.1 & 212 & 6 & & 0 & 138074 & 519727.6 \\
\hline $11 / 2 / 95$ & 12.5 & 708 & 197777 & 0 & 19290 & 0 & 10.1 & 212 & 0 & & 0 & 138074 & 519727.6 \\
\hline $11 / 3 / 95$ & 12.6 & 721 & 197777 & 0 & 19290 & 0 & 10.1 & 212 & 13 & & 0 & 138087 & 519727.6 \\
\hline $11 / 4 / 95$ & 12.6 & 721 & 197777 & 0 & 19290 & 0 & 10.1 & 212 & 0 & & 0 & 138087 & 519727.6 \\
\hline $11 / 5 / 95$ & 12.6 & 721 & 197777 & 0 & 19290 & 0 & 10.1 & 212 & 0 & & 0 & 138087 & 519727.6 \\
\hline $11 / 6 / 95$ & 12.8 & 747 & 197777 & 0 & 19290 & 0 & 10.1 & 212 & 26 & 0.06 & 4887.72 & 138113 & 524615.3 \\
\hline $11 / 7 / 95$ & 12.8 & 747 & 197777 & 0 & 19290 & 0 & 10.1 & 212 & 0 & 0.18 & 14663.16 & 138113 & 539278.4 \\
\hline $11 / 8 / 95$ & 13 & 774 & 197777 & 0 & 19290 & 0 & 10.2 & 218 & 33 & & 0 & 138146 & 539278.4 \\
\hline $11 / 9 / 95$ & 12.9 & 761 & 197777 & 0 & 19290 & ㅇ & 10.2 & 218 & -13 & & 0 & 138133 & 539278.4 \\
\hline $11 / 10 / 95$ & 12.8 & 747 & 197777 & 으 & 19290 & 0 & 10.1 & 212 & -20 & 0.15 & 12219.3 & 138113 & 551497.7 \\
\hline $11 / 11 / 95$ & 12.8 & 747 & 197777 & 이 & 19290 & 0 & 10.1 & 212 & 0 & 0.05 & 4073.1 & 138113 & 555570.8 \\
\hline
\end{tabular}




\begin{tabular}{|c|c|c|c|c|c|c|c|c|c|c|c|c|c|}
\hline DATE & Pri. Level & Pri. Level & Pri/Sec & Pri Flow & Sec Flow & Sec Flow & Sec Level & Sec Level & Total Daily & Daily Rain & Daily Rain & Tot Accu & Tot Accu \\
\hline & inches & gallons & total flow & daily & total & daily & inches & gallons & Water-gal & inches & gallons & Water-gal & Rain-gal \\
\hline $11 / 12 / 95$ & 12.8 & 747 & 197777 & 0 & 19290 & 0 & 10.1 & 212 & 0 & 0.05 & $\begin{array}{l}4073.1 \\
\end{array}$ & 138113 & 559643.9 \\
\hline $11 / 13 / 95$ & 12.9 & 761 & 197777 & 0 & 19290 & 0 & 10.1 & 212 & 14 & 0.15 & 12219.3 & 138127 & 571863.2 \\
\hline $11 / 14 / 95$ & 13.7 & 876 & 197777 & 0 & 19290 & 0 & 10.1 & 212 & 115 & & 0 & 138242 & 571863.2 \\
\hline $11 / 15 / 95$ & 14.1 & 934 & 197777 & 0 & 19290 & 0 & 10.1 & 212 & 58 & 0.01 & 814.62 & 138300 & 572677.9 \\
\hline $11 / 16 / 95$ & 14.3 & 965 & 197777 & 0 & 19290 & 0 & 10.1 & 212 & 31 & & 0 & 138331 & 572677.9 \\
\hline $11 / 17 / 95$ & 14.4 & 980 & 197777 & 0 & 19290 & 0 & 10.1 & 212 & 15 & 0.02 & 1629.24 & 138346 & 574307.1 \\
\hline $11 / 18 / 95$ & 14.4 & 980 & 197777 & 0 & 19290 & 0 & 10.1 & 212 & 0 & & 0 & 138346 & 574307.1 \\
\hline $11 / 19 / 95$ & 14.4 & 980 & 197777 & 0 & 19290 & 0 & 10.1 & 212 & 0 & & 0 & 138346 & 574307.1 \\
\hline $11 / 20 / 95$ & 14.8 & 1041 & 197777 & 0 & 19290 & 0 & 10.1 & 212 & 61 & & 0 & 138407 & 574307.1 \\
\hline $11 / 21 / 95$ & 14.9 & 1056 & 197777 & 0 & 19290 & 0 & 10.1 & 212 & 15 & 0.01 & 814.62 & 138422 & 575121.7 \\
\hline $11 / 22 / 95$ & 14.9 & 1056 & 197777 & 0 & 19290 & 0 & 10.1 & 212 & 0 & & 0 & 138422 & 575121.7 \\
\hline $11 / 23 / 95$ & 14.9 & 1056 & 197777 & 0 & 19290 & 0 & 10.1 & 212 & 0 & 0.16 & 13033.92 & 138422 & 588155.6 \\
\hline $11 / 24 / 95$ & 14.9 & 1056 & 197777 & 0 & 19290 & 0 & 10.1 & 212 & 0 & 0.01 & 814.62 & 138422 & 588970.3 \\
\hline $11 / 25 / 95$ & 14.9 & 1056 & 197777 & 0 & 19290 & 0 & 10.1 & 212 & 0 & & 0 & 138422 & 588970.3 \\
\hline $11 / 26 / 95$ & 14.9 & 1056 & 197777 & 0 & 19290 & 0 & 10.1 & 212 & 0 & & 0 & 138422 & 588970.3 \\
\hline $11 / 27 / 95$ & 15.3 & 1118 & 197777 & 0 & 19290 & 0 & 10.1 & 212 & 62 & 0.19 & 15477.78 & 138484 & 604448 \\
\hline $11 / 28 / 95$ & 15.7 & 1180 & 197777 & 0 & 19290 & 0 & 10.2 & 218 & 68 & & 0 & 138552 & 604448 \\
\hline $11 / 29 / 95$ & 16.1 & 1244 & 197777 & 0 & 19290 & 0 & 10.1 & 212 & 58 & & 0 & 138610 & 604448 \\
\hline $11 / 30 / 95$ & 16.5 & 1316 & 197777 & 0 & 19290 & 0 & 10.2 & 218 & 78 & & 0 & 138688 & 604448 \\
\hline $12 / 1 / 95$ & 16.7 & 1352 & 197777 & 0 & 19290 & 0 & 10.1 & 212 & 30 & & 0 & 138718 & 604448 \\
\hline $1 \angle \angle Y Y 5$ & 10.1 & 1.302 & $|y / 1 \||$, & $u$ & $1 Y \angle Y O$ & $u$ & 10.7 & 212 & $\mathbf{u}$ & & of & 138718 & 604448 \\
\hline $12 / 3 / 95$ & 16.7 & 1352 & 197777 & 0 & 19290 & 0 & 10.1 & 212 & 0 & 0.19 & 15477.78 & 138718 & 619925.8 \\
\hline $12 / 4 / 95$ & 8.1 & 234 & 199141 & 1222 & 19432 & 142 & 6.7 & 57 & 91 & & 0 & 138809 & 619925.8 \\
\hline $12 / 5 / 95$ & 8.7 & 304 & 199142 & 1 & 19432 & 0 & 6.6 & 53 & 67 & & 0 & 138876 & 619925.8 \\
\hline $12 / 6 / 95$ & 9 & 324 & 199142 & 0 & 19432 & 0 & 6.6 & 53 & 20 & & 0 & 138896 & 619925.8 \\
\hline $12 / 7 / 95$ & 9.3 & 355 & 199142 & 0 & 19432 & 0 & 6.6 & 53 & 31 & & 0 & 138927 & 619925.8 \\
\hline $12 / 8 / 95$ & 9.5 & 375 & 199142 & 0 & 19432 & 0 & 6.6 & 53 & 20 & & 0 & 138947 & 619925.8 \\
\hline 12/9/95 & 9.5 & 375 & 199142 & 0 & 19432 & 0 & 6.6 & 53 & 0 & 0.13 & 10590.06 & 138947 & 630515.9 \\
\hline $12 / 10 / 95$ & 9.5 & 375 & 199142 & 0 & 19432 & 0 & 6.6 & 53 & 0 & 0.38 & 30955.56 & 138947 & 661471.4 \\
\hline $12 / 11 / 95$ & 10.1 & 437 & 199142 & 0 & 19432 & 0 & 6.6 & 53 & 62 & 0.4 & 32584.8 & 139009 & 694056.2 \\
\hline $12 / 12 / 95$ & 13.5 & 847 & 199142 & 0 & 19432 & 0 & 6.7 & 57 & 414 & 0.65 & 52950.3 & 139423 & 747006.5 \\
\hline $12 / 13 / 95$ & 27.2 & 3991 & 199142 & 0 & 19432 & ㅇ. & 6.6 & 53 & 3140 & & 0 & 142563 & 747006.5 \\
\hline $12 / 14 / 95$ & 16.8 & 1370 & 202982 & 3840 & 19432 & 0 & 6.6 & 53 & 1219 & 0.02 & 1629.24 & 143782 & 748635.8 \\
\hline $12 / 15 / 95$ & 14.5 & 995 & 204402 & 1420 & 19432 & 0 & 6.6 & 53 & 1045 & & 0 & 144827 & 748635.8 \\
\hline $12 / 16 / 95$ & 14.5 & 995 & 204402 & 0 & 19432 & 0 & 6.6 & 53. & 0 & & 0 & 144827 & 748635.8 \\
\hline $12 / 17 / 95$ & 14.5 & 995 & 204402 & 0 & 19432 & 0 & 6.6. & 53 & 0 & & 0 & 144827 & 748635.8 \\
\hline $12 / 18 / 95$ & 23.8 & 2920 & 204402 & 0 & 19432 & 0 & 6.5 & 49 & 1921 & & 0 & 146748 & 748635.8 \\
\hline $12 / 19 / 95$ & 11.4 & 575 & 207130 & 2728 & 19432 & 0 & 6.5 & 49 & 383 & 0.01 & 814.62 & 147131 & 749450.4 \\
\hline $12 / 20 / 95$ & 15.2 & 1102 & 207130 & 0 & 19432 & 0 & 6.5 & 49 & 527 & & 0 & 147658 & 749450.4 \\
\hline
\end{tabular}




\begin{tabular}{|c|c|c|c|c|c|c|c|c|c|c|c|c|c|}
\hline DATE & Pri. Level & Pri. Level & Pri/Sec & Pri Flow & Sec Flow & Sec Flow & Sec Level & Sec Level & Total Daily & Daily Rain & Daily Rain & Tot Accu & \begin{tabular}{|l} 
Tot Accu \\
Rain-oal
\end{tabular} \\
\hline & inches & gallons & total flow & daily & total & daily & inches & & & & & Waler-gal & Rain-gal \\
\hline $12 / 21 / 95$ & 15.2 & 1102 & 207130 & 0 & 19432 & 0 & 6.5 & 49 & 0 & & 0 & 147658 & 749450.4 \\
\hline $12 / 22 / 95$ & 15.2 & 1102 & 207130 & 0 & 19432 & 0 & 6.5 & 49 & 0 & & 0 & 147658 & 749450.4 \\
\hline $12 / 23 / 95$ & 15.2 & 1102 & 207130 & 0 & 19432 & 0 & 6.5 & 49 & 0 & & 0 & 147658 & 749450.4 \\
\hline $12 / 24 / 95$ & 15.2 & 1102 & 207130 & 0 & 19432 & 0 & 6.5 & 49 & 0 & & 0 & 147658 & 749450.4 \\
\hline $12 / 25 / 95$ & 15.2 & 1102 & 207130 & 0 & 19432 & 0 & 6.5 & 49 & 0 & & 0 & 147658 & 749450.4 \\
\hline $12 / 26 / 95$ & 15.2 & 1102 & 207130 & 0 & 19432 & 0 & 6.5 & 49 & 0 & & 0 & 147658 & 749450.4 \\
\hline $12 / 27 / 95$ & 28.5 & 4484 & 207130 & 0 & 19432 & 0 & 6.4 & 45 & 3378 & & 0 & 151036 & 749450.4 \\
\hline $12 / 28 / 95$ & 12 & 641 & 211272 & 4142 & 19432 & 0 & 6.4 & 45 & 299 & 0.04 & 3258.48 & 151335 & 752708.9 \\
\hline $12 / 29 / 95$ & 15.3 & 1118 & 211272 & 0 & 19432 & 0 & 6.4 & 45 & 477 & 0.22 & 17921.64 & 151812 & 770630.5 \\
\hline $12 / 30 / 95$ & 15.3 & 1118 & 211272 & 0 & 19432 & 0 & 6.4 & 45 & 0 & 0.28 & 22809.36 & 151812 & 793439.9 \\
\hline $12 / 31 / 95$ & 15.3 & 1118 & 211272 & 0 & 19432 & 0 & 6.4 & 45 & 0 & & 0 & 151812 & 793439.9 \\
\hline $1 / 1 / 96$ & 15.3 & 1118 & 211272 & 0 & 19432 & 0 & 6.4 & 45 & 0 & 0.02 & 1629.24 & 151812 & 795069.1 \\
\hline $1 / 2 / 96$ & 35.9 & 7673 & 211272 & -1 & 19433 & 1 & 6.2 & 38 & 6548 & & 0 & 158360 & 795069.1 \\
\hline $1 / 3 / 96$ & 21.4 & 2311 & 218990 & 7718 & 19433 & 0 & 6.4 & 45 & 2363 & & 0 & 160723 & 795069.1 \\
\hline $1 / 4 / 96$ & 26.3 & 3657 & 218990 & 0 & 19433 & 0 & 6.3 & 41 & 1342 & & 0 & 162065 & 795069.1 \\
\hline $1 / 5 / 96$ & 28.7 & 4310 & 218990 & 0 & 19433 & 0 & 6.2 & 38 & 650 & 0.15 & 12219.3 & 162715 & 807288.4 \\
\hline $1 / 6 / 96$ & 28.7 & 4310 & 218990 & 0 & 19433 & 0 & 6.2 & 38 & 0 & 0.01 & 814.62 & 162715 & 808103 \\
\hline $1 / 7 / 96$ & 28.7 & 4310 & 218990 & 0 & 19433 & 0 & 6.2 & 38 & 0 & 0.1 & 8146.2 & 162715 & 816249.2 \\
\hline $1 / 8 / 96$ & 27.4 & 3930 & 223424 & 4434 & 19433 & 0 & 6.2 & 38 & 4054 & & 0 & 166769 & 816249.2 \\
\hline 1/9/96 & 31.2 & 5615 & 223424 & 0 & 19433 & 0 & 6.2 & 38 & 1685 & 0.04 & 3258.48 & 168454 & 819507.7 \\
\hline $1 / 10 / 40$ & 20.1 & $3<01$ & $\angle \angle T i \mid \overrightarrow{0}$ & sivint & $i \overline{9433}$ & $\hat{v}_{1}^{+}$ & $\hat{0.2}$ & 30 & 1370 & & $\boldsymbol{v}_{1}$ & 100024 & 013507.7 \\
\hline $1 / 11 / 96$ & 15.6 & 1080 & 230890 & 3762 & 19433 & 0 & 6.1 & 34 & 1557 & & 0 & 171381 & 819507.7 \\
\hline $1 / 12 / 96$ & 18.3 & 1600 & 231760 & 870 & 19433 & 0 & 6.2 & 38 & 1394 & & 0 & 172775 & 819507.7 \\
\hline $1 / 13 / 96$ & 18.3 & 1600 & 231760 & 0 & 19433 & 0 & 6.1 & 34 & -4 & & 0 & 172771 & 819507.7 \\
\hline $1 / 14 / 96$ & 18.3 & 1600 & 231760 & 0 & 19433 & 0 & 6.1 & 34 & 0 & 0.02 & 1629.24 & 172771 & 821137 \\
\hline $1 / 15 / 96$ & 26.7 & 3627 & 233502 & 1742 & 19433 & 0 & 6.1 & 34 & 3769 & 0.02 & 1629.24 & 176540 & 822766.2 \\
\hline $1 / 16 / 96$ & 28.5 & 4479 & 233690 & 188 & 19433 & 0 & 6.1 & 34 & 1040 & & 0 & 177580 & 822766.2 \\
\hline $1 / 17 / 96$ & 17.4 & 1478 & 237663 & 3973 & 19433 & 0 & 6.1 & 34 & 972 & & 0 & $17 \overline{8552}$ & 822766.2 \\
\hline $1 / 18 / 96$ & 13 & 774 & 239300 & 1637 & 19433 & 0 & 6 & 30 & 929 & 0.28 & 22809.36 & 179481 & 845575.6 \\
\hline $1 / 19 / 96$ & 7 & 134 & 240900 & 1600 & 19433 & 0 & 6.1 & 34 & 964 & 0.01 & 814.62 & 180445 & 846390.2 \\
\hline $1 / 20 / 96$ & 7 & 134 & 240900 & 0 & 19433 & 0 & 6 & 30 & -4 & 0.25 & 20365.5 & 180441 & 866755.7 \\
\hline $1 / 21 / 96$ & 7 & 134 & 240900 & 0 & 19433 & 0 & 6 & 30 & 0 & & 0 & 180441 & 866755.7 \\
\hline $1 / 22 / 96$ & 22.3 & 2491 & 241031 & 131 & 19433 & 0 & 6 & 30 & 2488 & & 0 & 182929 & 866755.7 \\
\hline $1 / 23 / 96$ & 12.9 & 761 & 243592 & 2561 & 19433 & 0 & 6 & 30 & 831 & 0.01 & 814.62 & 183760 & 867570.3 \\
\hline $1 / 24 / 96$ & 18.6 & 1692 & 243592 & 0 & 19433 & 0 & 6 & 30 & 931 & 0.26 & 21180.12 & 184691 & 888750.4 \\
\hline $1 / 25 / 96$ & 12.5 & 696 & 245329 & 1737 & 19433 & 0 & 6 & 30 & 741 & & 0 & 185432 & 888750.4 \\
\hline $1 / 26 / 96$ & 13.5 & 839 & 246030 & 701 & 19433 & 0 & 6 & 30 & 844 & & 0 & 186276 & 888750.4 \\
\hline $1 / 27 / 96$ & 13.5 & 839 & 246030 & 0 & 19433 & 0 & 6 & 30 & 0 & 0.21 & 17107.02 & 186276 & 905857.4 \\
\hline $1 / 28 / 96$ & 13.5 & 839 & 246030 & 이 & 19433 & 0 & 6 & 30 & 0 & 0.02 & 1629.24 & 186276 & 907486.7 \\
\hline
\end{tabular}


Trench 31

Sheet1

\begin{tabular}{|c|c|c|c|c|c|c|c|c|c|c|c|c|c|}
\hline DATE & Pri. Level & Pri. Level & Pri/Sec & Priflow & Sec flow & Sec Flow & Sec Level & Sec Level & Total Daily & Daily Rain & Daily Rain & Tot Accu & Tot Accu \\
\hline & inches & gallons & total flow & daily & total & & inches & gallons & Water-gal & inches & gallons & Water-gal & Rain-gal \\
\hline $1 / 29 / 96$ & \begin{tabular}{|r|}
21.8 \\
\end{tabular} & 2400 & 246845 & 815 & 19433 & 0 & 5.9 & 30 & 2376 & 0.02 & 1629.24 & 188652 & 909115.9 \\
\hline $1 / 30 / 96$ & 12 & 641 & 249287 & 2442 & 19433 & 0 & 5.9 & 30 & 683 & & 0 & 189335 & 909115.9 \\
\hline $1 / 31 / 96$ & 16.9 & 1388 & 249287 & 0 & 19433 & 0 & 5.9 & 30 & 747 & & 0 & 190082 & 909115.9 \\
\hline $2 / 1 / 96$ & 19.2 & 1836 & 249287 & 0 & 19433 & 0) & 5.9 & 30 & 448 & & 0 & 190530 & 909115.9 \\
\hline $2 / 2 / 96$ & 21.4 & 2307 & 249287 & 0 & 19433 & 0 & 5.9 & 30 & 471 & & 0 & 191001 & 909115.9 \\
\hline $2 / 3 / 96$ & 21.4 & 2307 & 249287 & 0 & 19433 & 0 & 5.9 & 30 & 0 & 0.09 & 7331.58 & 191001 & 916447.5 \\
\hline $2 / 4 / 96$ & 21.4 & 2307 & 249287 & 0 & 19433 & 0 & 5.9 & 30 & 0 & 0.06 & $\begin{array}{l}8887.72 \\
\end{array}$ & 191001 & 921335.2 \\
\hline $2 / 5 / 96$ & 9.8 & 406 & 252349 & 3062 & 19433 & 이 & 5.9 & 30 & 1161 & 0.1 & 8146.2 & 192162 & 929481.4 \\
\hline $2 / 6 / 96$ & 6.5 & 75 & 252970 & 621 & 19433 & 0 & 5.9 & 30 & 290 & 0.09 & 7331.58 & 192452 & 936813 \\
\hline $2 / 7 / 96$ & 8.1 & 99 & 258600 & 5630 & 19433 & 0 & 5.9 & 30 & 5654 & 0.29 & 23623.98 & 198106 & 960437 \\
\hline 2/8/96 & 6.8 & 120 & 271562 & 14912 & 19433 & o & 5.9 & 30 & 14933 & 0.01 & 814.62 & 213039 & 961251.6 \\
\hline $2 / 9 / 96$ & 6.6 & 100 & 278698 & 11076 & 19433 & 0 & 5.9 & 30 & 11056 & & 0 & 224095 & 961251.6 \\
\hline $2 / 10 / 96$ & 6.6 & 2490 & 285196 & 6498 & 19433 & 0 & 5.9 & 30 & 8888 & & 0 & 232983 & 961251.6 \\
\hline $2 / 11 / 96$ & 6.6 & 2490 & 285196 & 0 & 19433 & 0 & 5.9 & 30 & 0 & & 0 & 232983 & 961251.6 \\
\hline $2 / 12 / 96$ & 22.2 & 2490 & 285196 & 0 & 19433 & 0 & 5.9 & 30 & 0 & & 0 & 232983 & 961251.6 \\
\hline $2 / 13 / 96$ & 22.9 & 2670 & 288371 & 3175 & 19433 & 0 & 5.9 & 30 & 3355 & & 0 & 236338 & 961251.6 \\
\hline $2 / 14 / 96$ & 24.5 & 3116 & 291815 & 3444 & 19433 & 0 & 5.9 & 30 & 3890 & & 0 & 240228 & 961251.6 \\
\hline $2 / 15 / 96$ & 26 & 3566 & 299057 & 7242 & 19433 & 0 & 5.9 & 30 & 7692 & & 0 & 247920 & 961251.6 \\
\hline $2 / 16 / 96$ & 26.3 & 3650 & 299504 & 447 & 19433 & 0 & 5.9 & 30 & 531 & & of & 248451 & 961251.6 \\
\hline 2/17/96 & 27.2 & 3650 & 299504 & 0 & 19433 & 0 & 5.9 & 30 & 0 & 0.11 & 8960.82 & 248451 & 970212.4 \\
\hline $2010 i 50$ & $2 \bar{i} .2$ & 3050 & 299504 & $\tilde{u}$ & 19405 & $\mathbf{u}$ & $0 . y$ & 30 & 01 & U.03 & 2443.86 & 2484511 & 972656.3 \\
\hline $2 / 19 / 96$ & 27.2 & 3650 & 299504 & 3200 & 19433 & 0 & 5.9 & 30 & 3200 & & 0 & 251651 & 972656.3 \\
\hline $2 / 20 / 96$ & 27.2 & 3986 & 317138 & 17634 & 19433. & 0 & 5.9 & 30 & 17970 & 0.05 & 4073.1 & 269621 & 976729.4 \\
\hline 2/21/96 & 25.4 & 3375 & 319190 & 2052 & 19433 & 0 & 5.9 & 30 & 1441 & 0.03 & 2443.86 & 271062 & 979173.2 \\
\hline 2/22/96 & 25.1 & 3290 & 323517 & 4327 & 19433 & 0 & 5.9 & 30 & 4242 & & 0 & 275304 & 979173.2 \\
\hline $2 / 23 / 96$ & 23.4 & 2800 & 327981 & 4464 & 19433 & 0 & 5.9 & 30 & 3974 & 0.01 & 814.62 & 279278 & 979987.9 \\
\hline 2/24/96 & 23.4 & 2800 & 327981 & 0 & 19433 & 0 & 5.9 & 30 & 0 & 0.13 & 10590.06 & 279278 & 990577.9 \\
\hline $2 / 25 / 96$ & 23.4 & 2800 & 327981 & 4600 & 19433 & 0 & $5 . \overline{9}$ & 30 & 4600 & 0.22 & 17921.64 & 283878 & 1008500 \\
\hline $2 / 26 / 96$ & 34.3 & 7332 & 335368 & 7387 & 19433 & 0 & 5.9 & 30 & 11919 & & 0 & 295797 & 1008500 \\
\hline $2 / 27 / 96$ & 26.2 & 3625 & 338899 & 3531 & 19433 & 0 & 5.6 & 28 & -178 & & 0 & 295619 & 1008500 \\
\hline $2 / 28 / 96$ & 26 & 3566 & 343775 & 4874 & 19435 & 2 & 5.6 & 28 & 4817 & & 0 & 300436 & 1008500 \\
\hline $2 / 29 / 96$ & 22.6 & 2570 & 346460 & 2685 & 19435 & 0 & 5.6 & 28 & 1689 & & 0 & 302125 & 1008500 \\
\hline $3 / 1 / 96$ & 24.9 & 3235 & 348520 & 2060 & 19435 & 0 & 5.6 & 28 & 2725 & & 0 & 304850 & 1008500 \\
\hline $3 / 2 / 96$ & 24.9 & 3235 & 348520 & 0 & 19435 & 0 & 5.6 & 28 & 0 & & 0 & 304850 & 1008500 \\
\hline $3 / 3 / 96$ & 24.9 & 3235 & 348520 & 0 & 19435 & 0 & 5.6 & 28 & 0 & 0.01 & 814.62 & 304850 & 1009314 \\
\hline $3 / 4 / 96$ & 22.1 & 2470 & 355335 & 6815 & 19435 & 0 & 5.5 & 28 & 6050 & 0.3 & 24438.6 & 310900 & 1033753 \\
\hline $3 / 5 / 96$ & 26.5 & 3740 & 358774 & 4689 & 19435 & 0 & 5.6 & 28 & 5959 & & 0 & 316859 & 1033753 \\
\hline $3 / 6 / 96$ & 25.2 & 3300 & 363820 & 5046 & 19435 & 0 & 5.5 & 28 & 4606 & & 0 & 321465 & 1033753 \\
\hline $3 / 7 / 96$ & 22.1 & 2450 & 367006 & 3186 & 19435 & 01 & 5.5 & 28 & 2336 & 0.1 & 8146.2 & 323801 & 1041899 \\
\hline
\end{tabular}




\begin{tabular}{|c|c|c|c|c|c|c|c|c|c|c|c|c|c|}
\hline DATE & Pri. Level & Pri. Level & Pri/Sec & Pri Flow & Sec Flow & Sec Flow & Sec Level & Sec Level & Total Daily & yaily Rain & Daily Rain & Tot Accu & Tot Accu \\
\hline & inches & gallons & total flow & daily & total & daily & inches & gallons & Water-gal & inches & gallons & Water-gal & Rain-gal \\
\hline $3 / 8 / 96$ & 20.3 & 2020 & 370420 & $\overline{3414}$ & 19435 & 0 & 5.5 & 28 & 2984 & & 0 & 326785 & 1041899 \\
\hline $3 / 9 / 96$ & 20.3 & 2020 & 370420 & 0 & 19435 & 0 & 5.5 & 28 & 0 & & 0 & 326785 & 1041899 \\
\hline $3 / 10 / 96$ & 31.9 & 6050 & 370420 & 0 & 19435 & 0 & 5.5 & 28 & 4030 & & 0 & 330815 & 1041899 \\
\hline $3 / 11 / 96$ & 21.1 & 2230 & 373024 & 2604 & 19435 & 0 & 5.5 & 28 & -1216 & 0.03 & 2443.86 & 329599 & 1044343 \\
\hline $3 / 12 / 96$ & 20.8 & 2200 & $375 \overline{740}$ & 2716 & 19435 & 0 & 5.5 & 28 & 2686 & & 0 & 332285 & 1044343 \\
\hline $3 / 13 / 96$ & 20.9 & 2220 & 378017 & 2277 & 19435 & 0 & 5.5 & 28 & 2297 & & 0 & 334582 & 1044343 \\
\hline $3 / 14 / 96$ & 19.6 & 1900 & 380474 & 2457 & 19435 & 0 & 5.5 & 28 & 2137 & & 0 & 336719 & 1044343 \\
\hline $3 / 15 / 96$ & 21.8 & 2200 & 381715 & 1241 & 19435 & 0 & 5.5 & 28 & 1541 & & 0 & 338260 & $10 \overline{44343}$ \\
\hline $3 / 16 / 96$ & 21.8 & 2200 & 381715 & 0 & 19435 & 0 & 5.5 & 28 & 0 & & 0 & 338260 & 1044343 \\
\hline $3 / 17 / 96$ & 21.8 & 2200 & 381715 & 0 & 19435 & 0 & 5.5 & 28 & 0 & & 0 & 338260 & 1044343 \\
\hline $3 / 18 / 96$ & 29.3 & 4750 & 384610 & 2895 & 19435 & 0 & 5.4 & 27 & 5444 & & 0 & 343704 & 1044343 \\
\hline $3 / 19 / 96$ & 24.5 & 3125 & 387840 & 3230 & 19435 & 0 & 5.5 & 28 & 1606 & & 0 & 345310 & 1044343 \\
\hline $3 / 20 / 96$ & 16.3 & 1250 & 391180 & 3340 & 19435 & 0 & 5.5 & 28 & 1465 & & 0 & 346775 & 1044343 \\
\hline $3 / 21 / 96$ & 16.7 & 1325 & 392200 & 1020 & 19435 & 0 & 5.4 & 27 & 1094 & & 0 & 347869 & 1044343 \\
\hline $3 / 22 / 96$ & 16.6 & 1315 & 393385 & 1185 & 19435 & 0 & 5.4 & 27 & 1175 & 0.13 & 10590,06 & 349044 & 1054933 \\
\hline $3 / 23 / 96$ & 16.6 & 1315 & 393385 & 0 & 19435 & 0 & 5.4 & 27 & 0 & & 0 & 349044 & 1054933 \\
\hline $3 / 24 / 96$ & 16.6 & 1315 & 393385 & 0 & 19435 & $\overline{0}$ & 5.4 & 27 & 0 & & 0 & 349044 & 1054933 \\
\hline $3 / 25 / 96$ & 26.7 & 3750 & 395630 & 2245 & 19435 & 0 & 5.4 & 27 & 4680 & & 0 & 353724 & 1054933 \\
\hline $3 / 26 / 96$ & 14.5 & 1000 & 398769 & 3139 & 19435 & 0 & 5.5 & 28 & 390 & & 0 & 354114 & $10 \overline{54933}$ \\
\hline $3 / 27 / 96$ & 15.5 & 1130 & 400096 & 1327 & 19435 & 0 & 5.5 & 28 & 1457 & & 0 & 355571 & 1054933 \\
\hline $3 / 28 / 96$ & 13.1 & 870 & 401540 & 1444 & 10435 & $\bar{v}$ & 5.5 & $\tilde{z} \hat{U}$ & $\therefore: 104$ & & ה & 350750 & 1054023 \\
\hline $3 / 29 / 96$ & 14.4 & 980 & 402160 & 620 & 19435 & 0 & 5.5 & 28 & 730 & & 0 & 357485 & 1054933 \\
\hline $3 / 30 / 96$ & 14.4 & 980 & 402160 & 0 & 19435 & 0 & 5.5 & 28 & 0 & & 0 & 357485 & 1054933 \\
\hline $3 / 31 / 96$ & 14.4 & 980 & 402160 & 0 & 19435 & 0 & 5.5 & 28 & 0 & 0.26 & 21180.12 & 357485 & 1076113 \\
\hline $4 / 1 / 96$ & 24.3 & 3030 & 403360 & 1200 & 19435 & 0 & 5.5 & 28 & 3250 & 0.12 & 9775.44 & 360735 & 1085888 \\
\hline $4 / 2 / 96$ & 19.9 & 1990 & 406750 & 3390 & 19435 & 0 & 5.5 & 28 & 2350 & & 0 & 363085 & 1085888 \\
\hline $4 / 3 / 96$ & 17.2 & 1440 & 409400 & 2650 & 19435 & 0 & 5.3 & 27 & 2099 & & 0 & 365184 & 1085888 \\
\hline $4 / 4 / 96$ & 16.4 & 1275 & 410460 & 1060 & 19435 & 0 & 5.5 & 28 & 896 & & 0 & 366080 & 1085888 \\
\hline $4 / 5 / 96$ & 15.6 & $11 \overline{60}$ & 411780 & 1320 & 19435 & 0 & 5.5 & 28 & 1205 & & 0 & 367285 & 1085888 \\
\hline $4 / 6 / 96$ & 15.6 & 1160 & 411780 & 0 & 19435 & 0 & 5.5 & 28 & 0 & & 0 & 367285 & 1085888 \\
\hline $4 / 7 / 96$ & 15.6 & 1160 & 411780 & 0 & 19435 & 0 & 5.5 & 28 & 0 & & 0 & 367285 & 1085888 \\
\hline $4 / 8 / 96$ & 24.8 & 3200 & 413094 & 1314 & 19435 & 0 & 5.5 & 28 & 3354 & & 0 & 370639 & 1085888 \\
\hline $4 / 9 / 96$ & 17 & 1406 & 416010 & 2916 & 19435 & 0 & 5.5 & 28 & 1122 & & 0 & 371761 & 1085888 \\
\hline $4 / 10 / 96$ & 14.4 & 980 & 418140 & 2130 & 19435 & 0 & 5.5 & 28 & $170 \overline{4}$ & & 0 & 373465 & 1085888 \\
\hline $4 / 11 / 96$ & 14 & 919 & 418880 & 740 & 19435 & 0 & 5.5 & 28 & 679 & & 0 & 374144 & 1085888 \\
\hline $4 / 12 / 96$ & 14 & 919 & 419540 & 660 & 19435 & 0 & 5.5 & 28 & 660 & & 0 & 374804 & 1085888 \\
\hline $4 / 13 / 96$ & 14 & 919 & 419540 & 0 & 19435 & 0 & 5.5 & 28 & 0 & & 0 & 374804 & 1085888 \\
\hline $4 / 14 / 96$ & 14 & 919 & 419540 & 0 & 19435 & 0 & 5.5 & 28 & 0 & & 0 & 374804 & 1085888 \\
\hline $4 / 15 / 96$ & 18.96 & 1790 & 420570 & 1030 & 19435 & 0 & 5.5 & 28 & 1901 & & 0 & 376705 & 1085888 \\
\hline
\end{tabular}


Trench 31

Sheet1

\begin{tabular}{|c|c|c|c|c|c|c|c|c|c|c|c|c|c|}
\hline DATE & Pri. Level & Pri. Level & Pri/Sec & Pri Flow & Sec Flow & Sec Flow & Sec Level & Sec Level & Total Daily & Daily Rain & \begin{tabular}{|l|} 
Daily Rain \\
\end{tabular} & Tot Accu & Tot Accu \\
\hline & inches & gallons & total flow & daily & total & daily & inches & gallons & Water-gal & inches & gallons & Water-gal & Rain-gal \\
\hline $4 / 16 / 96$ & 13 & 774 & \begin{tabular}{|l|}
422550 \\
\end{tabular} & 1980 & 19435 & 0 & 5.5 & 28 & 964 & 0.01 & 814.62 & 377669 & 1086703 \\
\hline $4 / 17 / 96$ & 13.7 & 870 & 423160 & 610 & 19435 & 0 & 5.6 & 28 & 706 & & 0 & 378375 & 1086703 \\
\hline $4 / 18 / 96$ & 11.7 & 610 & 423770 & 610 & 19435 & 0 & 5.6 & 28 & 350 & & 0 & 378725 & 1086703 \\
\hline $4 / 19 / 96$ & 10.8 & 510 & 424720 & 950 & 19435 & 0 & 5.6 & 28 & 850 & & 0 & 379575 & 1086703 \\
\hline $4 / 20 / 96$ & 10.8 & 510 & 424720 & 0 & 19435 & 0 & 5.5 & 28 & 0 & & 0 & 379575 & 1086703 \\
\hline $4 / 21 / 96$ & 10.8 & 510 & 424720 & 0 & 19435 & 0 & 5.6 & 28 & 0 & & 0 & 379575 & 1086703 \\
\hline $4 / 22 / 96$ & 18.5 & 1695 & 426650 & 1930 & 19435 & 0 & 5.6 & 28 & 3115 & 0.28 & 22809.36 & 382690 & 1109512 \\
\hline $4 / 23 / 96$ & 12.4 & 685 & 426670 & 20 & 19435 & 0 & 5.6 & 28 & -990 & 0.02 & 1629.24 & 381700 & 1111142 \\
\hline $4 / 24 / 96$ & 13.4 & 820 & 427360 & 690 & 19435 & 0 & 5.6 & 28 & 825 & & 0 & 382525 & 1111142 \\
\hline $4 / 25 / 96$ & 12.7 & 715 & 428170 & 810 & 19435 & 0 & 5.6 & 28 & 705 & & 0 & 383230 & 1111142 \\
\hline $4 / 26 / 96$ & 12.2 & 660 & 428800 & 630 & 19435 & 0 & 5.6 & 28 & 575 & & 0 & 383805 & 1111142 \\
\hline $4 / 27 / 96$ & 12.2 & 660 & 428800 & 0 & 19435 & 0 & $5 . \overline{6}$ & 28 & 0 & & 0 & 383805 & 1111142 \\
\hline $4 / 28 / 96$ & 12.2 & 660 & 428800 & 0 & 19435 & 0 & 5.6 & 28 & 0 & & 0 & 383805 & 1111142 \\
\hline $4 / 29 / 96$ & 17.4 & 1480 & 429570 & 770 & 19435 & 0 & 5.7 & 29 & 1591 & & 0 & 385396 & 1111142 \\
\hline $4 / 30 / 96$ & 11.1 & 540 & 430950 & 1380 & 19435 & 0 & 5.7 & 29 & 440 & & 0 & 385836 & 1111142 \\
\hline $5 / 1 / 96$ & 9.1 & 334 & 431630 & 680 & 19435 & 0 & 5.7 & 29 & 474 & & 0 & 386310 & 1111142 \\
\hline $5 / 2 / 96$ & 9.2 & 344 & 432380 & 750 & 19435 & 0 & 5.7 & 29 & 760 & & 0 & 387070 & 1111142 \\
\hline $5 / 3 / 96$ & 10.2 & 446 & 432495 & 115 & 19435 & 0 & 5.7 & 29 & 217 & & 0 & 387287 & 1111142 \\
\hline $5 / 4 / 96$ & 10.2 & 446 & 432495 & 0 & 19435 & 0 & 5.7 & 29 & 0 & & 0 & 387287 & 1111142 \\
\hline $5 / 5 / 96$ & 10.2 & 446 & 432495 & 0 & 19435 & 0 & 5.7 & 29 & 0 & & 01 & 387287 & 1111142 \\
\hline $5 / 6 / 90$ & 10.4 & 1700 & $43<Y 7 s$ & 418 & 19435 & u & 0.11 & $2 \mathrm{y}$ & $10 / 2$ & & 0 & 388359 & 1111142 \\
\hline $5 / 7 / 96$ & 17.1 & 1420 & 432913 & 0 & 19435 & 0 & 5.7 & 29 & 320 & & 0 & 388679 & 1111142 \\
\hline $5 / 8 / 96$ & 19 & 1796 & 432950 & 37 & 19435 & 0 & 5.8 & 29 & 413 & & 0 & 389092 & 1111142 \\
\hline $5 / 9 / 96$ & 9.4 & 365 & 434650 & 1700 & 19435 & 0 & 5.8 & 29 & 269 & & 0 & 389361 & 1111142 \\
\hline $5 / 10 / 96$ & 8.3 & 254 & 435068 & 418 & 19435 & 0 & 5.8 & 29 & 307 & & 0 & 389668 & 1111142 \\
\hline $5 / 11 / 96$ & 8.3 & 254 & 435068 & 0 & 19435 & 0 & 5.8 & 29 & 0 & & 0 & 389668 & 1111142 \\
\hline $5 / 12 / 96$ & 8.3 & 254 & 435068 & 0 & 19435 & 0 & 5.8 & 29 & 0 & 0.14 & 11404.68 & 389668 & 1122546 \\
\hline $5 / 13 / 96$ & 15.8 & 1195 & 435100 & 32 & 19435 & 0 & 5.8 & 29 & 973 & & 0 & 390641 & 1122546 \\
\hline $5 / 14 / 96$ & 10.1 & 437 & 436182 & 1082 & 19435 & 0 & 5.9 & 30 & 325 & & 0 & 390966 & 1122546 \\
\hline $5 / 15 / 96$ & 10.1 & 437 & 436182 & 0 & 19435 & 0 & 5.9 & 30 & 0 & 0.06 & 4887.72 & 390966 & 1127434 \\
\hline $5 / 16 / 96$ & 11.8 & 619 & 436625 & 443 & 19435 & 0 & 5.9 & 30 & 625 & & 0 & 391591 & 1127434 \\
\hline $5 / 17 / 96$ & 11.8 & 619 & 436625 & 0 & 19435 & 0 & 5.9 & 30 & 0 & 0.12 & 9775.44 & 391591 & 1137210 \\
\hline $5 / 18 / 96$ & 11.8 & 619 & 436625 & 0 & 19435 & 0 & 5.9 & 30 & 0 & & 0 & 391591 & 1137210 \\
\hline 5/19/96 & 11.8 & 619 & 436625 & 0 & 19435 & 0 & 5.9 & 30 & 0 & & 0 & 391591 & 1137210 \\
\hline $5 / 20 / 96$ & 6.3 & 76 & 437273 & 648 & 19435 & 0 & 6.1 & 34 & 109 & & 0 & 391700 & 1137210 \\
\hline $5 / 21 / 96$ & 9.7 & 395 & 438499 & 1226 & 19435 & 0 & 6.2 & 38 & 1549 & 0.2 & 16292.4 & 393249 & 1153502 \\
\hline $5 / 22 / 96$ & 12.4 & 694 & 438499 & 0 & 19435 & 0 & 6.3 & 41 & 302 & & 0 & 393551 & 1153502 \\
\hline $5 / 23 / 96$ & 14.5 & 995 & 439500 & 1001 & 19435 & 0 & 6.3 & 41 & 1302 & & \begin{tabular}{l|l}
0 \\
\end{tabular} & 394853 & 1153502 \\
\hline $5 / 24 / 96$ & 14.5 & 995 & 439500 & 0 & 19435 & 0 & 6.3 & 41 & 0 & & 0 & 394853 & 1153502 \\
\hline
\end{tabular}


Trench 31

Sheet1

\begin{tabular}{|c|c|c|c|c|c|c|c|c|c|c|c|c|c|}
\hline DATE & Pri. Level & Pri. Level & $\mathrm{Pri} / \mathrm{Sec}$ & Pri Flow & Sec Flow & Sec Flow & Sec Level & \begin{tabular}{|l|} 
Sec Level \\
allons
\end{tabular} & Total Daily & Daily Rain & Daily Rain & Tot Accu & Tot Accu \\
\hline & & & & & & & & & & & & Water-gal & \\
\hline $5 / 25 / 96$ & 14.5 & 995 & 439500 & 0] & 19435 & 0 & 6.3 & 41 & D & & 0 & 394853 & 1153502 \\
\hline $5 / 26 / 96$ & 14.5 & 995 & 439500 & 0 & 19435 & 0 & 6.3 & 41 & 0 & & 0 & 394853 & 1153502 \\
\hline $5 / 27 / 96$ & 14.5 & 995 & 439500 & 0 & 19435 & 0 & 6.3 & 41 & ㅇ. & & 0 & 394853 & 1153502 \\
\hline $5 / 28 / 96$ & 16.2 & 1262 & 439500 & 0 & 19435 & 0 & 6.7 & 57 & 283 & 0.01 & 814.62 & 395136 & 1154317 \\
\hline $5 / 29 / 96$ & 17.7 & 1538 & 439500 & 0 & 19435 & 0 & 6.8 & 60 & 279 & 0.09 & 7331.58 & 395415 & 1161648 \\
\hline $5 / 30 / 96$ & 17.7 & 1538 & 439500 & o & 19435 & 0 & 6.8 & 60 & 0 & & 0 & 395415 & 1161648 \\
\hline $5 / 31 / 96$ & 20.7 & 2379 & 439500 & 0 & 19435 & 0 & 7 & 68. & 849 & & 0 & 396264 & 1161648 \\
\hline $6 / 1 / 96$ & 20.7 & 2379 & 439500 & 0 & 19435 & 0 & 7 & 68 & 의 & & 0 & 396264 & 1161648 \\
\hline $6 / 2 / 96$ & 20.7 & 2379 & 439500 & 0 & 19435 & 0 & 7 & 68 & 0 & & 0 & 396264 & 1161648 \\
\hline $6 / 3 / 96$ & 23.9 & 2948 & 439500 & 0 & 19435 & 0 & 7.4 & 84 & 585 & & 0 & 396849 & 1161648 \\
\hline $6 / 4 / 96$ & 24.5 & 3119 & 439500 & 0 & 19435 & 0 & 7.4 & 84 & 171 & & 0 & 397020 & 1161648 \\
\hline $6 / 5 / 96$ & 24.5 & 3119 & 439500 & 0 & 19435 & 0 & 7.4 & 84 & 0 & & 0 & 397020 & 1161648 \\
\hline $6 / 6 / 96$ & 10.3 & 457 & 442528 & 3028 & 19435 & 0 & 7.7 & 97 & 379 & & 0 & 397399 & 1161648 \\
\hline $6 / 7 / 96$ & 10.3 & 457 & 442528 & 0 & 19435 & 0 & 7.7 & $97]$ & 0 & & 0 & 397399 & 1161648 \\
\hline $6 / 8 / 96$ & 10.3 & 457 & 442528 & 0 & 19435 & 0 & 7.7 & 97 & 0 & & 0 & 397399 & 1161648 \\
\hline $6 / 9 / 96$ & 10.3 & 457 & 442528 & 0 & 19435 & 0 & 7.7 & 97 & 0 & & 0 & 397399 & 1161648 \\
\hline $6 / 10 / 96$ & 10.3 & 457 & 442528 & 0 & 19435 & 0 & 7.7 & 97 & 0 & & 0 & 397399 & 1161648 \\
\hline $6 / 11 / 96$ & 10.3 & 457 & 442528 & 0 & 19435 & 0 & 7.7 & 97 & o & & 0 & 397399 & 1161648 \\
\hline $6 / 12 / 96$ & 20.8 & 2178 & 442528 & 0 & 19435 & 0 & 9 & 154 & 1778 & & 0 & 399177 & 1161648 \\
\hline $6 / 13 / 96$ & 22.5 & 2571 & 442528 & 0 & 19435 & 0 & 9.3 & 170 & 409 & & 0 & 399586 & 1161648 \\
\hline $6 / 14 / 96$ & 23 & $\angle O Y O$ & $44 \angle 5 \angle O$ & $\hat{v}$ & $\{3+35$ & 3 & 2.5 & 180 & 125 & & n & 200731 & 11F1GAR \\
\hline $6 / 15 / 96$ & 23 & 2696 & 442528 & 0 & 19435 & 0 & 9.5 & 180 & 0 & & 0 & 399721 & 1161648 \\
\hline $6 / 16 / 96$ & 23 & 2696 & 442528 & 0 & 19435 & 0 & 9.5 & 180 & 0 & & 0 & 399721 & 1161648 \\
\hline $6 / 17 / 96$ & 23 & 2696 & 442528 & 0 & 19435 & 0 & 9.5 & 180 & 0 & & 0 & 399721 & 1161648 \\
\hline $6 / 18 / 96$ & 26.9 & 3881 & 442528 & 0 & 19435 & 0 & 10.8 & 252 & 1257 & & 0 & 400978 & 1161648 \\
\hline $6 / 19 / 96$ & & & & & & & & & & & & & \\
\hline $6 / 20 / 96$ & & & & & & & & & & & & & \\
\hline
\end{tabular}


WHC-SD-WM-TI-767, Rev. 0

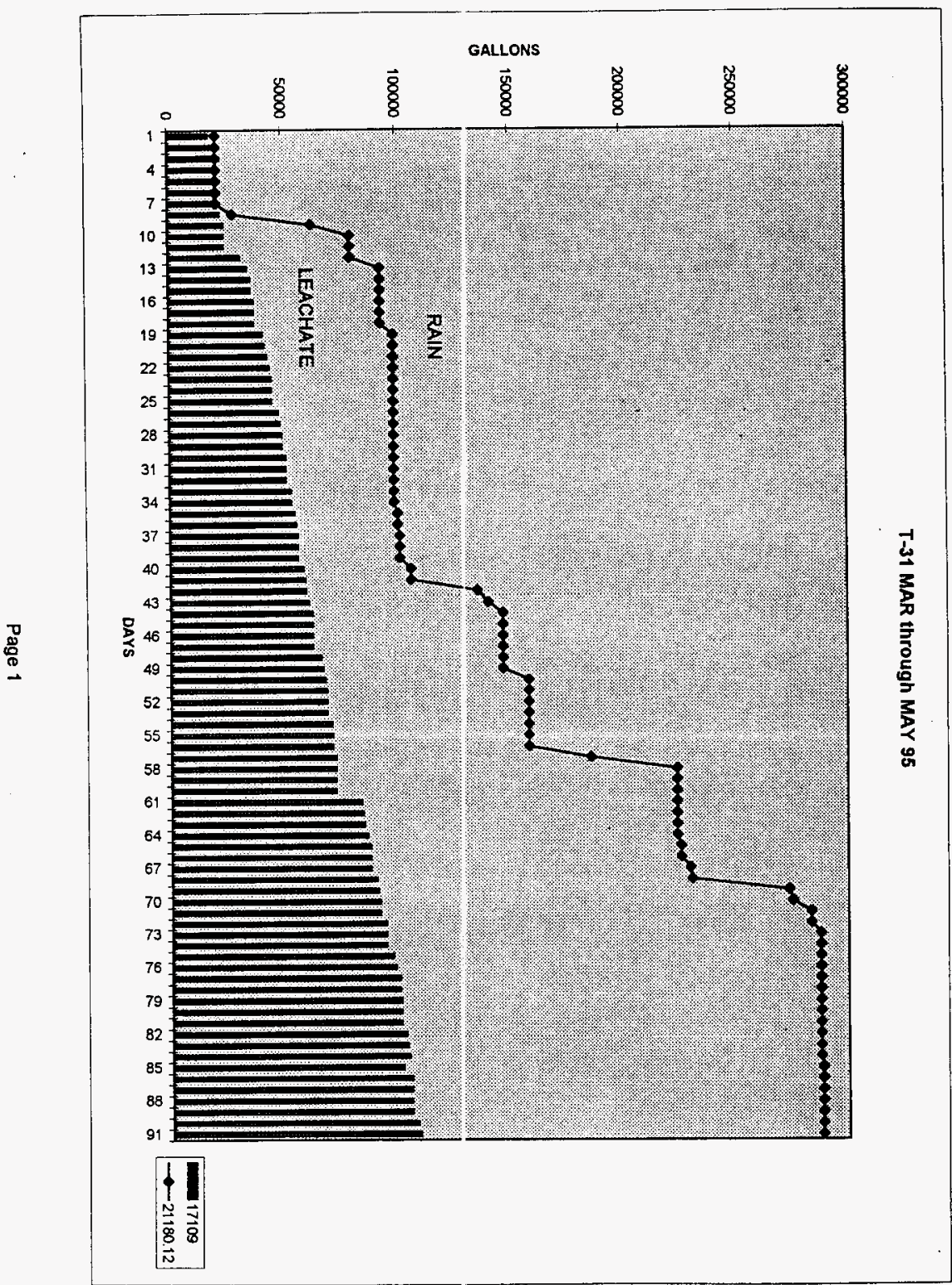


WHC-؟D-WM-TI-767, Rev. 0

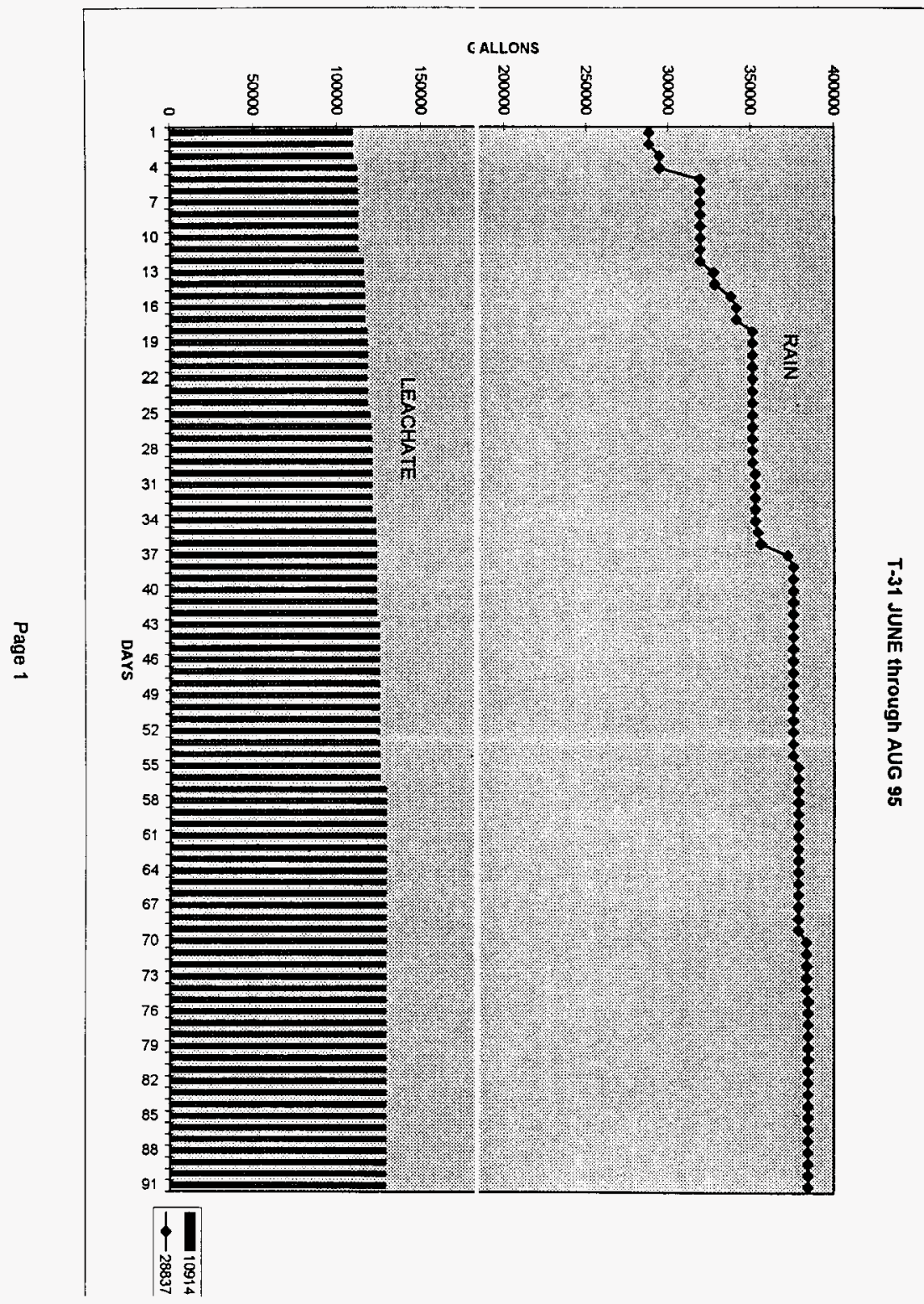


WHC-SD-WM-TI-767, Rev. 0

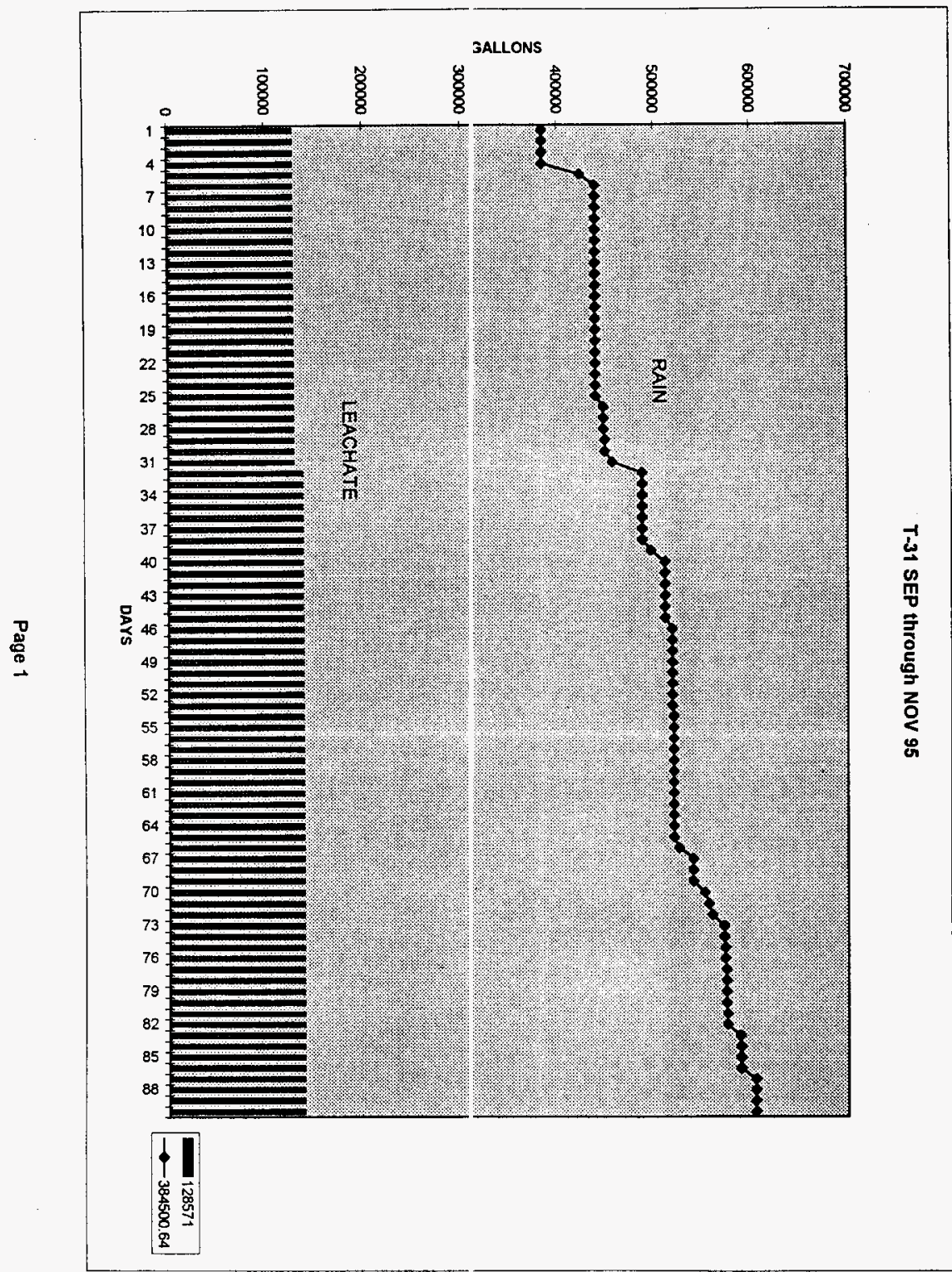


WHC-jD-WM-TI-767, Rev. 0

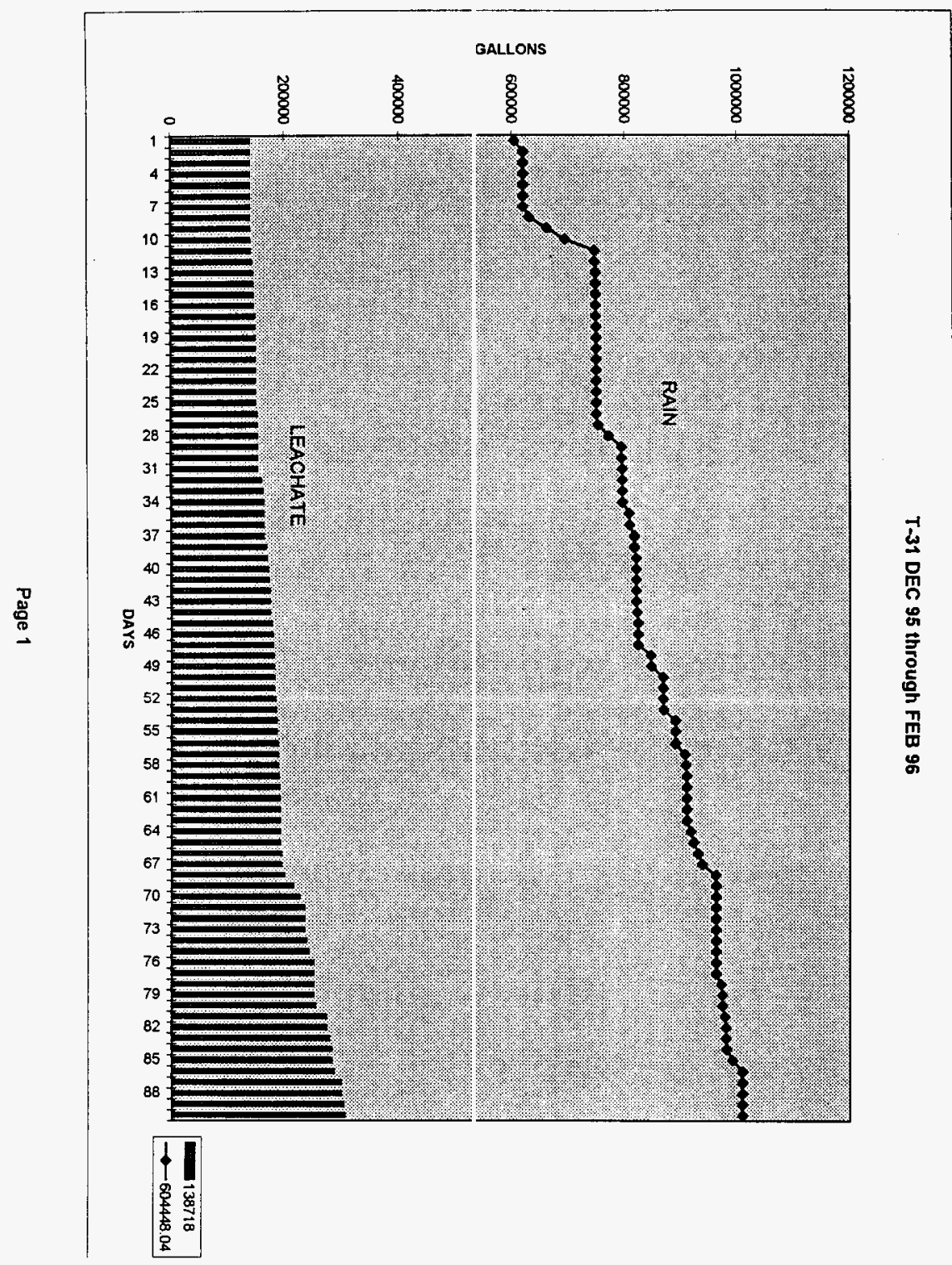


WHC-SD-WM-TI-767, Rev. 0

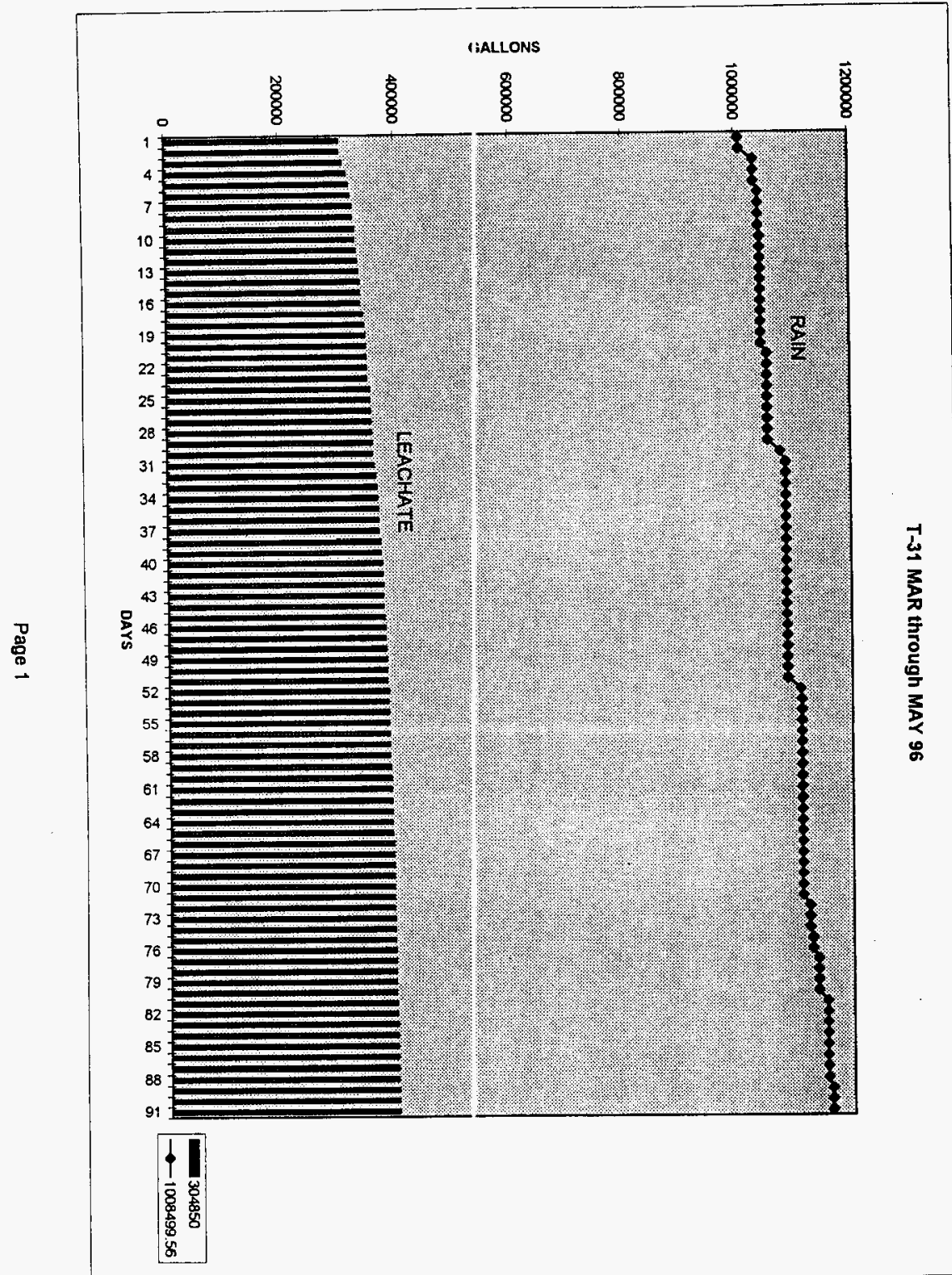


WHC-5D-WM-TI-767, Rev. O

WHC-SD-WM-TI-767, Rev. 0

Appendix C

Trench 34 Data and Graphs

C-1 
Trench 34

Sheet1

\begin{tabular}{|c|c|c|c|c|c|c|c|c|c|c|c|c|c|}
\hline \multirow[t]{3}{*}{ DATE } & Pri. Level & Pri. Level & $\mathrm{Pri} / \mathrm{Sec}$ & Pri Flow & Sec Flow & Sec Flow & Sec Level & \begin{tabular}{|l|} 
Sec Level \\
gallons
\end{tabular} & $\mid \frac{\text { Total Daily }}{\mid \text { Water-aal }}$ & Daily Rain & \begin{tabular}{|l} 
Daily Rain \\
\end{tabular} & Tot Accu & Tot Accu \\
\hline & inches & gallons & total flow & & & & & & & & & Water-gal & Rain-gal \\
\hline & 25 & 3199 & 52468 & & 2454 & & 9.4 & 206 & & 0.23 & \begin{tabular}{|l|}
18736.26 \\
\end{tabular} & & 18736.26 \\
\hline 2/13/95 & 35.6 & 7894 & 56880 & 4413 & 2453 & -1 & 9.36 & 204 & 9105 & & 0 & 9105 & 18736.26 \\
\hline $2 / 14 / 95$ & 21.9 & 2326 & 62834 & 5954 & 2453 & 0 & 9.35 & 203 & 385 & & 0 & 9490 & 18736.26 \\
\hline $2 / 15 / 95$ & 20.51 & 2025 & 65337 & 2503 & 2453 & 0 & 9.34 & 203 & 2202 & & 0 & 11692 & 18736.26 \\
\hline $2 / 16 / 95$ & 26.8 & 3748 & 65337 & 0 & 2453 & 0 & 9.3 & 201 & 1721 & & 0 & 13413 & 18736.26 \\
\hline $2 / 17 / 95$ & 31.68 & 5816 & 65338 & 1 & 2453 & 0 & 9.33 & 203 & 2071 & & 0 & 15484 & 18736.26 \\
\hline $2 / 18 / 95$ & 31.68 & 5816 . & 65338 & 0 & 2453 & 0 & 9.33 & 203 & o) & 0.03 & 2443.86 & 15484 & 21180.12 \\
\hline 2/19/95 & 31.68 & 5816 & 65338 & 0 & 2453 & 0 & 9.33 & 203 & 0 & & 0 & 15484 & 21180.12 \\
\hline $2 / 20 / 95$ & 31.68 & 5816 & 65338 & 0 & 2453 & 0 & 9.33 & 203 & o. & & 0 & 15484 & 21180.12 \\
\hline 2/21/95 & 35.1 & 7622 & 67310 & 1972 & 2453 & 0 & 9.73 & 203 & 3778 . & & 0 & 19262 & 21180.12 \\
\hline 2/22/95 & 37.4 & 9059 & 70153 & 2843 & 2453 & 이 & 9.8 & 225 & 4302 & & 0 & 23564 & 21180.12 \\
\hline $2 / 23 / 95$ & 38.7 & 9924 & 71220 & 1067 & 2453 & 0 & 9.95 & 232 & 1939 & & 0 & 25503 & 21180.12 \\
\hline $2 / 24 / 95$ & 7.5 & 356 & 80472 & 9084 & 2621 & 168 & 6.07 & 61 & -487 & & 0 & 25016 & 21180.12 \\
\hline $2 / 25 / 95$ & 7.5 & 356 & 80472 & 0 & 2621 & 0 & 6.07 & 61 & 0 & & 0 & 25016 & 21180.12 \\
\hline $2 / 26 / 95$ & 7.5 & 356 & 80472 & 0 & 2621 & 0 & 6.07 & 61 & 0 & & 0 & 25016 & 21180.12 \\
\hline $2 / 27 / 95$ & 27.75 & 4064 & 81776 & 1303 & 2622 & 1 & 6.11 & 61 & 5012 & & 0 & 30028 & 21180.12 \\
\hline $2 / 28 / 95$ & 30.2 & 5135 & 81776 & 1 & 2621 & -1 & 6 & 59 & 1069 & & 0 & 31097 & 21180.12 \\
\hline $3 / 1 / 95$ & 32.2 & 6063 & 81777 & 2 & 2620 & -1 & 6 & 59 & 929 & & ㅇ. & 32026 & 21180.12 \\
\hline $3 / 2 / 95$ & 22.6 & 2486 & 86345 & 4567 & 2621 & 1 & 6.04 & 60 & 992 & & 0 & 33018 & 21180.12 \\
\hline $3 / 3 / 95$ & 17.3 & 1457 & 88535 & 2190 & 2621 & 0 & 6 & 59 & 1160 & & 0 & 34178 & 21180.12 \\
\hline $3 / 4 / 9 \mathrm{~b}$ & 11,3 & 1456 & 80530 & u & $20<1$ & $\because$ & 미 & jy & uj & & uj & 34110 & $\angle 1100.12$ \\
\hline $3 / 5 / 95$ & 17.3 & 1457 & 88535 & 0 & 2621 & 0 & 6 & 59 & 0 & & 아 & 34178 & 21180.12 \\
\hline $3 / 6 / 95$ & 26.9 & 3779 & 88535 & 0 & 2621 & 0 & 5.94 & 49 & 2312 & & 0 & 36490 & 21180.12 \\
\hline $3 / 7 / 95$ & 26.9 & 3779 & 88535 & 0 & 2621 & 0 & 5.94 & 49 & 0 & & 요 & 36490 & 21180.12 \\
\hline $3 / 8 / 95$ & 30.52 & 5275 & 88535 & 0 & 2621 & 0 & 5.93 & 48 & 1495 & & o & 37985 & 21180.12 \\
\hline $3 / 9 / 95$ & 32.03 & 5984 & 88535 & 0 & 2621 & 0 & 5.9 & 48 & 709 & 0.09 & 7331.58 & 38694 & 28511.7 \\
\hline $3 / 10 / 95$ & 21.77 & 2269 & 94257 & 5722 & 2621 & 0 & 5.88 & 47 & 2006 & 0.42 & 34214.04 & 40700 & 62725.74 \\
\hline 3/11/95 & 21.77 & 2269 & 94257 & 0 & 2621 & 0 & 5.88 & 47 & 0 & 0.21 & 17107.02 & 40700 & 79832.76 \\
\hline $3 / 12 / 95$ & 21.77 & 2269 & 94257 & 0 & 2621 & 요 & 5.88 & 47 & 0 & & 으 & 40700 & 79832.76 \\
\hline $3 / 13 / 95$ & 35.37 & 7769 & 96327 & 2070 & 2621 & 0 & 5.91 & 48 & 7571 & & 0 & 48271 & 79832.76 \\
\hline $3 / 14 / 95$ & 27.64 & 4029 & 101720 & 5393 & 2621 & 0 & 5.87 & 47 & 1652 & 0.16 & 13033.92 & 49923 & 92866.68 \\
\hline $3 / 15 / 95$ & 21.16 & 2152 & 105242 & 3522 & 2621 & 0 & 5.89 & 47 & 1645 & & 0 & 51568 & 92866.68 \\
\hline $3 / 16 / 95$ & 22.64 & 2495 & 107270 & 2028 & 2621 & 0 & 5.83 & 46 & 2370 & & 0 & 53938 & 92866.68 \\
\hline $3 / 17 / 95$ & 27.21 & 3891 & 107270 & 0 & 2621 & 0 & 5.84 & 46 & 1396 & & 0 & 55334 & 92866.68 \\
\hline $3 / 18 / 95$ & 27.21 & 3891 & 107270 & 0 & 2621 & 0 & 5.79 & 45 & -1 & & 0 & 55333 & 92866.68 \\
\hline $3 / 19 / 95$ & 27.21 & 3891 & 107270 & 0 & 2621 & 0 & 5.79 & 45 & 0 & & o) & 55333 & 92866.68 \\
\hline $3 / 20 / 95$ & 28.65 & 4418 & 110795 & 3525 & 2621 & 0 & 5.79 & 45 & 4052 & 0.07 & 5702.34 & 59385 & 98569.02 \\
\hline $3 / 21 / 95$ & 16.4 & 1329 & 115222 & 4427 & 2621 & 0 & 5.8 & 45 & 1338 & & 0 & 60723 & 98569.02 \\
\hline $3 / 22 / 95$ & 22.44 & 2449 & 115222 & 0 & 2621 & 0 & 5.82 & 46 & 1121 & & 0 & 61844 & 98569.02 \\
\hline
\end{tabular}

Page 1 


\begin{tabular}{|c|c|c|c|c|c|c|c|c|c|c|c|c|c|}
\hline DATE & $\begin{array}{l}\text { Pri. Level } \\
\text { inches }\end{array}$ & \begin{tabular}{|l|} 
Pri. Level \\
gallons \\
\end{tabular} & \begin{tabular}{|l} 
Pri/Sec \\
total flow \\
\end{tabular} & $\begin{array}{l}\text { Pri Flow } \\
\text { daily }\end{array}$ & \begin{tabular}{|l|} 
Sec Flow \\
total
\end{tabular} & \begin{tabular}{|l} 
Sec Flow \\
daily \\
\end{tabular} & \begin{tabular}{|l|} 
Sec Level \\
inches \\
\end{tabular} & \begin{tabular}{|l|} 
Sec Level \\
gallons \\
\end{tabular} & \begin{tabular}{|l|} 
Total Daily \\
Water-gal \\
\end{tabular} & \begin{tabular}{|l|} 
Daily Rain \\
inches
\end{tabular} & \begin{tabular}{|l|} 
Daily Rain \\
gallons
\end{tabular} & \begin{tabular}{|l|} 
Tot Accu \\
Water-gal \\
\end{tabular} & $\begin{array}{l}\text { Tot Accu } \\
\text { Rain-gal } \\
\end{array}$ \\
\hline $3 / 23 / 95$ & $\begin{array}{r}\text { inches } \\
26.26\end{array}$ & & \begin{tabular}{|r|} 
total flow \\
115222 \\
\end{tabular} & daily & total & 0 & 5.79 & 45 & 1133 & & 0 & 62977 & \begin{tabular}{|l|}
98569.02 \\
\end{tabular} \\
\hline $\begin{array}{l}3 / 23 / 95 \\
3 / 24 / 95 \\
\end{array}$ & $\begin{array}{r}26.26 \\
15.92 \\
\end{array}$ & $\begin{array}{r}3583 \\
1270 \\
\end{array}$ & $\begin{array}{r}115222 \\
118598 \\
\end{array}$ & 3376 & 2621 & 0 & 5.81 & 45 & 1063 & & 0 & 64040 & 98569.02 \\
\hline $3 / 25 / 95$ & 15.92 & 1270 & 118598 & 0 & 2621 & 0 & 5.81 & 45 & 0 & & 0 & 64040 & 98569.02 \\
\hline 3/26/95 & 15.92 & 1270 & 118598 & 0 & 2621 & 0 & 5.81 & 45 & 0 & & 0 & 64040 & 98569.02 \\
\hline $3 / 27 / 95$ & 28.87 & 4497 & 118599 & 1 & 2621 & 0 & 5.71 & 43 & 3226 & & 0 & 67266 & 98569.02 \\
\hline $3 / 28 / 95$ & 28.87 & 4497 & 118599 & 0 & 2621 & 0 & 5.72 & 43 & 0 & & 0 & 67266 & 98569.02 \\
\hline $\begin{array}{l}3 / 29 / 95 \\
3 / 30 / 95 \\
\end{array}$ & 30.71 & 5358 & 118599 & 0 & 2621 & 0 & 5.7 & 43 & 861 & & 0 & 68127 & 98569.02 \\
\hline $\begin{array}{l}3 / 30 / 95 \\
3 / 31 / 95 \\
\end{array}$ & 30.71 & 5358 & 118599 & 0 & 2621 & 0 & 5.7 & 43 & 0 & & 0 & 68127 & 98569.02 \\
\hline $\begin{array}{r}3 / 31 / 95 \\
4 / 1 / 95 \\
\end{array}$ & 33.69 & 6839 & 118599 & 0 & 2621 & 0 & 5.69 & 42 & 1480 & & 0 & 69607 & 98569.02 \\
\hline $\begin{array}{l}4 / 1 / 95 \\
4 / 2 / 95 \\
\end{array}$ & 33.69 & 6839 & 118599 & 0 & 2621 & 0 & 5.69 & 42 & 0 & & 0 & 69607 & 98569.02 \\
\hline $\begin{array}{l}4 / 2 / 95 \\
4 / 3 / 95 \\
\end{array}$ & 33.69 & 6839 & 118599 & 0 & 2621 & 0 & 5.69 & 42 & 0 & & 0 & 69607 & 98569.02 \\
\hline $\begin{array}{l}4 / 3 / 95 \\
4 / 4 / 95 \\
\end{array}$ & 36.74 & 8618 & 118599 & 0 & 2621 & 0 & 5.74 & 44 & 1781 & & 0 & 71388 & 98569.02 \\
\hline $\begin{array}{l}4 / 4 / 95 \\
4 / 5 / 95 \\
\end{array}$ & 36.74 & 8618 & 118599 & 0 & 2621 & of & 5.74 & 44 & 0 & & 0 & 71388 & 98569.02 \\
\hline 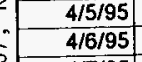 & 19.44 & 1828 & 126692 & 8093 & 2621 & 0 & 5.75 & 44. & 1303 & 0.02 & 1629.24 & 72691 & 100198.3 \\
\hline $\begin{array}{r}4 / 6 / 95 \\
4 / 7 / 95 \\
\end{array}$ & 22.7 & 2509 & 126691 & -1 & 2621 & 0 & 5.73 & 43 & 679 & & 0 & 73370 & 100198.3 \\
\hline $\begin{array}{l}4 / 7 / 95 \\
4 / 8 / 95 \\
\end{array}$ & $\begin{array}{l}12.53 \\
12.53 \\
\end{array}$ & 830 & 128888 & 2197 & 2621 & 0 & 5.72 & 43 & 518 & 0.01 & 814.62 & 73888 & 101012.9 \\
\hline $\begin{array}{r}4 / 8 / 95 \\
4 / 9 / 95 \\
\end{array}$ & \begin{tabular}{|l|}
12.53 \\
12.53
\end{tabular} & 830 & 128888 & $\begin{array}{l}0 \\
0\end{array}$ & $\begin{array}{l}2621 \\
2621\end{array}$ & 0 & 5.72 & 43 & 0 & & 0 & 73888 & 101012.9 \\
\hline $4 / 10 / 95$ & $\begin{array}{l}12.53 \\
19.16 \\
\end{array}$ & 830 & $\frac{128888}{129677}$ & $\begin{array}{r}0 \\
7899 \\
\end{array}$ & $\begin{array}{l}2621 \\
2621\end{array}$ & 0 & 5.7 & 43 & 0 & & 0 & 73888 & 1010129 \\
\hline $4 / 41 / 95$ & $\begin{array}{l}19.16 \\
21.63 \\
\end{array}$ & 1780 & $\begin{array}{l}129677 \\
129678\end{array}$ & $\begin{array}{r}789 \\
-1\end{array}$ & $\begin{array}{l}2621 \\
2621 \\
\end{array}$ & 0 & 5.7 & 43 & 1739 & 0.06 & 4887.72 & 75627 & 105900.6 \\
\hline $4 / 12 / 95$ & $\begin{array}{r}21.63 \\
11.3 \\
\end{array}$ & 2242 & $\begin{array}{l}129678 \\
131792\end{array}$ & $\begin{array}{r}1] \\
2114\end{array}$ & $\begin{array}{l}2621 \\
2621 \\
\end{array}$ & 의 & 5.74 & 44 & 464 & & 0 & 76091 & 105900.6 \\
\hline $4 / 13 / 95$ & \begin{tabular}{r|}
11.3 \\
16.02
\end{tabular} & 699 & $\begin{array}{l}131792 \\
131792\end{array}$ & $\begin{array}{r}2114 \\
0\end{array}$ & 2621 & ut & $0 . \overline{11}$ & 43 & 570 & 2.20 & 20326.22 & 75654 & 135228 a \\
\hline $4 / 14 / 95$ & $\begin{array}{l}16.02 \\
22.15 \\
\end{array}$ & 1277 & $\frac{131792}{131850}$ & 58 & $\begin{array}{l}2621 \\
2621 \\
\end{array}$ & 0 & 5.7 & 43 & 578 & 0.06 & 4887.72 & 77239 & 140114.6 \\
\hline $4 / 15 / 95$ & $\begin{array}{l}22.15 \\
22.15 \\
\end{array}$ & $\begin{array}{l}2383 \\
2383 \\
\end{array}$ & $\begin{array}{r}131850 \\
131850\end{array}$ & 0 & $\begin{array}{l}2621 \\
2621 \\
\end{array}$ & 0 & 5.7 & 43 & 1164 & 0.08 & 6516.96 & 78403 & 146631.6 \\
\hline $4 / 16 / 95$ & \begin{tabular}{r|}
22.15 \\
22.15
\end{tabular} & $\begin{array}{l}2383 \\
2383 \\
\end{array}$ & $\begin{array}{l}131850 \\
131850\end{array}$ & of & $\begin{array}{l}2621 \\
2621 \\
\end{array}$ & 0 & 5.7 & 43 & 0 & & 0 & 78403 & 146631.6 \\
\hline $4 / 17 / 95$ & $\begin{array}{l}22.15 \\
22.15 \\
\end{array}$ & $\begin{array}{l}2383 \\
2383 \\
\end{array}$ & $\begin{array}{l}131850 \\
131850\end{array}$ & 0 & \begin{tabular}{l|}
2621 \\
2621 \\
\end{tabular} & 0 & 5.7 & 43 & 0 & & 0 & 78403 & 146631.6 \\
\hline $4 / 18 / 95$ & 13.16 & $\begin{array}{r}2383 \\
898 \\
\end{array}$ & $\begin{array}{l}131850 \\
136225 \\
\end{array}$ & 4375 & $\begin{array}{r}2621 \\
2621 \\
\end{array}$ & 0 & 5.7 & 43 & 0 & & 0 & 78403 & 146631.6 \\
\hline $4 / 19 / 95$ & 16.59 & $\begin{array}{r}898 \\
1356 \\
\end{array}$ & $\begin{array}{l}136225 \\
136250\end{array}$ & 25 & $\begin{array}{l}2621 \\
2621 \\
\end{array}$ & 0 & 5.64 & 41 & 2888 & & 0 & 81291 & 146631.6 \\
\hline $4 / 20 / 95$ & 12.89 & $\begin{array}{r}1356 \\
867\end{array}$ & $\begin{array}{l}136250 \\
137274 \\
\end{array}$ & 1024 & $\begin{array}{l}2621 \\
2621 \\
\end{array}$ & 0 & 5.65 & 41 & 483 & & 0 & 81774 & 146631.6 \\
\hline $4 / 21 / 95$ & 17.12 & $\begin{array}{r}867 \\
1431 \\
\end{array}$ & 137275 & 1 & 2621 & 0 & 5.68 & 42 & 536 & 0.14 & 11404.68 & 82310 & 158036.3 \\
\hline $4 / 22 / 95$ & 17.12 & 1431 & 137275 & 0 & $\begin{array}{r}2621 \\
2621 \\
\end{array}$ & 0 & 5.63 & 41 & 564 & & 0 & 82874 & 158036.3 \\
\hline $4 / 23 / 95$ & 17.12 & 1431 & 137275 & 0 & $\begin{array}{r}2621 \\
2621 \\
\end{array}$ & 0 & 5.63 & 41 & 0 & & 0 & 82874 & 158036.3 \\
\hline $4 / 24 / 95$ & 23.69 & 2802 & 137276 & 1 & $\begin{array}{l}2621 \\
2621 \\
\end{array}$ & 0 & 5.63 & 41 & 0 & & 0 & 82874 & 158036.3 \\
\hline $4 / 25 / 95$ & 25.3 & 3208 & 137276 & 0 & $\begin{array}{l}2621 \\
2621 \\
\end{array}$ & $\frac{0}{0}$ & 5.63 & 41 & 1372 & & 0 & 84246 & 158036.3 \\
\hline $4 / 26 / 95$ & 25.3 & 3208 & 137276 & 0 & & 0 & 5.63 & 41 & 406 & & 0 & 84652 & 158036.3 \\
\hline $4 / 27 / 95$ & 27.74 & 4061 & 137276 & 0 & $\begin{array}{l}2621 \\
2621\end{array}$ & 0 & 5.63 & 41 & 0 & & 0 & 84652 & 158036.3 \\
\hline $4 / 28 / 95$ & 27.74 & 4061 & 137276 & 0 & $\begin{array}{l}2621 \\
2621\end{array}$ & 0 & 5.68 & 42 & 854 & 0.34 & 27697.08 & 85506 & 185733.4 \\
\hline $4 / 29 / 95$ & 27.74 & 4061 & 137276 & 0 & $\begin{array}{l}2621 \\
2621 \\
\end{array}$ & $\frac{0}{0}$ & 5.68 & 42 & 0 & 0.47 & 38287.14 & 85506 & 224020.5 \\
\hline $4 / 30 / 95$ & 27.74 & 4061 & 137276 & 0 & $\begin{array}{l}2621 \\
2621 \\
\end{array}$ & 0 & 5.68 & 42 & 0 & & 0 & 85506 & 224020.5 \\
\hline & & & & & & 0 & 5.68 & 42 & 0 & & 0 & 85506 & 224020.5 \\
\hline
\end{tabular}




\begin{tabular}{|c|c|c|c|c|c|c|c|c|c|c|c|c|c|}
\hline \multirow[t]{2}{*}{ DATE } & Pri. Level & Pri. Level & Pri/Sec & Pri Flow & Sec Flow & Sec Flow & Sec Level & Sec Level & Total Daily & Daily Rain & Daily Rain & Tot Accu & Tot Accu \\
\hline & inches & & total flow & daily & & & inches & gallons & Water-gal & inches & gallons & Water-gal & Rain-gal \\
\hline $5 / 1 / 95$ & 33.6 & 6792 & 141400 & 4124 & 2621 & 0 & 5.64 & 41 & 6854 & & 0 & 92360 & 224020.5 \\
\hline $5 / 2 / 95$ & 32.41 & 6161 & 142817 & 1417 & 2621 & 0 & 5.63 & 41 & 786 & & 0 & 93146 & 224020.5 \\
\hline $5 / 3 / 95$ & 25.38 & 3315 & 146604 & 3787 & 2621 & 0 & 5.58 & 40 & 940 & & 0 & 94086 & 224020.5 \\
\hline $5 / 4 / 95$ & 28.01 & 4185 & 146603 & -1 & 2621 & 0 & 5.61 & 40 & 869 & & 0 & 94955 & 224020.5 \\
\hline $5 / 5 / 95$ & 29.98 & 5047 & 146605 & 2 & 2621 & 0 & 5.62 & 41 & 865 & 0.02 & 1629.24 & 95820 & 225649.7 \\
\hline $5 / 6 / 95$ & 29.98 & 5047 & 146605 & 0 & 2621 & 0 & 5.62 & 41 & 0 & & 0 & 95820 & 225649.7 \\
\hline $5 / 7 / 95$ & 29.98 & 5047 & 146605 & 0 & 2621 & 0 & 5.62 & 41 & 0 & 0.05 & 4073.1 & 95820 & 229722.8 \\
\hline $5 / 8 / 95$ & 33.71 & 6849 & 146604 & -1 & 2621 & 0 & 5.59 & 40 & 1800 & 0.01 & 814.62 & 97620 & 230537.5 \\
\hline $5 / 9 / 95$ & 15.37 & 1183 & 153043 & 6439 & 2621 & 0 & 5.59 & 40 & 773 & 0.53 & 43174.86 & 98393 & 273712.3 \\
\hline $5 / 10 / 95$ & 19.55 & 1847 & 153043 & 0 & 2621 & 0 & 5.59 & 40 & 664 & 0.02 & 1629.24 & 99057 & 275341.6 \\
\hline $5 / 11 / 95$ & 19.55 & 1847 & 153043 & 0 & 2621 & 0 & 5.59 & 40 & 0 & 0.1 & 8146.2 & 99057 & 283487.8 \\
\hline $5 / 12 / 95$ & 26.91 & 3782 & 153110 & 67 & 2621 & 0 & 5.61 & 40 & 2002 & & 0 & 101059 & 283487.8 \\
\hline $5 / 13 / 95$ & 26.91 & 3782 & 153110 & 0 & 2621 & 0 & 5.61 & 40 & 0 & 0.05 & 4073.1 & 101059 & 287560.9 \\
\hline $5 / 14 / 95$ & 26.91 & 3782 & 153110 & 0 & 2621 & 0 & 5.61 & 40 & 0 & & 0 & 101059 & 287560.9 \\
\hline $5 / 15 / 95$ & 28.01 & 4185 & 155058 & 1948 & 2621 & 0 & 5.59 & 40 & 2351 & & 0 & 103410 & 287560.9 \\
\hline $5 / 16 / 95$ & 29.79 & 4948 & 155058 & 0 & 2621 & 0 & 5.61 & 40 & 763 & & 0 & 104173 & 287560.9 \\
\hline $5 / 17 / 95$ & 29.79 & 4948 & 155058 & 0 & 2621 & 0 & 5.61 & 40 & 0 & & 0 & 104173 & 287560.9 \\
\hline $5 / 18 / 95$ & 32.45 & 6180 & 155058 & 0 & 2621 & 0 & 5.59 & 40 & 1232 & & 0 & 105405 & 287560.9 \\
\hline $5 / 19 / 95$ & 33.37 & 6674 & 155058 & 0. & 2621 & 0 & 5.58 & 40 & 494 & & 0 & 105899 & 287560.9 \\
\hline $5 / 20 / 95$ & 33.37 & 6674 & 155058 & 0 & 2621 & 0 & 5.58 & 40 & 0 & & 0 & 105899 & 287560.9 \\
\hline $5 / 21 / 95$ & 33.37 & 6674 & 155058 & 0 & 2621 & 이 & 5.58 & 40 & 0 & & 0 & 105899 & 287560.9 \\
\hline $5 / 22 / 95$ & 20.21 & 1966 & 161510 & 6452 & 2621 & 0 & 5.58 & 40 & 1744 & & 0 & 107643 & 287560.9 \\
\hline $5 / 23 / 95$ & 22.25 & 2406 & 161510 & 0 & 2621 & 0 & 5.6 & 40 & 440 & & 0 & 108083 & 287560.9 \\
\hline $5 / 24 / 95$ & 24.15 & 2936 & 161510 & 0 & 2621 & 0 & 5.62 & 41 & 531 & & 0 & 108614 & 287560.9 \\
\hline $5 / 25 / 95$ & 25.24 & 3272 & 161510 & 0 & 2621 & 0 & 5.63 & 41 & 336 & 0.01 & 814.62 & 108950 & 288375.5 \\
\hline $5 / 26 / 95$ & 26.29 & 3592 & 161757 & 247 & 2621 & 0 & 5.6 & 40 & 566 & & 0 & 109516 & 288375.5 \\
\hline $5 / 27 / 95$ & 26.29 & 3592 & 161757 & 0 & 2621 & 0 & 5.6 & 40 & 0 & & 0 & 109516 & 288375.5 \\
\hline $5 / 28 / 95$ & 26.29 & 3592 & 161757 & 0 & 2621 & 0 & 5.6 & 40 & 0 & & 0 & 109516 & 288375.5 \\
\hline $5 / 29 / 95$ & 26.29 & 3592 & 161757 & 0 & 2621 & 0 & 5.6 & 40 & 0 & & 0 & 109516 & 288375.5 \\
\hline $5 / 30 / 95$ & 24.18 & 2944 & 164181 & 2424 & 2621 & 0 & 5.64 & 41 & 1777 & & 0 & 111293 & 288375.5 \\
\hline $5 / 31 / 95$ & 24.18 & 2944 & 164181 & 0 & 2621 & 0 & 5.64 & 41 & 0 & & 0 & 111293 & 288375.5 \\
\hline $6 / 1 / 95$ & 10.61 & 625 & 178640 & 14459 & 2621 & 0 & 5.59 & 40 & 12139 & & 0 & 123432 & 288375.5 \\
\hline $6 / 2 / 95$ & 10.61 & 625 & 178640 & 0 & 2621 & 0 & 5.59 & 40 & 0 & & 0 & 123432 & 288375.5 \\
\hline $6 / 3 / 95$ & 10.61 & 625 & 178640 & 0 & 2621 & 0 & 5.59 & 40 & 0 & & 0 & 123432 & 288375.5 \\
\hline $6 / 4 / 95$ & 10.61 & 625 & 178640 & 0 & 2621 & 0 & 5.59 & 40 & 0 & 0.08 & 6516.96 & 123432 & 294892.4 \\
\hline $6 / 5 / 95$ & 22.57 & 2479 & 178640 & 0 & 2621 & 0 & 5.86 & 50 & 1864 & & 0 & 125296 & 294892.4 \\
\hline $6 / 6 / 95$ & 10.69 & 632 & 180878 & 2238 & 2621 & 0 & 5.85 & 50 & 391 & 0.3 & 24438.6 & 125687 & 319331 \\
\hline $6 / 7 / 95$ & 10.69 & 632 & 180878 & 0 & 2621 & 0 & 5.85 & 50 & 0 & & 0 & 125687 & 319331 \\
\hline $6 / 8 / 95$ & 10.69 & 632 & 180878 & 0 & 2621 & 0 & 5.85 & 50 & 0 & & 0 & 125687 & 319331 \\
\hline
\end{tabular}




\begin{tabular}{|c|c|c|c|c|c|c|c|c|c|c|c|c|c|}
\hline DATE & Pri. Level & Pri. Level & Pri/Sec & Pri Flow & Sec Flow & Sec Flow & Sec Level & Sec Level & Total Daily & Daily Rain & Daily Rain & Tot Accu & Tot Accu \\
\hline & inches & gallons & total flow & daily & total & daily & inches & gallons & Water-gal & inches & galions & Water-gal & Rain-gal \\
\hline $6 / 9 / 95$ & 10.69 & 632 & 180878 & 0 & 2621 & 0 & 5.85 & 50 & 0 & & 0 & 125687 & 319331 \\
\hline $6 / 10 / 95$ & 10.69 & 632 & 180878 & 0 & 2621 & 0 & 5.85 & 50 & 0 & & $\underline{0}$ & 125687 & 319331 \\
\hline $6 / 11 / 95$ & 10.69 & 632 & 180878 & 0 & 2621 & 0 & 5.85 & 50 & 0 & & 0 & 125687 & 319331 \\
\hline $6 / 12 / 95$ & 10.69 & 632 & 180878 & 0 & 2621 & 0 & 5.85 & 50 & 0 & & 0 & 125687 & 319331 \\
\hline $6 / 13 / 95$ & 18.5 & 1663 & 182275 & 1397 & 2621 & 0 & 6.13 & 63 & 2441 & & 0 & 128128 & 319331 \\
\hline $6 / 14 / 95$ & 19.98 & 1925 & 182275 & 0 & 2621 & 0 & 6.14 & 63 & 262 & 0.1 & 8146.2 & 128390 & 327477.2 \\
\hline $6 / 15 / 95$ & 21.55 & 2227 & 182275 & 0 & 2621 & 0 & 6.23 & 67 & 306 & 0.01 & 814.62 & 128696 & 328291.9 \\
\hline $6 / 16 / 95$ & 22.78 & 2527 & 182275 & 0 & 2621 & 0 & 6.23 & 67 & 300 & 0.12 & 9775.44 & 128996 & 338067.3 \\
\hline $6 / 17 / 95$ & 22.78 & 2527 & 182275 & 0 & 2621 & 0 & 6.23 & 67 & 0 & 0.04 & 3258.48 & 128996 & 341325.8 \\
\hline $6 / 18 / 95$ & 22.78 & 2527 & 182275 & 0 & 2621 & 0 & 6.23 & 67 & 0 & & 0 & 128996 & 341325.8 \\
\hline $6 / 19 / 95$ & 25.82 & 3449 & 182284 & 0 & 2630 & 9 & 6.2 & 66 & 930 & 0.12 & $\mathbf{9 7 7 5 . 4 4}$ & 129926 & 351101.2 \\
\hline $6 / 20 / 95$ & 25.82 & 3449 & 182284 & 0 & 2630 & 0 & 6.2 & 66 & 0 & & 0 & 129926 & 351101.2 \\
\hline $6 / 21 / 95$ & 25.82 & 3449 & 182284 & 0 & 2630 & 0 & 6.2 & 66 & 0 & & 0 & 129926 & 351101.2 \\
\hline $6 / 22 / 95$ & 25.82 & 3449 & 182284 & 0 & 2630 & 으 & 6.2 & 66 & 0 & & 0 & 129926 & 351101.2 \\
\hline $6 / 23 / 95$ & 25.82 & 3449 & 182284 & 0 & 2630 & 0 & 6.2 & 66 & 0 & & 0 & 129926 & 351101.2 \\
\hline $6 / 24 / 95$ & 25.82 & 3449 & 182284 & 0 & 2630 & 0 & 6.2 & 66 & 0 & & 0 & 129926 & 351101.2 \\
\hline $6 / 25 / 95$ & 25.82 & 3449 & .182284 & 0 & 2630 & 0 & 6.2 & 66 & 0 & & $\underline{0}$ & 129926 & 351101.2 \\
\hline $6 / 26 / 95$ & 18.43 & 1651 & 195812 & 5700 & 10458 & 7828 & 14.08 & 526 & 12190 & & 0 & 142116 & 351101.2 \\
\hline $6 / 27 / 95$ & 19.48 & 1835 & 195812 & 0 & 10458 & 0 & 14.14 & 531 & 189 & & 0 & 142305 & 351101.2 \\
\hline $6 / 28 / 95$ & 19.48 & 1835 & 195812 & 0 & 10458 & 0 & 14.47 & 558 & 27 & & 0 & 142332 & 351101.2 \\
\hline $6 / 29 / 95$ & 22.1 & 2372 & 195812 & 0 & 10458 & 이 & 14.65 & 572 & 551 & & 0 & 142883 & 351101.2 \\
\hline $6 / 30 / 95$ & 22.1 & 2372 & 195812 & 0 & 10458 & 0 & 14.65 & 572 & 0 & & 0 & 142883 & 351101.2 \\
\hline $7 / 1 / 95$ & 22.1 & 2372 & 195812 & 0 & 10458 & 0 & 14.65 & 572 & 0 & 0.02 & 1629.24 & 142883 & 352730.5 \\
\hline $7 / 2 / 95$ & 22.1 & 2372 & 195812 & 0 & 10458 & 0 & 14.65 & 572 & 0 & & 0 & 142883 & 352730.5 \\
\hline $7 / 3 / 95$ & 22.1 & 2372 & 195812 & 0 & 10458 & 0 & 14.65 & 572 & 0 & & 0 & 142883 & 352730.5 \\
\hline $7 / 4 / 95$ & 22.1 & 2372 & 195812 & 0 & 10458 & 인 & 14.65 & 572 & 0 & & 0 & 142883 & 352730.5 \\
\hline $7 / 5 / 95$ & 22.1 & 2372 & 195812 & 0 & 10458 & 0 & 14.65 & 572 & 0 & & 0 & 142883 & 352730.5 \\
\hline $7 / 6 / 95$ & 27.33 & 3930 & 195812 & 0 & 10458 & 0 & 15.27 & 625 & 1611 & 0.02 & 1629.24 & 144494 & 354359.7 \\
\hline $7 / 7 / 95$ & 27.88 & 4106 & 195812 & 0 & 10458 & 0 & 15.29 & 626 & 177 & 0.02 & 1629.24 & 144671 & 355988.9 \\
\hline $7 / 8 / 95$ & 27.88 & 4106 & 195812 & 0 & 10458 & 0 & 15.29 & 626 & 0 & 0.2 & 16292.4 & 144671 & 372281.3 \\
\hline $7 / 9 / 95$ & 27.88 & 4106 & 195812 & 0 & 10458 & 0 & 15.29 & 626 & 0 & 0.04 & 3258.48 & 144671 & 375539.8 \\
\hline $7 / 10 / 95$ & 27.88 & 4106 & 195812 & 0 & 10458 & 0 & 15.29 & 626 & 0 & & 0 & 144671 & 375539.8 \\
\hline $7 / 11 / 95$ & 27.88 & 4106 & 195812 & 0 & 10458 & 0 & 15.29 & 626 & 0 & & 0 & 144671 & 375539.8 \\
\hline $7 / 12 / 95$ & 27.88 & 4106 & 195812 & 0 & 10458 & 0 & 15.29 & 626 & 0 & & 0 & 144671 & 375539.8 \\
\hline $7 / 13 / 95$ & 27.88 & 4106 & 195812 & 0 & 10458 & 0 & 15.29 & 626 & 0 & & 0 & 144671 & 375539.8 \\
\hline $7 / 14 / 95$ & 15.61 & 1215 & 200748 & 4389 & 11005 & 547 & 7.79 & 128 & 1547 & & 0 & 146218 & 375539.8 \\
\hline $7 / 15 / 95$ & 15.61 & 1215 & 200748 & 0 & 11005 & 0 & 7.79 & 128 & of & & 0 & 146218 & 375539.8 \\
\hline $7 / 16 / 95$ & 15.61 & 1215 & 200748 & 0 & 11005 & 0 & 7.79 & 128 & 0 & & 0 & 146218 & 375539.8 \\
\hline $7 / 17 / 95$ & 15.61 & 1215 & 200748 & 0 & 11005 & 0 & 7.79 & 128 & 0 & & 0 & 146218 & 375539.8 \\
\hline
\end{tabular}




\begin{tabular}{|c|c|c|c|c|c|c|c|c|c|c|c|c|c|}
\hline \multirow[t]{2}{*}{ DATE } & \multirow{2}{*}{\begin{tabular}{|l|} 
Pri. Level \\
inches
\end{tabular}} & \multirow{2}{*}{\begin{tabular}{|l|} 
Pri. Level \\
gallons \\
\end{tabular}} & \multirow{2}{*}{ Pri/Sec. } & Priflow & Sec Flow & Sec Flow & Sec Level & \multirow{2}{*}{\begin{tabular}{|l|} 
Sec Level \\
gallons
\end{tabular}} & \multicolumn{2}{|c|}{ Total Daily Daily Rain } & Daily Rain & Tot Accu & Tot Accu \\
\hline & & & & & & & inches & & Water-gal & inches & gallons & Water-gal & Rain-gal \\
\hline $7 / 18 / 95$ & 15.61 & 1215 & 200748 & 0 & 11005 & 0 & 7.79 & 128 & 0 & & 0 & 146218 & 375539.8 \\
\hline $7 / 19 / 95$ & 15.61 & 1215 & 200748 & 0 & 11005 & 0 & 7.79 & 128 & 0 & & 0 & 146218 & 375539.8 \\
\hline $7 / 20 / 95$ & 15.61 & 1215 & 200748 & 0 & 11005 & 0 & 7.79 & 128 & 0 & & 0 & 146218 & 375539.8 \\
\hline $7 / 21 / 95$ & 15.61 & 1215 & 200748 & 0 & 11005 & 0 & 7.79 & 128 & 0 & & 0 & 146218 & 375539.8 \\
\hline $7 / 22 / 95$ & 15.61 & 1215 & 200748 & 0 & 11005 & 0 & 7.79 & 128 & 0 & & 0 & 146218 & 375539.8 \\
\hline $7 / 23 / 95$ & 15.61 & 1215 & 200748 & 0 & 11005 & 0 & 7.79 & 128 & 0 & & 0 & 146218 & 375539.8 \\
\hline $7 / 24 / 95$ & 15.61 & 1215 & 200748 & 0 & 11005 & 0 & 7.79 & 128 & 0 & & 0 & 146218 & 375539.8 \\
\hline $7 / 25 / 95$ & 15.61 & 1215 & 200748 & 0 & 11005 & 0 & 7.79 & 128 & 0 & & 0 & 146218 & 375539.8 \\
\hline $7 / 26 / 95$ & 15.61 & 1215 & 200748 & 0 & 11005 & 0 & 7.79 & 128 & 0 & 0.04 & 3258.48 & 146218 & 378798.3 \\
\hline $7 / 27 / 95$ & 15.61 & 1215 & 200748 & 0 & 11005 & 0 & 7.79 & 128 & 0 & & 0 & 146218 & 378798.3 \\
\hline $7 / 28 / 95$ & 11.6 & 729 & 204000 & 3057 & 11200 & 195 & 7.31 & 109 & 2747 & & 0 & 148965 & 378798.3 \\
\hline $7 / 29 / 95$ & 11.6 & 729 & 204000 & 0 & 11200 & 0 & 7.31 & 109 & 0 & & 0 & 148965 & 378798.3 \\
\hline $7 / 30 / 95$ & 11.6 & 729 & 204000 & 0 & 11200 & 0 & 7.31 & 109 & 0 & & 0 & 148965 & 378798.3 \\
\hline $7 / 31 / 95$ & 11.6 & 729 & 204000 & 0 & 11200 & 0 & 7.31 & 109 & 0 & & 0 & 148965 & 378798.3 \\
\hline $8 / 1 / 95$ & 11.6 & 729 & 204000 & 0 & 11200 & 0 & 7.31 & 109 & 0 & & 0 & 148965 & 378798.3 \\
\hline $8 / 2 / 95$ & 11.6 & 729 & 204000 & 0 & 11200 & 0 & 7.37 & 109 & 0 & & 0 & 148965 & 378798.3 \\
\hline $8 / 3 / 95$ & 11.6 & 729 & 204000 & 0 & 11200 & 0 & 7.31 & 109 & 0 & & 0 & 148965 & 378798.3 \\
\hline $8 / 4 / 95$ & 11.6 & 729 & 204000 & 0 & 11200 & 0 & 7.31 & 109 & 0 & & 0 & 148965 & 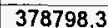 \\
\hline $8 / 5 / 95$ & 11.6 & 729 & 204000 & 0 & 11200 & 0 & 7.31 & 109 & 0 & & 0 & 148965 & 378798.3 \\
\hline $8 / 6 / 95$ & 11.6 & 729 & 204000 & 0 & 11200 & 0 & 7.31 & 109 & 0 & & 0 & 148965 & 378798.3 \\
\hline $8 / 1 / 193$ & 11.0 & $i<y$ & ZưUUUÜ & $\bar{u}_{t}^{\dagger}$ & i izūú & $\bar{v}$ & $7.5 i$ & โธิง & $\hat{v}$ & & $\theta_{1}^{1}$ & i4030 & 570700. \\
\hline $8 / 8 / 95$ & 11.6 & 729 & 204000 & 0 & 11200 & 0 & 7.31 & 109 & 0 & & 0 & 148965 & 378798.3 \\
\hline $8 / 9 / 95$ & 11.6 & 729 & 204000 & 0 & 11200 & 0 & 7.31 & 109 & 0 & & 0 & 148965 & 378798.3 \\
\hline $8 / 10 / 95$ & 11.6 & 729 & 204000 & 01 & 11200 & 0 & 7.31 & 109 & 0 & 0.06 & 4887.72 & 148965 & 383686 \\
\hline $8 / 11 / 95$ & 11.6 & 729 & 204000 & 0 & 11200 & 0 & 7.31 & 109 & 0 & & 0 & 148965 & 383686 \\
\hline $8 / 12 / 95$ & 11.6 & 729 & 204000 & 0 & 11200 & 0 & 7.31 & 109 & 0 & & 0 & 148965 & 383686 \\
\hline $8 / 13 / 95$ & 11.6 & 729 & 204000 & 0 & 11200 & 0 & 7.31 & 109 & 0 & & 0 & 148965 & 383686 \\
\hline $8 / 14 / 95$ & 11.6 & 729 & 204000 & 0 & 11200 & 0 & 7.31 & 109 & 0 & & 0 & 148965 & 383686 \\
\hline $8 / 15 / 95$ & 11.6 & 729 & 204000 & 0 & 11200 & 0 & 7.31 & 109 & 0 & 0.01 & 814.62 & 148965 & 384500.6 \\
\hline $8 / 16 / 95$ & 11.6 & 729 & 204000 & 0 & 11200 & 0 & 7.31 & 109 & 0 & & o) & 148965 & 384500.6 \\
\hline $8 / 17 / 95$ & 11.6 & 729 & 204000 & 0 & 11200 & 0 & 7.31 & 109 & 0 & & 0 & 148965 & 384500.6 \\
\hline $8 / 18 / 95$ & 11.6 & 729 & 204000 & 0 & 11200 & 0 & 7.31 & 109 & 0 & & 0 & 148965 & 384500.6 \\
\hline $8 / 19 / 95$ & 11.6 & 729 & 204000 & 0 & 11200 & 0 & 7.31 & 109 & 0 & & 0 & 148965 & 384500.6 \\
\hline $8 / 20 / 95$ & 11.6 & 729 & 204000 & 0 & 11200 & 0 & 7.31 & 109 & 0 & & 0 & 148965 & 384500.6 \\
\hline $8 / 21 / 95$ & 11.6 & 729 & 204000 & 0 & 11200 & 0 & 7.31 & 109 & 0 & & 0 & 148965 & 384500.6 \\
\hline $8 / 22 / 95$ & 11.6 & 729 & 204000 & 0 & 11200 & 0 & 7.31 & 109 & 0 & & 0 & 148965 & 384500.6 \\
\hline $8 / 23 / 95$ & 11.6 & 729 & 204000 & 0 & 11200 & 0 & 7.31 & 109 & 0] & & 0 & 148965 & 384500.6 \\
\hline $8 / 24 / 95$ & 11.6 & 729 & 204000 & 0] & 11200 & 0 & 7.31 & 109 & 0) & & 0 & 148965 & 384500.6 \\
\hline $8 / 25 / 95$ & 11.6 & 729 & 204000 & 0 & 11200 & 0 & 7.31 & 109 & 0 & & 0 & 148965 & 384500.6 \\
\hline
\end{tabular}




\begin{tabular}{|c|c|c|c|c|c|c|c|c|c|c|c|c|c|}
\hline DATE & Pri. Level & Pri. Level & Pri/sec & Pri Flow & Sec Flow & Sec Flow & Sec Level & Sec Level & Total Daily & Daily Rain & Daily Rain & Tot Accu & Tot Accu \\
\hline & inches & gallons & total flow & daily & & & inches & gallons & Water-gal & & gallons & Water-gal & Rain-gal \\
\hline $8 / 26 / 95$ & 11.6 & 729 & 204000 & 0 & 11200 & 0 & 7.31 & 109 & 0 & & 0 & 148965 & 384500.6 \\
\hline $8 / 27 / 95$ & 11.6 & 729 & 204000 & 0 & 11200 & 0 & 7.31 & 109 & 0 & & 0 & 148965 & 384500.6 \\
\hline $8 / 28 / 95$ & 11.6 & 729 & 204000 & 0 & 11200 & 0 & 7.31 & 109 & 0 & & 0 & 148965 & 384500.6 \\
\hline $8 / 29 / 95$ & 11.6 & 729 & 204000 & 0 & 11200 & 0 & 7.31 & 109 & 0 & & 0 & 148965 & 384500.6 \\
\hline $8 / 30 / 95$ & 11.6 & 729 & 204000 & 0 & 11200 & 0 & 7.31 & 109 & 0 & & 0 & 148965 & 384500.6 \\
\hline $8 / 31 / 95$ & 11.6 & 729 & 204000 & 0 & 11200 & 0 & 7.31 & 109 & 0 & & 0 & 148965 & 384500.6 \\
\hline $9 / 1 / 95$ & 11.6 & 729 & 204000 & 0 & 11200 & 0 & 7.31 & 109 & 0 & & 0 & 148965 & 384500.6 \\
\hline $9 / 2 / 95$ & 11.6 & 729 & 204000 & 0 & 11200 & 0 & 7.31 & 109 & 0 & & 0 & 148965 & 384500.6 \\
\hline $9 / 3 / 95$ & 11.6 & 729 & 204000 & 0 & 11200 & 0 & 7.31 & 109 & 0 & & 0 & 148965 & 384500.6 \\
\hline 9/4/95 & 11.6 & 729 & 204000 & 0 & 11200 & 0 & 7.31 & 109 & 0 & & 0 & 148965 & 384500.6 \\
\hline $9 / 5 / 95$ & 11.6 & 729 & 204000 & 0 & 11200 & 0 & 7.31 & 109 & 0 & & 0 & 148965 & 384500.6 \\
\hline $9 / 6 / 95$ & 11.6 & 729 & 204000 & 0 & 11200 & 0 & 7.31 & 109 & 0 & 0.48 & 39101.76 & 148965 & 423602.4 \\
\hline $9 / 7 / 95$ & 11.6 & 729 & 204000 & 0 & 11200 & 0 & 7.31 & 109 & 0 & 0.19 & 15477.78 & 148965 & 439080.2 \\
\hline $9 / 8 / 95$ & 11.6 & 729 & 204000 & 0 & 11200 & 0 & 7.31 & 109 & 0 & & 0 & 148965 & 439080.2 \\
\hline 9/9/95 & 11.6 & 729 & 204000 & 0 & 11200 & 0 & 7.31 & 109 & 0 & & 0 & 148965 & 439080.2 \\
\hline $9 / 10 / 95$ & 11.6 & 729 & 204000 & 0 & 11200 & 0 & 7.31 & 109 & 0 & & 0 & 148965 & 439080.2 \\
\hline 9/11/95 & 11.6 & 729 & 204000 & 0 & 11200 & 0 & 7.31 & 109 & 0 & & 0 & 148965 & 439080.2 \\
\hline 9/12/95 & 11.6 & 729 & 204000 & 0 & 11200 & 0 & 7.31 & 109 & 0 & & 0 & 148965 & 439080.2 \\
\hline $9 / 13 / 95$ & 11.6 & 729 & 204000 & 0 & 11200 & 0 & 7.31 & 109 & 0 & & 0 & 148965 & 439080.2 \\
\hline $9 / 14 / 95$ & 11.6 & 729 & 204000 & 0 & 11200 & 0 & 7.31 & 109 & 0 & & 0 & 148965 & 439080.2 \\
\hline $9 / 1 \mathrm{~J} / 95$ & 17.6 & $12 y$ & ZUqUOU & v & $11<\mathrm{UU}$ & vi & 1.211 & IUY & ut & & u & 148965 & 439080.2 \\
\hline 9/16/95 & 11.6 & 729 & 204000 & 0 & 11200 & 0. & 7.31 & 109 & 0 & & 0 & 148965 & 439080.2 \\
\hline $9 / 17 / 95$ & 11.6 & 729 & 204000 & 0 & 11200 & 0 & 7.31 & 109 & 0 & & 0 & 148965 & 439080.2 \\
\hline $9 / 18 / 95$ & 11.6 & 729 & 204000 & 0 & 11200 & 0 & 7.31 & 109 & 0 & & 0 & 148965 & 439080.2 \\
\hline 9/19/95 & 11.6 & 729 & 204000 & 0 & 11200 & 0 & 7.31 & 109 & 0 & & 0 & 148965 & 439080.2 \\
\hline $9 / 20 / 95$ & 11.6 & 729 & 204000 & 0 & 11200 & 0 & 7.31 & 109 & 0 & & 0 & 148965 & 439080.2 \\
\hline 9/21/95 & 11.6 & 729 & 204000 & 0 & 11200 & o & 7.31 & 109 & 0 & & 0 & 148965 & 439080.2 \\
\hline $9 / 22 / 95$ & 11.6 & 729 & 204000 & 0 & 11200 & 0 & 7.31 & 109 & 0 & & 0 & 148965 & 439080.2 \\
\hline 9/23/95 & 11.6 & 729 & 204000 & 0 & 11200 & 0 & 7.31 & 109 & 0 & & 0 & 148965 & 439080.2 \\
\hline $9 / 24 / 95$ & 11.6 & 729 & 204000 & 0 & 11200 & 0 & 7.31 & 109 & 0 & & 0 & 148965 & 439080.2 \\
\hline $9 / 25 / 95$ & 11.6 & 729 & 204000 & 0 & 11200 & 0 & 7.31 & 109 & 0 & & 0 & 148965 & $4 \overline{39080.2}$ \\
\hline $9 / 26 / 95$ & 11.6 & 729 & 204000 & 0 & 11200 & 0 & 7.31 & 109 & 0 & & 0 & 148965 & 439080.2 \\
\hline $9 / 27 / 95$ & 11.6 & 729 & 204000 & 0 & 11200 & 0 & 7.31 & 109 & 0 & 0.1 & 8146.2 & 148965 & 447226.4 \\
\hline $9 / 28 / 95$ & 11.6 & 729 & 204000 & 0 & 11200 & 0 & 7.31 & 109 & 0 & & 0 & 148965 & 447226.4 \\
\hline $9 / 29 / 95$ & 11.6 & 729 & 204000 & 0 & 11200 & 0 & 7.31 & 109 & 0 & & 0 & 148965 & 447226.4 \\
\hline $9 / 30 / 95$ & 11.6 & 729 & 204000 & 0 & 11200 & 0 & 7.31 & 109 & 0 & 0.02 & 1629.24 & 148965 & 448855.6 \\
\hline $10 / 1 / 95$ & 11.6 & 729 & 204000 & 0 & 11200 & 0 & 7.31 & 109 & 0 & & 0 & 148965 & 448855.6 \\
\hline $10 / 2 / 95$ & 11.6 & 729 & 204000 & 0 & 11200 & 0 & 7.31 & 109 & 0 & 0.09 & 7331.58 & 148965 & 456187.2 \\
\hline $10 / 3 / 95$ & 23.41 & 2727 & 208187 & 284 & 12103 & 903 & 14.24 & 539 & 6615 & 0.38 & 30955.56 & 155580 & 487142.8 \\
\hline
\end{tabular}




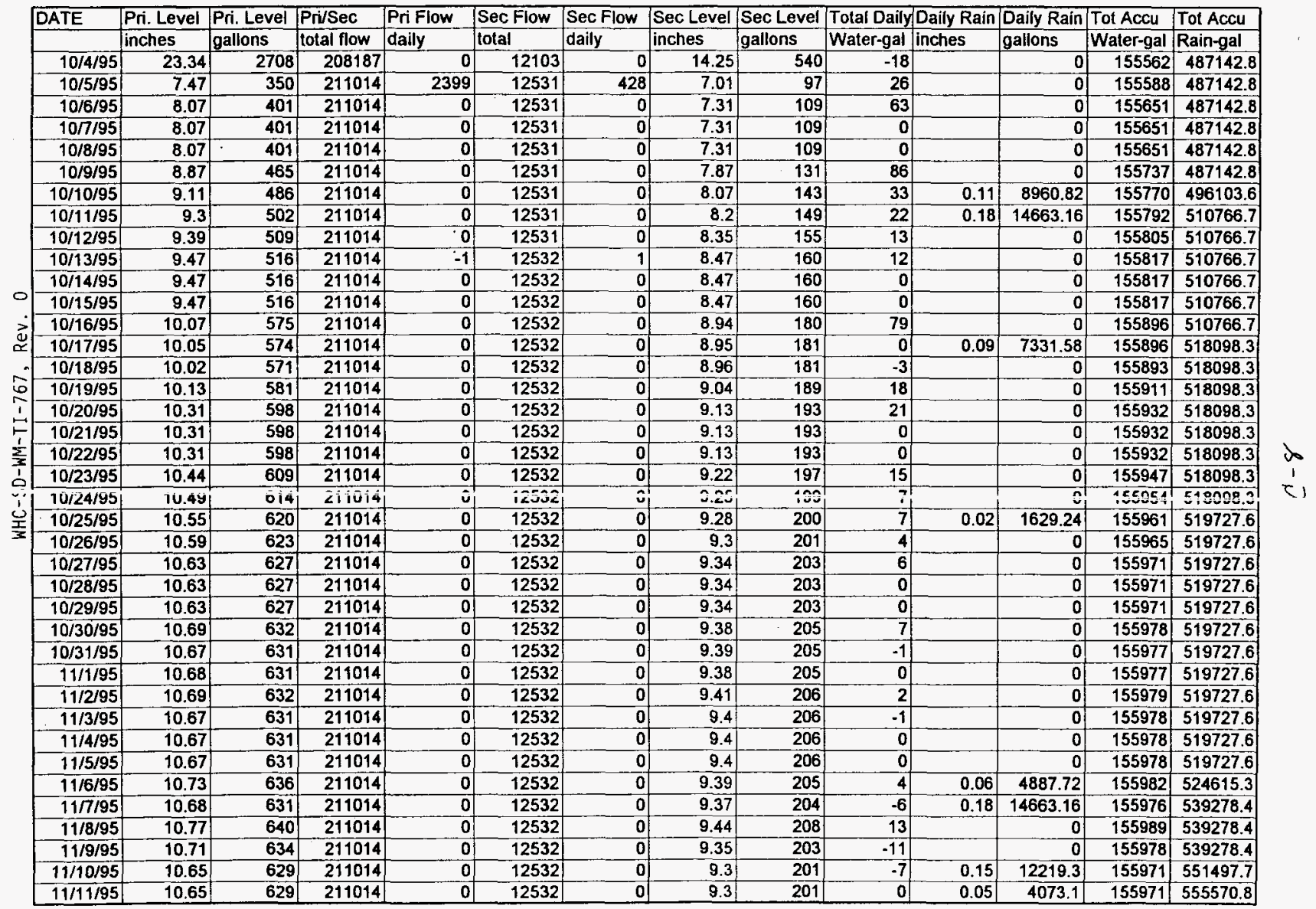




\begin{tabular}{|c|c|c|c|c|c|c|c|c|c|c|c|c|c|}
\hline DATE & Pri. Level & Pri. Level & Pri/Sec & Pri Flow & Sec Flow & Sec Flow & Sec Level & Sec Level & Total Daily & Daily Rain & Daily Rain & Tot Accu & Tot Accu \\
\hline & inches & gallons & total flow & daily & total & daily & inches & gallons & Water-gal & inches & gallons & Water-gal & Rain-gal \\
\hline $11 / 12 / 95$ & $\quad 10.65$ & 629 & 211014 & 0 & 12532 & 0 & \begin{tabular}{|l|l}
9.3 \\
\end{tabular} & 201 & 0 & 0.05 & 4073.1 & 155971 & 559643.9 \\
\hline $11 / 13 / 95$ & 10.72 & 635 & 211014 & 0 & 12532 & 0 & 9.29 & 201 & 6 & 0.15 & 12219.3 & 155977 & 571863.2 \\
\hline $11 / 14 / 95$ & 12.2 & 795 & 211014 & 0 & 12532 & 0 & 9.23 & 198 & 157 & & 0 & 156134 & 571863.2 \\
\hline $11 / 15 / 95$ & 13.53 & 938 & 211014 & 0 & 12532 & 0 & 9.28 & 201 & 146 & 0.01 & 814.62 & 156280 & 572677.9 \\
\hline $11 / 16 / 95$ & 13.95 & 983 & 211014 & 0 & 12532 & 0 & 9.23 & 198 & 42 & & 0 & 156322 & 572677.9 \\
\hline $11 / 17 / 95$ & 14.27 & 1031 & 211014 & 0 & 12532 & 0 & 9.22 & 197 & 47 & 0.02 & 1629.24 & 156369 & $57 \overline{4307.1}$ \\
\hline $11 / 18 / 95$ & 14.27 & 1031 & 211014 & 0 & 12532 & 0 & 9.22 & 197 & 0 & & 0 & 156369 & 574307.1 \\
\hline $11 / 19 / 95$ & 14.27 & 1031 & 211014 & 0 & 12532 & 0 & 9.22 & 197 & 0 & & 0 & 156369 & 574307.1 \\
\hline $11 / 20 / 95$ & 5.15 & 187 & 212100 & 956 & 12662 & 130 & 5.99 & 59 & 104 & & 0 & 156473 & 574307.1 \\
\hline $11 / 21 / 95$ & 6.16 & 255 & 212100 & 0 & 12662 & 0 & 6.27 & 68 & 77 & 0.01 & 814.62 & 156550 & 575121.7 \\
\hline $11 / 22 / 95$ & 6.33 & 266 & 212100 & 0 & 12662 & 0 & 6.27 & 68 & 11 & & 0 & 156561 & 575121.7 \\
\hline $11 / 23 / 95$ & 6.33 & 266 & 212100 & 0 & 12662 & 0 & 6.27 & 68 & 0 & 0.16 & 13033.92 & 156561 & 588155.6 \\
\hline $11 / 24 / 95$ & 6.33 & 266 & 212100 & 0 & 12662 & of & 6.27 & 68 & 0 & 0.01 & 814.62 & 156561 & 588970.3 \\
\hline $\begin{array}{l}11 / 25 / 95 \\
11 / 26 / 95\end{array}$ & 6.33 & 266 & 212100 & 0 & 12662 & 0 & 6.27 & 68 & 0 & & 0 & 156561 & 588970.3 \\
\hline $\begin{array}{l}11 / 26 / 95 \\
11 / 27 / 95\end{array}$ & 6.33 & 266 & 212100 & 0 & 12662 & 0 & 6.27 & 68 & 0 & & 0 & 156561 & 588970.3 \\
\hline $\begin{array}{l}11 / 27 / 95 \\
11 / 28 / 95\end{array}$ & 9.58 & 525 & 212100 & 0 & 12662 & 0 & 6.2 & 66 & 257 & 0.19 & 15477.78 & 156818 & 604448 \\
\hline $\begin{array}{l}11 / 28 / 95 \\
11 / 29 / 95\end{array}$ & 11.3 & 699 & 212100 & 0 & 12662 & 0 & 6.23 & 67 & 175 & & 0 & 156993 & 604448 \\
\hline $\begin{array}{l}11 / 29 / 95 \\
11 / 30 / 95\end{array}$ & 13.09 & 882 & 212100 & 0 & 12662 & 0 & 6.22 & 66 & 182 & & 0 & 157175 & 604448 \\
\hline $\begin{array}{r}11 / 30 / 95 \\
12 / 1 / 95\end{array}$ & 14.3 & 1034 & 212100 & 0 & 12662 & 0 & 6.23 & 67 & 153 & & 0 & 157328 & 604448 \\
\hline $\begin{array}{l}12 / 1 / 95 \\
12 / 2 / 95 \\
\end{array}$ & 14.99 & 1132 & 212100 & 0 & 12662 & 0 & 6.2 & 67 & 98 & & 0 & 157426 & 604448 \\
\hline $\begin{array}{l}12 / 2 / 95 \\
12 / 3 / 95 \\
\end{array}$ & $\begin{array}{l}14.99 \\
14.99\end{array}$ & $\frac{1132}{1132}$ & $\begin{array}{r}212100 \\
212100\end{array}$ & 0 & 12662 & 0 & 0.4 & or & vi & & $\hat{\imath}$ & 157426 & 501410 \\
\hline $\begin{array}{l}12 / 3 / 95 \\
12 / 4 / 95 \\
\end{array}$ & $\begin{array}{l}14.99 \\
15.92 \\
\end{array}$ & $\frac{1132}{1257}$ & $\begin{array}{l}212100 \\
212100\end{array}$ & 0 & 12662 & 0 & 6.2 & 67 & 0 & 0.19 & 15477.78 & 157426 & 619925.8 \\
\hline $\begin{array}{l}12 / 4 / 95 \\
12 / 5 / 95 \\
\end{array}$ & $\frac{15.92}{16.76}$ & $\begin{array}{l}1231 \\
1379\end{array}$ & $\begin{array}{l}212100 \\
212100 \\
\end{array}$ & 0 & 12662 & 0 & 6.15 & 64 & 122 & & 0 & 157548 & 619925.8 \\
\hline $12 / 6 / 95$ & $\begin{array}{r}10.10 \\
17.6\end{array}$ & 1499 & $\begin{array}{l}212100 \\
212100\end{array}$ & 0 & 12662 & 0 & 6.14 & 64 & 122 & & 0 & 157670 & 619925.8 \\
\hline $127 / 95$ & 17.55 & 1492 & $\begin{array}{l}212100 \\
212100\end{array}$ & 0 & 12662 & 0 & 6.09 & 62 & 118 & & 0 & 157788 & 619925.8 \\
\hline $12 / 8 / 95$ & 17.66 & 1508 & $\begin{array}{l}212100 \\
212100\end{array}$ & 0 & 12662 & 0 & 6.12 & 63 & -6 & & 0 & 157782 & 619925.8 \\
\hline $12 / 9 / 95$ & 17.66 & 1508 & $\begin{array}{l}212100 \\
212100\end{array}$ & 0 & 12662 & 0 & 6.09 & 62 & 15 & & 0 & 157797 & 619925.8 \\
\hline $12 / 10 / 95$ & 17.66 & 1508 & $\begin{array}{l}212100 \\
212100\end{array}$ & 0 & 12662 & 0 & 6.09 & 62 & 0 & 0.13 & 10590.06 & 157797 & 630515.9 \\
\hline $12 / 11 / 95$ & 17.93 & 1546 & $\begin{array}{l}212100 \\
212100\end{array}$ & 0 & 12662 & 0 & 6.09 & 62 & 0 & 0.38 & 30955.56 & 157797 & 661471.4 \\
\hline $12 / 12 / 95$ & 25.01 & 3200 & $\begin{array}{l}212100 \\
212100\end{array}$ & 0 & 12662 & 0 & 6.12 & 63 & 39 & 0.4 & 32584.8 & 157836 & 694056.2 \\
\hline $12 / 13 / 95$ & 33.82 & 6905 & $\begin{array}{l}212100 \\
215345 \\
\end{array}$ & 0 & 12662 & 0 & 6.14 & 64 & 1655 & 0.65 & 52950.3 & 159491 & 747006.5 \\
\hline $12 / 14 / 95$ & 20.63 & 2041 & \begin{tabular}{|l|}
215345 \\
222123 \\
\end{tabular} & 3245 & 12662 & 0 & 6.05 & 61 & 6947 & & 0 & 166438 & 747006.5 \\
\hline $12 / 15 / 95$ & 17.08 & 1425 & $\begin{array}{l}222123 \\
224180 \\
\end{array}$ & 6778 & 12662 & 0 & 5.99 & 59 & 1912 & 0.02 & 1629.24 & 168350 & 748635.8 \\
\hline $12 / 16 / 95$ & 17.08 & 1425 & $\begin{array}{l}224180 \\
224180\end{array}$ & 2057 & 12662 & 0 & 5.98 & 58 & 1440 & & 0 & 169790 & 748635.8 \\
\hline $12 / 17 / 95$ & 17.08 & 1425 & $\begin{array}{l}224180 \\
224180\end{array}$ & 0 & 12662 & 0 & 5.98 & 58 & 0 & & 0 & 169790 & 748635.8 \\
\hline $12 / 18 / 95$ & 27.4 & 3952 & $\begin{array}{l}224180 \\
224181 \\
\end{array}$ & 0 & 12662 & 0 & 5.98 & 58 & 0 & & 0 & 169790 & 748635.8 \\
\hline $12 / 19 / 95$ & 14.05 & 1005 & $\begin{array}{l}224181 \\
227979 \\
\end{array}$ & $\begin{array}{r}1 \\
3798\end{array}$ & 12662 & 0 & 5.91 & 53 & 2523 & & 0 & 172313 & 748635.8 \\
\hline $12 / 20 / 95$ & 18.79 & $17 \overline{11}$ & $\begin{array}{l}227979 \\
227979 \\
\end{array}$ & $\begin{array}{r}3798 \\
0\end{array}$ & 12662 & 0 & 5.88 & 52 & 850 & 0.01 & 814.62 & 173163 & 749450.4 \\
\hline & & & & 0 & 12662 & 0 & 5.83 & 50 & 704 & & 0 & 173867 & 749450.4 \\
\hline
\end{tabular}




\begin{tabular}{|c|c|c|c|c|c|c|c|c|c|c|c|c|c|}
\hline DATE & Pri. Level & Pri. Level & Pri/Sec & Pri Flow & Sec Flow & Sec Flow & Sec Level & \begin{tabular}{|l|} 
Sec Level \\
\end{tabular} & Total Daily & Daily Rain & \begin{tabular}{|l} 
Daily Rain \\
\end{tabular} & Tot Accu & \begin{tabular}{|l|} 
Tot Accu \\
Rain-gal
\end{tabular} \\
\hline & inches & gallons & total flow & daily & & & & & & & & Water-gal & \\
\hline $12 / 21 / 95$ & 18.79 & 1711 & 227979 & 0 & 12662 & 0 & 5.83 & 50 & 0 & & 0 & 173867 & \begin{tabular}{|l|}
749450.4 \\
\end{tabular} \\
\hline $12 / 22 / 95$ & 18.79 & 1711 & 227979 & 0 & 12662 & 0 & 5.83 & 50 & 0 & & 0 & 173867 & 749450.4 \\
\hline $12 / 23 / 95$ & 18.79 & 1711 & 227979 & 0 & 12662 & 0 & 5.83 & 50 & 0 & & 0 & 173867 & 749450.4 \\
\hline $12 / 24 / 95$ & 18.79 & 1711 & 227979 & 0 & 12662 & 0 & 5.83 & 50 & 0 & & 0 & 173867 & 749450.4 \\
\hline $12 / 25 / 95$ & 18.79 & 1711 & 227979 & 0 & 12662 & 0 & 5.83 & 50 & 0 & & 0 & 173867 & 749450.4 \\
\hline $12 / 26 / 95$ & 18.79 & 1711 & 227979 & 0 & 12662 & 0 & 5.83 & 50 & 0 & & 0 & 173867 & 749450.4 \\
\hline $12 / 27 / 95$ & 31.35 & 5665 & 227979 & 0 & 12662 & 0 & 5.72 & 46 & 3950 & & 0 & 177817 & 749450.4 \\
\hline $12 / 28 / 95$ & 13.03 & 884 & 233517 & 5538 & 12662 & 0 & 5.67 & 44 & 755 & 0.04 & 3258.48 & 178572 & 752708.9 \\
\hline $12 / 29 / 95$ & 16.6 & 1357 & 233517 & 0 & 12662 & 0 & 5.66 & 44 & 473 & 0.22 & 17921.64 & 179045 & 770630.5 \\
\hline $12 / 30 / 95$ & 16.6 & 1357 & 233517 & 0 & 12662 & 0 & 5.66 & 44 & 0 & 0.28 & 22809.36 & 179045 & 793439.9 \\
\hline $12 / 31 / 95$ & 16.6 & 1357 & 233517 & 0 & 12662 & 0 & 5.66 & 44 & 0 & & 0 & 179045 & 793439.9 \\
\hline $1 / 1 / 96$ & 16.6 & 1357 & 233517 & 0) & 12662 & 0 & 5.66 & 44 & 0 & 0.02 & 1629.24 & 179045 & 795069.1 \\
\hline $1 / 2 / 96$ & 36.3 & 8460 & 233517 & 0 & 12662 & 0. & 5.64 & 43 & 7102 & & $\mathbf{0}$ & 186147 & 795069.1 \\
\hline $1 / 3 / 96$ & 21.66 & 2372 & 242240 & 8723 & 12662 & 0 & 5.68 & 45 & 2637 & & 0 & 188784 & 795069.1 \\
\hline $1 / 4 / 96$ & 26.97 & 3910 & 242240 & 0 & 12662 & 0 & 5.58 & 40 & 1533 & & 0 & 190317 & 795069.1 \\
\hline $1 / 5 / 96$ & 29.57 & 4870 & 242240 & 0 & 12662 & 0 & 5.59 & 41. & 961 & 0.15 & 12219.3 & 191278 & 807288.4 \\
\hline $1 / 6 / 96$ & 29.57 & 4870 & 242240 & 0 & 12662 & 0 & 5.59 & 41 & 0 & 0.01 & 814.62 & 191278 & 808103 \\
\hline $1 / 7 / 96$ & 29.57 & 4870 & 242240 & 0 & 12662 & 0 & 5.59 & 41 & 0 & 0.1 & 8146.2 & 191278 & 816249.2 \\
\hline $1 / 8 / 96$ & 28.95 & 4670 & 247280 & 5040 & 12662 & 0 & 5.51 & 38 & 4837 & & 0 & 196115 & 816249.2 \\
\hline $1 / 9 / 96$ & 32.69 & 6410 & 247290 & 10 & 12662 & 0 & 5.48 & 38 & 1750 & 0.04 & 3258.48 & 197865 & 819507.7 \\
\hline $1 / 10 / 96$ & 27.93 & $244 \bar{U}$ & 253515 & $0<20$ & $1200<$ & ut & $3.5<$ & so & 2250 & & 0 & $20 \bar{i} i 2 i$ & $\overline{0}$ i950̄ \\
\hline $1 / 11 / 96$ & 20.4 & 2100 & 255757 & 2241 & 12662 & 0 & 5.49 & 38 & 1901 & & 0 & 202022 & 819507.7 \\
\hline $1 / 12 / 96$ & 26.32 & 3630 & 255757 & 0 & 12662 & 0 & 5.49 & 38 & 1530 & & 0 & 203552 & 819507.7 \\
\hline $1 / 13 / 96$ & 26.32 & 3630 & 255757 & 0 & 12662 & 0 & 5.49 & 38 & 0 & & 0 & 203552 & 819507.7 \\
\hline $1 / 14 / 96$ & 26.32 & 3630 & 255757 & 0 & 12662 & 0 & 5.49 & 38 & 0 & 0.02 & 1629.24 & 203552 & 821137 \\
\hline $1 / 15 / 96$ & 28.54 & 4490 & 259360 & 3603 & 12662 & 0 & 5.39 & 35 & 4460 & 0.02 & 1629.24 & 208012 & 822766.2 \\
\hline $1 / 16 / 96$ & 29.29 & 4790 & 259700 & 340 & 12662 & 0 & 5.37 & 34 & 639 & & 0 & 208651 & 822766.2 \\
\hline $1 / 17 / 96$ & 13.68 & 870 & 265590 & 5890 & 12662 & 0 & 5.38 & 35 & 1971 & & 0 & 210622 & 822766.2 \\
\hline $1 / 18 / 96$ & 16.15 & 1245 & 266157 & 567 & 12662 & 0 & 5.34 & 34 & 941 & 0.28 & 22809.36 & 211563 & 845575.6 \\
\hline $1 / 19 / 96$ & 16.7 & 1325 & 267200 & 1043 & 12662 & 0 & 5.34 & 34 & 1123 & 0.01 & 814.62 & 212686 & 846390.2 \\
\hline $1 / 20 / 96$ & 16.7 & 1325 & 267200 & 0 & 12662 & 0 & 5.34 & 34 & 0 & 0.25 & 20365.5 & 212686 & 866755.7 \\
\hline $1 / 21 / 96$ & 16.7 & 1325 & 267200 & 0 & 12662 & 0 & 5.34 & 34 & 0 & & 0 & 212686 & 866755.7 \\
\hline $1 / 22 / 96$ & 24.32 & 3065 & 268334 & 1134 & 12662 & 0 & 5.31 & 33. & 2873 & & 0 & 215559 & 866755.7 \\
\hline $1 / 23 / 96$ & 27.02 & 3920 & 268335 & 1 & 12662 & 0 & 5.26 & 31 & 854 & 0.01 & 814.62 & 216413 & 867570.3 \\
\hline $1 / 24 / 96$ & 16.02 & 1230 & 272041 & 3706 & 12662 & 0 & 5.25 & 31. & 1016 & 0.26 & 21180.12 & 217429 & 888750.4 \\
\hline $1 / 25 / 96$ & 14.33 & 965 & 273165 & 1124 & 12662 & 0 & 5.28 & 32 & 860 & & 0 & 218289 & 888750.4 \\
\hline $1 / 26 / 96$ & 19.71 & 1930 & 273165 & 0 & 12662 & 0 & 5.26 & 31 & 964 & & 0 & 219253 & 888750.4 \\
\hline $1 / 27 / 96$ & 19.71 & 1930 & 273165 & 0 & 12662 & of & 5.26 & 31 & 0 & 0.21 & 17107.02 & 219253 & 905857.4 \\
\hline $1 / 28 / 96$ & 19.71 & 1930 & 273165 & 이 & 12662 & 0 & 5.26 & 31 & 0 & 0.02 & 1629.24 & 219253 & 907486.7 \\
\hline
\end{tabular}


Trench 34

sheet1

\begin{tabular}{|c|c|c|c|c|c|c|c|c|c|c|c|c|c|}
\hline DATE & Pri. Level & Pri. Level & Pri/Sec & Pri Flow & Sec Flow & Sec Flow & Sec Level & Sec Level & Total Daily & Daily Rain & Daily Rain & Tot Accu & Tot Accu \\
\hline & inches & gallons & total flow & daily & total & daily & inches & gallons & Water-gal i & inches & gallons & Water-gal & Rain-gal \\
\hline $1 / 29 / 96$ & 23.38 & 2790 & 274913 & \begin{tabular}{|l|}
1748 \\
2539 \\
\end{tabular} & 12662 & 0 & 5.24 & 31 & 2608 & 0.02 & 1629.24 & 221861 & 909115.9 \\
\hline $1 / 30 / 96$ & 14.24 & 950 & 277452 & $\begin{array}{r}2539 \\
0\end{array}$ & 12662 & 0 & 5.2 & 30 & 698 & & 0 & 222559 & 909115.9 \\
\hline $1 / 31 / 96$ & 19.01 & 1796 & 277452 & $\begin{array}{l}0 \\
0\end{array}$ & 12662 & 0 & 5.1 & 28 & 844 & & 0 & 223403 & 909115.9 \\
\hline $2 / 1 / 96$ & 21.22 & 2263 & 277452 & 0 & 12662 & 0 & 5.19 & 30 & 469 & & 0 & 223872 & 909115.9 \\
\hline 2/2/96 & 22.89 & 2650 & 277452 & 0 & 12662 & 0 & 5.17 & 29 & 386 & & 0 & 224258 & 909115.9 \\
\hline 2/3/96 & 22.89 & 2650 & 277452 & 0 & 12662 & 0 & 5.17 & 29 & 0 & 0.09 & 7331.58 & 224258 & 916447.5 \\
\hline $2 / 4 / 96$ & 22.89 & 2650 & 277452 & 0 & 12662 & 0 & 5.17 & 29 & 0 & 0.06 & 4887.72 & 224258 & 921335.2 \\
\hline $2 / 5 / 96$ & 9.24 & 350 & 280615 & 3163 & 12662 & 0 & 5.14 & 28 & 862 & 0.1 & 8146.2 & 225120 & 929481.4 \\
\hline $2 / 6 / 96$ & 11.42 & 590 & 280615 & 0 & 12662 & 0 & 5.1 & 28 & 240 & 0.09 & 7331.58 & 225360 & 936813 \\
\hline $2 / 7 / 96$ & 30.1 & 5081 & 280740 & 125 & 12662 & 0 & 5.05 & 26 & 4614 & 0.29 & 23623.98 & 229974 & 960437 \\
\hline $\begin{array}{l}2 / 8 / 96 \\
2 / 9 / 96 \\
\end{array}$ & 22.29 & 2510 & 297864 & 17124 & 12662 & 0 & 5.34 & 34 & 14561 & 0.01 & 814.62 & 244535 & 961251.6 \\
\hline $\begin{array}{r}2 / 9 / 96 \\
2 / 10 / 96\end{array}$ & 16.01 & 1226 & 310482 & 12618 & 12662 & 0 & 5.38 & 35 & 11335 & & - & 255870 & 961251.6 \\
\hline $\begin{array}{l}2 / 10 / 96 \\
2 / 11 / 96 \\
\end{array}$ & 16.01 & 1226 & 310482 & 0 & 12662 & 0 & 5.38 & 35 & 0 & & of & 255870 & 961251.6 \\
\hline $\begin{array}{l}2 / 11 / 96 \\
2 / 12 / 96\end{array}$ & 16.01 & 1226 & 310482 & 0 & 12662 & 0 & 5.38 & 35 & 0 & & 0 & 255870 & 961251.6 \\
\hline $\begin{array}{l}2 / 12 / 96 \\
2 / 13 / 96 \\
\end{array}$ & 28.56 & 4450 & 326465 & 15983 & 12662 & 0 & 5.7 & 45 & 19217 & & 0 & 275087 & 961251.6 \\
\hline $\begin{array}{r}2 / 13 / 96 \\
2 / 14 / 96\end{array}$ & 28.87 & 4650 & 331922 & 5457 & 12662 & 0 & 5.8 & 49 & 5661 & & 0 & 280748 & 961251.6 \\
\hline $\begin{array}{l}/ 14 / 96 \\
2 / 15 / 96\end{array}$ & 28.32 & 4395 & 337510 & 5588 & 12662 & 0 & 5.88 & 51 & 5335 & & 0 & 286083 & 961251.6 \\
\hline $\begin{array}{l}2 / 15 / 96 \\
2 / 16 / 96\end{array}$ & 26.8 & 3900 & 342563 & 5053 & 12662 & 0 & 5.96 & 57 & 4564 & & 0 & 290647 & 961251.6 \\
\hline \begin{tabular}{|c|}
$2 / 16 / 96$ \\
$/ 17 / 96$
\end{tabular} & 25.53 & 3410 & 347165 & 4602 & 12662 & 0 & 6.01 & 59 & 4114 & & 0 & 294761 & 961251.6 \\
\hline \begin{tabular}{|l|}
$3 / 17 / 96$ \\
$2 / 18 / 96$
\end{tabular} & 25.53 & 3410 & 347165 & 0 & 12662 & 0 & 6.01 & 59 & 0 & 0.11 & 8960.82 & 294761 & 970212.4 \\
\hline \begin{tabular}{|l|}
$2 / 18 / 96$ \\
$2 / 19 / 96$
\end{tabular} & 25.53 & 3410 & 347165 & 0 & 12662 & $u+$ & $0 . \overline{1}$ & 53 & 3 & 0.02 & 214295 & 204761 & 9736563 \\
\hline $\begin{array}{l}2 / 19 / 96 \\
2 / 20 / 96\end{array}$ & 25.53 & 3410 & 347165 & 0 & 12662 & 0 & 6.01 & 59 & 0 & & 0 & 294761 & 972656.3 \\
\hline $\begin{array}{l}2 / 20 / 96 \\
2 / 21 / 96\end{array}$ & 24.03 & 2980 & 362986 & 15821 & 12662 & 0 & 6.23 & 66 & 15398 & 0.05 & 4073.1 & 310159 & 976729.4 \\
\hline $\begin{array}{l}2 / 21 / 96 \\
2 / 22 / 96 \\
\end{array}$ & 23.12 & 2720 & 366191 & 3205 & 12662 & 0 & 6.31 & 70 & 2949 & 0.03 & 2443.86 & 313108 & 979173.2 \\
\hline \begin{tabular}{l|}
$2 / 22 / 96$ \\
$2 / 23 / 96$ \\
\end{tabular} & 23.35 & 2775 & 368745 & 2554 & 12662 & 의 & 6.28 & 69 & 2608 & & 0 & 315716 & 979173.2 \\
\hline $\begin{array}{l}2 / 23 / 96 \\
2 / 24 / 96\end{array}$ & 19.47 & 1890 & 372020 & 3275 & 12662 & of & 6.37 & 72 & 2393 & 0.01 & 814.62 & 318109 & 979987.9 \\
\hline $\begin{array}{l}2 / 24 / 96 \\
2 / 25 / 96\end{array}$ & 19.47 & 1890 & 372020 & 0 & 12662 & 0 & 6.37 & 72 & 0 & 0.13 & 10590.06 & 318109 & 990577.9 \\
\hline $2 / 25 / 96$ & $19 . \overline{47}$ & 1890 & 372020 & 0 & 12662 & of & 6.37 & 72 & 0 & 0.22 & 17921.64 & 318109 & 1008500 \\
\hline 2/26/96 & 28.82 & 4600 & 376401 & 4381 & 12662 & 0 & 6.34 & 71 & 7090 & & 0 & 325199 & 1008500 \\
\hline $2 / 27 / 96$ & 24.55 & 3150 & 381440 & 5039 & 12662 & 0 & 6.35 & 71 & 3589 & & 0 & 328788 & 1008500 \\
\hline $2 / 28 / 96$ & 23.2 & 2750 & 386505 & 5065 & 12662 & 0 & 6.34 & 71 & 4665 & & 0 & 333453 & 1008500 \\
\hline $2 / 29 / 96$ & 22.28 & 2500 & 387505 & 999 & 12663 & 1 & 6.27 & 70 & 749 & & 0 & 334202 & 1008500 \\
\hline $3 / 1 / 96$ & 25.35 & 3250 & 387665 & 160 & 12663 & 0 & 6.31 & 70 & 910 & & 0 & 335112 & 1008500 \\
\hline $3 / 2 / 96$ & 25.35 & 3250 & 387665 & 0 & 12663 & 0 & 6.31 & 70 & 0 & & 0 & 335112 & 1008500 \\
\hline $3 / 3 / 96$ & 25.35 & 3250 & 387665 & 0 & 12663 & 0 & 6.31 & 70 & 0 & 0.01 & 814.62 & 335112 & 1009314 \\
\hline $3 / 4 / 96$ & 22.6 & 2570 & 395720 & 8055 & 12663 & 0 & 6.26 & 68 & 7373 & 0.3 & 24438.6 & 342485 & 1033753 \\
\hline $3 / 5 / 96$ & 25.33 & 3350 & 397820 & 2100 & 12663 & 0 & 6.29 & 69 & 2881 & & 0 & 345366 & 1033753 \\
\hline $3 / 6 / 96$ & 27.34 & 4020 & 401141 & 3321 & 12663 & 0 & 6.28 & 69 & 3991 & & 0 & 349357 & 1033753 \\
\hline $3 / 7 / 96$ & 23.34 & 2800 & 405150 & 4009 & 12663 & 0 & 6.28 & 69 & 2789 & 0.1 & 8146.2 & 352146 & 1041899 \\
\hline
\end{tabular}




\begin{tabular}{|c|c|c|c|c|c|c|c|c|c|c|c|c|c|}
\hline DATE & Pri. Level & Pri. Level & Pri/Sec & \begin{tabular}{|l} 
Pri Flow \\
daily
\end{tabular} & Sec Flow & Sec Flow & \begin{tabular}{|l|} 
Sec Level \\
inches
\end{tabular} & \begin{tabular}{|l|} 
Sec Level \\
gallons
\end{tabular} & \begin{tabular}{|l|} 
Total Daily \\
Water-gal
\end{tabular} & \begin{tabular}{|l|} 
Daily Rain \\
inches \\
\end{tabular} & \begin{tabular}{|l|} 
Daily Rain \\
gallons \\
\end{tabular} & \begin{tabular}{|l|} 
Tot Accu \\
Water-aa
\end{tabular} & \begin{tabular}{|l|} 
Tot Accu \\
Rain-gal
\end{tabular} \\
\hline & inches & gallons & \begin{tabular}{|r|} 
total flow \\
407940
\end{tabular} & & & & inches & gallons & Water-gal & & & | water-gai & \\
\hline $3 / 8 / 96$ & 21.92 & 2440 & 407940 & \begin{tabular}{|r|}
2790 \\
\end{tabular} & 12663 & 0 & 6.25 & 67 & 2428 & & 0 & 354574 & 1041899 \\
\hline $3 / 9 / 96$ & 21.92 & 2440 & 407940 & 0 & 12663 & 0 & 6.25 & 67 & 0 & & 0 & 354574 & 1041899 \\
\hline $3 / 10 / 96$ & 29.75 & 5000 & 407940 & 0 & 12663 & 0 & 6.22 & 66 & 2559 & & 0 & 357133 & 1041899 \\
\hline $3 / 11 / 96$ & 22.19 & 2450 & 415065 & 7125 & 12663 & 0 & 6.23 & 67 & 4576 & 0.03 & 2443.86 & 361709 & 1044343 \\
\hline $3 / 12 / 96$ & 19.65 & 1900 & 417493 & 2428 & 12663 & 0 & 6.2 & 66 & 1877 & & 0 & 363586 & 1044343 \\
\hline $3 / 13 / 96$ & 21.14 & 2240 & 419004 & 1511 & 12663 & 0 & 6.2 & 66 & 1851 & & 0 & 365437 & 1044343 \\
\hline $3 / 14 / 96$ & 18.33 & 1660 & 421129 & 2125 & 12663 & 0 & 6.18 & 65 & 1544 & & 0 & 366981 & 1044343 \\
\hline $3 / 15 / 96$ & 16.5 & 1320 & 422912 & 1783 & 12663 & 0 & 6.18 & 65 & 1443 & & 0 & 368424 & 1044343 \\
\hline $3 / 16 / 96$ & 16.5 & 1320 & 422912 & 0 & 12663 & 0 & 6.18 & 65 & 0 & & 0 & 368424 & 1044343 \\
\hline \begin{tabular}{|l|}
$3 / 17 / 96$ \\
$3 / 18 / 96$ \\
\end{tabular} & 16.5 & 1320 & 422912 & 0 & 12663 & 0 & 6.18 & 65 & 0 & & 0 & 368424 & 1044343 \\
\hline \begin{tabular}{|l|}
$3 / 18 / 96$ \\
$3 / 19 / 96$
\end{tabular} & 28.5 & 4480 & 424635 & 1723 & 12663 & 0 & 6.17 & 65 & 4883 & & 0 & 373307 & 1044343 \\
\hline $3 / 19 / 96$ & 16.61 & 1325 & 427835 & 3200 & 12663 & 0 & 6.17 & 65 & 45 & & 0 & 373352 & 1044343 \\
\hline $3 / 20 / 96$ & 22.38 & 2530 & 427835 & 0 & 12663 & 0 & 6.17 & 65 & 1205 & & 0 & 374557 & 1044343 \\
\hline $3 / 21 / 96$ & 15.4 & 1140 & 430746 & 2911 & 12663 & 0 & 6.15 & 64 & 1520 & & 0 & 376077 & 1044343 \\
\hline $3 / 22 / 96$ & 15.17 & 1100 & 431750 & 1004 & 12663 & 0 & 6.2 & 66 & 966 & 0.13 & 10590.06 & 377043 & 1054933 \\
\hline 3/23/96 & 15.17 & 1100 & 431750 & 0 & 12663 & 0 & 6.2 & 66 & 0 & & 0 & 377043 & 1054933 \\
\hline $3 / 24 / 96$ & 15.17 & 1100 & 431750 & 0 & 12663 & 0 & 6.2 & 66 & 0 & & 0 & 377043 & 1054933 \\
\hline $3 / 25 / 96$ & 24.43 & 3075 & 432290 & 540 & 12663 & 0 & 6.12 & 63 & 2512 & & 0 & 379555 & 1054933 \\
\hline $3 / 26 / 96$ & 15.29 & 1115 & 434665 & 2375 & 12663 & 0 & 6.11 & 63 & 415 & & 0 & 379970 & 1054933 \\
\hline $3 / 27 / 96$ & 14.85 & 1015 & 435510 & 845 & 12663 & 0 & 6.15 & 64 & 746 & & 0 & 380716 & 1054933 \\
\hline $3 / 28 / 96$ & 20.3 & 2070 & 435308 & -4 & $1<00 \mathrm{~S}$ & ui & 0.12 & $\hat{0} \mathbf{3}$ & $i \overline{u j 2}$ & & $\hat{v}$ & 301700 & 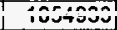 \\
\hline $3 / 29 / 96$ & 22.93 & 2590 & 435600 & 92 & 12663 & 0 & 6.13 & 63 & 612 & & 0 & 382380 & 1054933 \\
\hline $3 / 30 / 96$ & 22.93 & 2590 & 435600 & 0 & 12663 & 0 & 6.13 & 63 & 0 & & 0 & 382380 & 1054933 \\
\hline $3 / 31 / 96$ & 22.93 & 2590 & 435600 & 0 & 12663 & 0 & 6.13 & 63 & 0 & 0.26 & 21180.12 & 382380 & 1076113 \\
\hline $4 / 1 / 96$ & 23.32 & 2675 & 437910 & 2310 & 12663 & 0 & 6.15 & 64 & 2396 & 0.12 & \begin{tabular}{|l|}
9775.44 \\
\end{tabular} & 384776 & 1085888 \\
\hline $4 / 2 / 96$ & 21.25 & 2270 & 440230 & 2320 & 12663 & 0 & 6.18 & 65 & 1916 & & 0 & 386692 & 1085888 \\
\hline $4 / 3 / 96$ & 16.6 & 1320 & 442880 & 2650 & 12663 & 0 & 6.14 & 64 & 1699 & & 0 & 388391 & 1085888 \\
\hline $4 / 4 / 96$ & 15.4 & 1130 & 443620 & 740 & 12663 & 0 & 6.12 & 63 & 549 & & 0 & 388940 & 1085888 \\
\hline $4 / 5 / 96$ & 14.84 & 900 & 444670 & 1050 & 12663 & 0 & 6.11 & 63 & 820 & & 0 & 389760 & 1085888 \\
\hline $4 / 6 / 96$ & 14.84 & 900 & 444670 & 0 & 12663 & 0 & 6.11 & 63 & 0 & & 0 & 389760 & 1085888 \\
\hline $4 / 7 / 96$ & 14.84 & 900 & 444670 & 0 & 12663 & 0 & 6.11 & 63 & 0 & & 0 & 389760 & 1085888 \\
\hline $4 / 8 / 96$ & 22.87 & 2675 & 445557 & 887 & 12663 & 0 & 6.13 & 63 & 2662 & & 0 & 392422 & 1085888 \\
\hline $4 / 9 / 96$ & 18.77 & 1760 & 448050 & 2493 & 12663 & 0 & 6.11 & 63 & 1578 & & 0 & 394000 & 1085888 \\
\hline $4 / 10 / 96$ & 12.9 & 770 & 449350 & 1300 & 12663 & 0 & 6.12 & 63 & 310 & & 0 & 394310 & 1085888 \\
\hline $4 / 11 / 96$ & 12.64 & 710 & 449985 & 635 & 12663 & 0 & 6.11 & 63 & 575 & & 0 & 394885 & 1085888 \\
\hline $4 / 12 / 96$ & 12.64 & 710 & 450675 & 690 & 12663 & 0 & 6.11 & 63 & 690 & & 0 & 395575 & 1085888 \\
\hline 4/13/96 & 12.64 & 710 & 450675 & 0 & 12663 & 0 & 6.11 & 63 & 0 & & 0 & 395575 & 1085888 \\
\hline $4 / 14 / 96$ & 12.64 & 710 & 450675 & 0 & 12663 & 0 & 6.11 & 63 & 0 & & 0 & 395575 & 1085888 \\
\hline $4 / 15 / 96$ & 21.5 & 2330 & 452220 & 1545 & 12663 & 0 & 6.12 & 63 & 3165 & & 0 & 398740 & 1085888 \\
\hline
\end{tabular}




\begin{tabular}{|c|c|c|c|c|c|c|c|c|c|c|c|c|c|}
\hline DATE & Pri. Level & Pri. Level & Pri/Sec & Pri Flow & Sec Flow & Sec Flow & Sec Level & Sec Level & Total Daily & Daily Rain & Daily Rain & Tot Accu & Tot Accu \\
\hline & inches & gallons & total flow & daily & total & daily & inches & gallons & Water-gal & inches & gallons & Water-gal & Rain-gal \\
\hline $4 / 16 / 96$ & 12.24 & 795 & 452760 & 540 & 12663 & 0 & 6.12 & 63 & $\begin{array}{r}-995 \\
\end{array}$ & 0.01 & 814.62 & 397745 & 1086703 \\
\hline $4 / 17 / 96$ & 11.65 & 735 & 453275 & 515 & 12663 & 0 & 6.16 & 64 & 456 & & 0 & 398201 & 1086703 \\
\hline $4 / 18 / 96$ & 11.97 & 770 & 453275 & 0 & 12663 & 0 & 6.17 & 65 & 36 & & 0 & 398237 & 1086703 \\
\hline $4 / 19 / 96$ & 11.8 & 750 & 454250 & 975 & 12663 & a) & 6.13 & 63 & 953 & & 0 & 399190 & 1086703 \\
\hline $4 / 20 / 96$ & 11.8 & 750 & 454250 & 0 & 12663 & 0 & 6.13 & 63 & 0 & & 0 & 399190 & 1086703 \\
\hline $4 / 21 / 96$ & 11.8 & 750 & 454250 & 0 & 12663 & 0 & 6.13 & 63 & 0 & & 0 & 399190 & 1086703 \\
\hline 4/22/96 & 17.43 & 1470 & 455470 & 1220 & 12663 & 0 & 6.14 & 64 & 1941 & 0.28 & 22809.36 & 401131 & 1109512 \\
\hline $4 / 23 / 96$ & 11.37 & 700 & 455680 & 210 & 12663 & a) & 6.15 & 64 & -560 & 0.02 & 1629.24 & 400571 & 1111142 \\
\hline $4 / 24 / 96$ & 13.64 & 940 & 456080 & 400 & 12663 & 0 & 6.17 & 65 & 641 & & 0 & 401212 & 1111142 \\
\hline 4/25/96 & 13.09 & 890 & 456700 & 620 & 12663 & 0 & 6.21 & 66 & 571 & & 0 & 401783 & 1111142 \\
\hline $4 / 26 / 96$ & 12.52 & 830 & 457750 & 1050 & 12663 & 0 & 6.17 & 65 & 989 & & 0 & 402772 & 1111142 \\
\hline $4 / 27 / 96$ & 12.52 & 830 & 457750 & 0 & 12663 & 0 & 6.17 & 65 & 0 & & 0 & 402772 & 1111142 \\
\hline 4/28/96 & 12.52 & 830 & 457750 & 0 & 12663 & 0 & 6.17 & 65 & 0 & & 0 & 402772 & 1111142 \\
\hline $4 / 29 / 96$ & 17.08 & 1420 & 457820 & 70 & 12663 & 0 & 6.18 & 65 & 660 & & 0 & 403432 & 1111142 \\
\hline $4 / 30 / 96$ & 11.68 & 740 & 459010 & 1190 & 12663 & 0 & 6.19 & 66 & 511 & & 0 & 403943 & 1111142 \\
\hline $5 / 1 / 96$ & 10.86 & 655 & 459310 & 300 & 12663 & 0 & 6.23 & 67 & 216 & & 0 & 404159 & 1111142 \\
\hline $5 / 2 / 96$ & 10.71 & 635 & 459960 & 650 & 12663 & 0 & 6.23 & 67 & 630 & & 0 & 404789 & 1111142 \\
\hline $5 / 3 / 96$ & 9.02 & 480 & 460332 & 372 & 12663 & 0 & 6.21 & 67 & 217 & & 0 & 405006 & 1111142 \\
\hline $5 / 4 / 96$ & 9.02 & 480 & 460332 & 0 & 12663 & 0 & 6.21 & 67 & 0 & & 0 & 405006 & 1111142 \\
\hline $5 / 5 / 96$ & 902 & 480 & 460332 & 0 & 12663 & 이 & 6.21 & 67 & 0 & & 0 & 405006 & 1111142 \\
\hline $5 / 6 / 96$ & 15.73 & 1230 & 460331 & -1 & 12663 & 이 & 6.21 & 67 & 149 & & 0 & 400100 & $111114<$ \\
\hline $5 / / / 96$ & 17.5 & 1490 & 460331 & 0 & 12663 & 0 & 6.26 & 69 & 262 & & 0 & 406017 & 1111142 \\
\hline $5 / 8 / 96$ & 19.17 & 1780 & 460340 & 9 & 12663 & 0 & 6.26 & 69 & 299 & & 0 & 406316 & 1111142 \\
\hline $5 / 9 / 96$ & 11.1 & 679 & 461891 & 1551 & 12663 & 0 & 6.23 & 67 & 448 & & 0 & 406764 & 1111142 \\
\hline $5 / 10 / 96$ & 9.42 & 517 & 462150 & 259 & 12663 & 0 & 6.27 & 70 & 100 & & 0 & 406864 & 1111142 \\
\hline $5 / 11 / 96$ & 9.42 & 517 & 462150 & 0 & 12663 & 0 & 6.27 & 70 & 0 & & 0 & 406864 & 1111142 \\
\hline $5 / 12 / 96$ & 9.42 & 517 & 462150 & 0 & 12663 & 0 & 6.27 & 70 & 0 & 0.14 & 11404.68 & 406864 & 1122546 \\
\hline $5 / 13 / 96$ & 16.04 & 1280 & 462160 & 10 & 12663 & 0 & 6.28 & 69 & 772 & & 0 & 407636 & 1122546 \\
\hline $5 / 14 / 96$ & 9.77 & 540 & 463436 & 1276 & 12663 & 0 & 6.28 & 69 & 536 & & 0 & 408172 & 1122546 \\
\hline $5 / 15 / 96$ & 9.77 & 540 & 463436 & 0 & 12663 & 0 & 6.28 & 69 & 0 & 0.06 & 4887.72 & 408172 & 1127434 \\
\hline $5 / 16 / 96$ & 12.75 & 855 & 463580 & 144 & 12663 & 0 & 6.28 & 69 & 459 & & 0 & 408631 & 1127434 \\
\hline $5 / 17 / 96$ & 12.75 & 855 & 463580 & 0 & 12663 & 0 & 6.28 & 69 & 0 & 0.12 & 9775.44 & 408631 & 1137210 \\
\hline $5 / 18 / 96$ & 12.75 & 855 & 463580 & 0 & 12663 & 0 & 6.28 & 69 & 0 & & 0 & 408631 & 1137210 \\
\hline $5 / 19 / 96$ & 12.75 & 855 & 463580 & 0 & 12663 & 0 & 6.28 & 69 & 0 & & 0 & 408631 & 1137210 \\
\hline $5 / 20 / 96$ & 6.93 & 310 & 464072 & 492 & 12663 & 0 & 6.29 & 69 & -53 & & 0 & 408578 & 1137210 \\
\hline $5 / 21 / 96$ & 9.81 & 543 & 465025 & 952 & 12664 & 1 & 6.37 & 72 & 1189 & 0.2 & 16292.4 & 409767 & 1153502 \\
\hline $5 / 22 / 96$ & 12.3 & 806 & 465025 & 0 & 12664 & 0 & 6.48 & 76 & 267 & & 0 & 410034 & 1153502 \\
\hline $5 / 23 / 96$ & 14.46 & 1053 & 465998 & 973 & 12664 & 0 & 6.48 & 76 & 1220 & & 0 & 411254 & 1153502 \\
\hline $5 / 24 / 96$ & 14.46 & 1053 & 465998 & 0 & 12664 & 0 & 6.48 & 76 & 0 & & 0 & 411254 & 1153502 \\
\hline
\end{tabular}


Trench 34

Sheet1

\begin{tabular}{|c|c|c|c|c|c|c|c|c|c|c|c|c|c|}
\hline DATE & Pri. Level & Pri. Level & Pri/Sec & Pri Flow & Sec Flow & Sec Flow & Sec Level & Sec Level & Total Daily & Daily Rain & Daily Rain & Tot Accu & Tot Accu \\
\hline & inches & gallons & total flow & daily & total & daily & inches & gallons & Water-gal & inches & gallons & Water-gal & Rain-gal \\
\hline $5 / 25 / 96$ & 14.46 & 1053 & 465998 & 0 & 12664 & 0 & 6.48 & 76 & 0 & & 0 & 411254 & 1153502 \\
\hline $5 / 26 / 96$ & 14.46 & 1053 & 465998 & 0 & 12664 & 0 & 6.48 & 76 & 0 & & 0 & 411254 & 1153502 \\
\hline $5 / 27 / 96$ & 14.46 & 1053 & 465998 & 0 & 12664 & 0 & 6.48 & 76 & 0 & & 0 & 411254 & 1153502 \\
\hline $5 / 28 / 96$ & 16.36 & 1323 & 465998 & 0 & 12664 & 0 & 6.73 & 85 & 279 & 0.01 & 814.62 & 411533 & 1154317 \\
\hline $5 / 29 / 96$ & 17.93 & 1546 & 465998 & 0 & 12664 & 0 & 6.79 & 85 & 223 & 0.09 & 7331.58 & 411756 & 1161648 \\
\hline $5 / 30 / 96$ & 17.93 & 1546 & 465998 & 0 & 12664 & 0 & 6.79 & 85 & 0 & & 0 & 411756 & 1161648 \\
\hline $5 / 31 / 96$ & 20.46 & 2010 & 465998 & 0 & 12664 & 0 & 6.92 & 94 & 473 & & 0 & 412229 & 1161648 \\
\hline $6 / 1 / 96$ & 20.46 & 2010 & 465998 & 0 & 12664 & 0 & 6.92 & 94 & 0 & & 0 & 412229 & 1161648 \\
\hline $6 / 2 / 96$ & 20.46 & 2010 & 465998 & 0 & 12664 & 0 & 6.92 & 94 & 0 & & 0 & 412229 & 1161648 \\
\hline $6 / 3 / 96$ & 23.29 & 2695 & 465998 & 0 & 12664 & 0 & 7.23 & 106 & 697 & & 0 & 412926 & 1161648 \\
\hline $6 / 4 / 96$ & 23.29 & 2695 & 465998 & 0 & 12664 & 0 & 7.23 & 106 & 0 & & 0 & 412926 & 1161648 \\
\hline $6 / 5 / 96$ & 25.31 & 3294 & 465998 & 0 & 12664 & 0 & 7.23 & 106 & 599 & & 0 & 413525 & 1161648 \\
\hline $6 / 6 / 96$ & 6.95 & 310 & 468604 & 2606 & 12664 & 0 & 7.42 & 114 & -370 & & 0 & 413155 & 1161648 \\
\hline $6 / 7 / 96$ & 6.95 & 310 & 468604 & 0 & 12664 & 0 & 7.42 & 114 & 0 & & 0 & 413155 & 1161648 \\
\hline $6 / 8 / 96$ & 6.95 & 310 & 468604 & 0 & 12664 & 0 & 7.42 & 114 & 0 & & 0 & 413155 & 1161648 \\
\hline $6 / 9 / 96$ & 6.95 & 310 & 468604 & 0 & 12664 & 0 & 7.42 & 114 & 0 & & 0 & 413155 & 1161648 \\
\hline $6 / 10 / 96$ & 6.95 & 310 & 468604 & 0 & 12664 & 0 & 7.42 & 114 & 0 & & 0 & 413155 & 1161648 \\
\hline $6 / 11 / 96$ & $6.9 \overline{5}$ & 310 & 468604 & 0 & 12664 & 0 & 7.42 & 114 & 0 & & 0 & 413155 & 1161648 \\
\hline $6 / 12 / 96$ & 19.02 & 1755 & 468604 & 0 & 12664 & 0 & 8.48 & 165 & 1496 & & 0 & 414651 & 1161648 \\
\hline c11910 & 20.20 & 1082 & A6gand & $n !$ & 136f4 & $n$ & 87 & 171 & 234 & & 0 & 414885 & 1161648 \\
\hline $6 / 14 / 96$ & 21.13 & 2145 & 468604 & 0 & 12664 & 0 & 8.87 & 176 & 167 & & 0 & 415052 & 1161648 \\
\hline $6 / 15 / 96$ & 21.13 & 2145 & 468604 & 0 & 12664 & 0 & 8.87 & 176 & 0 & & 0 & 415052 & 1161648 \\
\hline $6 / 16 / 96$ & 21.13 & 2145 & 468604 & 0 & 12664 & 0 & 8.87 & 176 & 0 & & 0 & 415052 & 1161648 \\
\hline $6 / 17 / 96$ & 21.13 & 2145 & 468604 & 0 & 12664 & 0 & 8.87 & 176 & 0 & & 0 & 415052 & 1161648 \\
\hline $6 / 18 / 96$ & 24.61 & 3062 & 468604 & 0 & 12664 & 0 & 9.89 & 234 & 975 & & 0 & 416027 & 1161648 \\
\hline $6 / 19 / 96$ & & & & & & & & & & & & & \\
\hline
\end{tabular}


WHC-SI)-WM-TI-767, Rev. 0

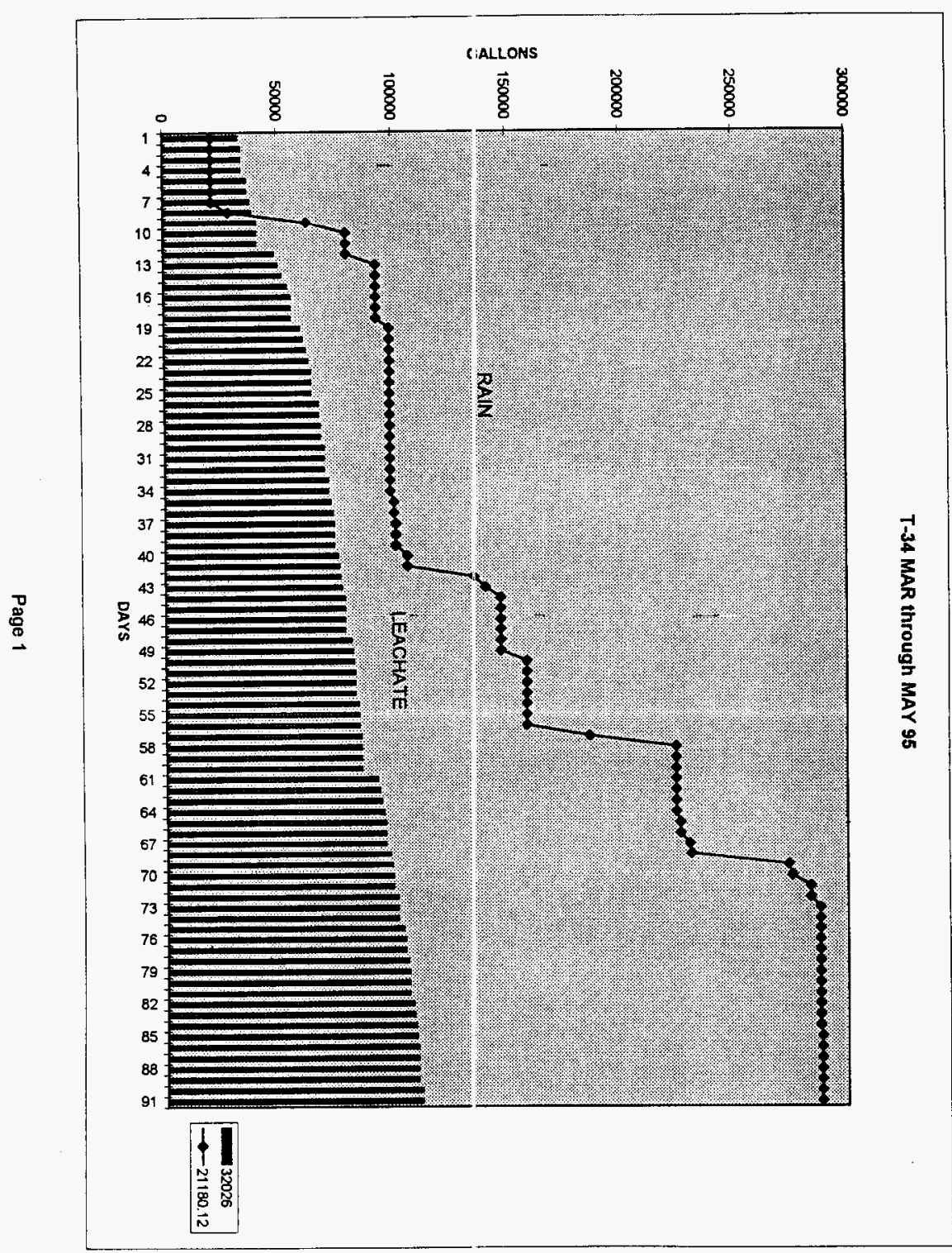


WHC-Si)-WM-T1-767, Rev. 0

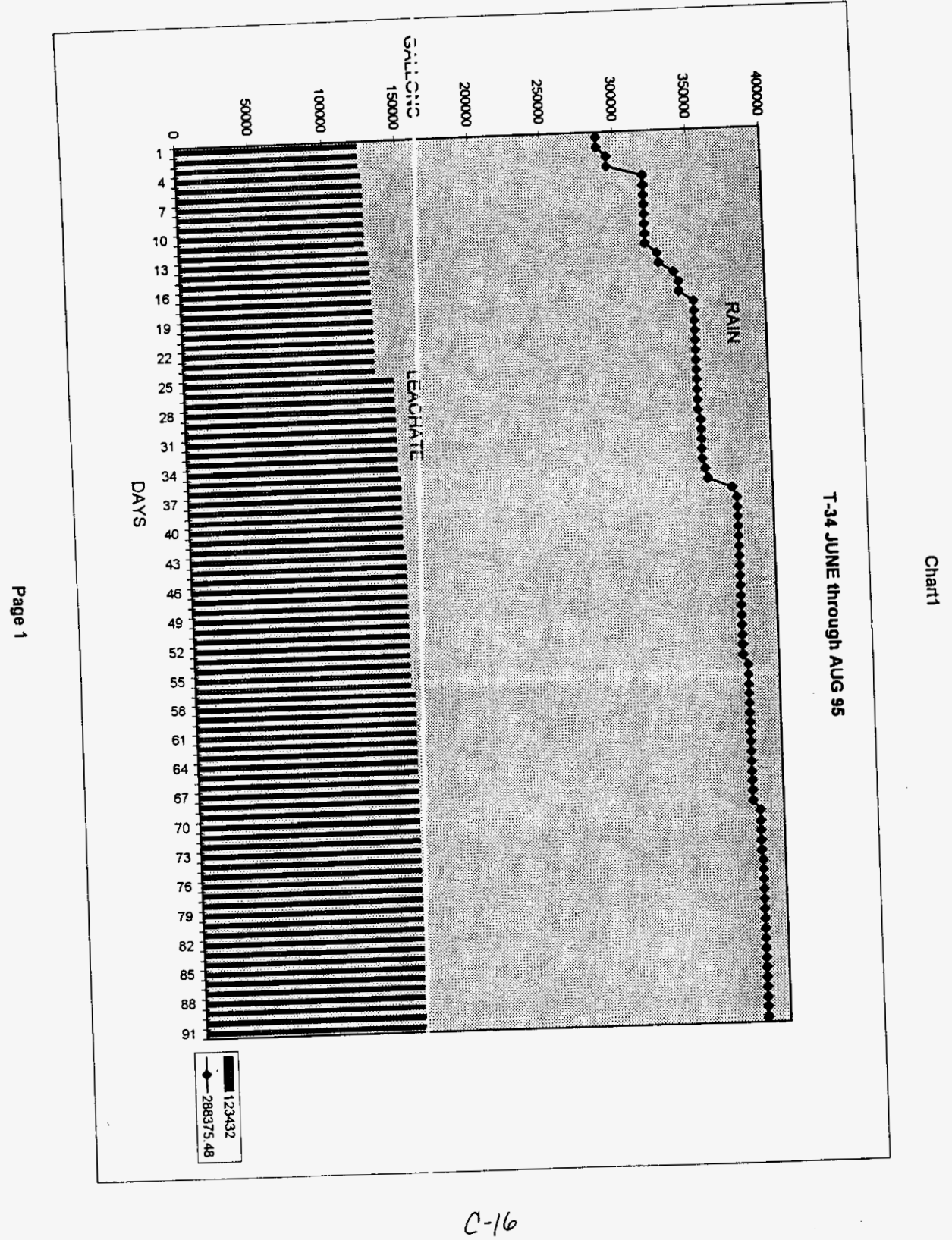


WHC-:SD-WM-TI-767, Rev. 0

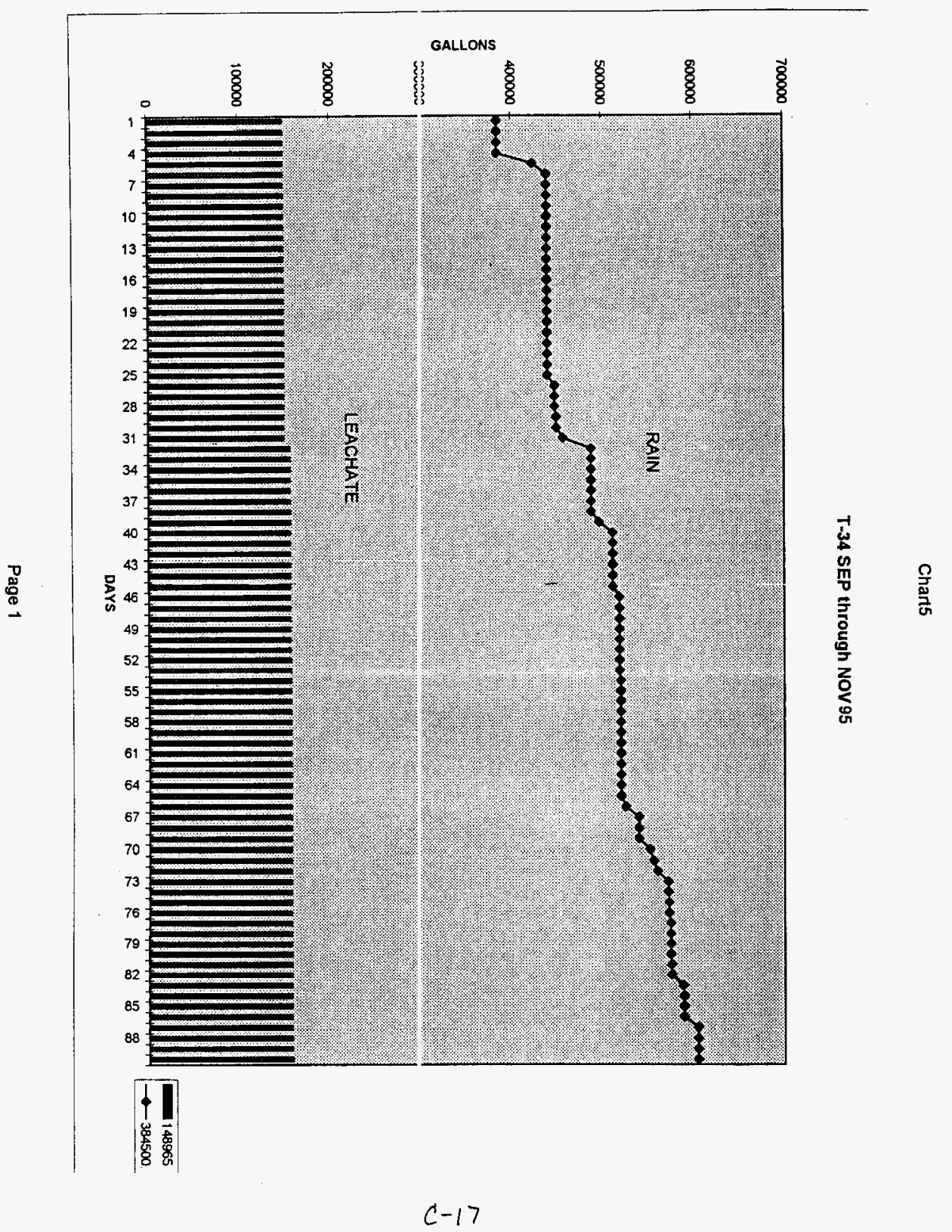


WHC-S )-WM-TI-767, Rev. 0

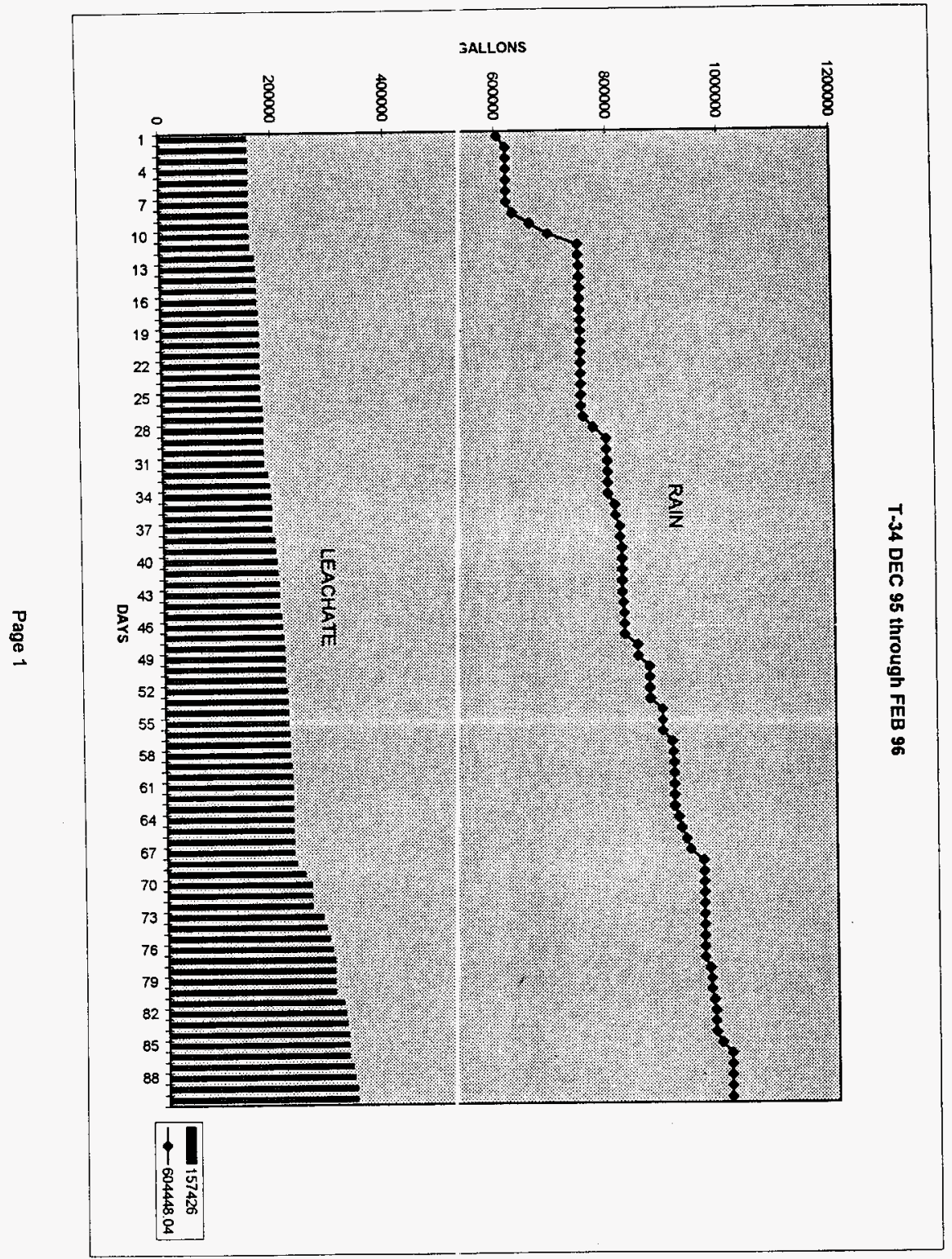




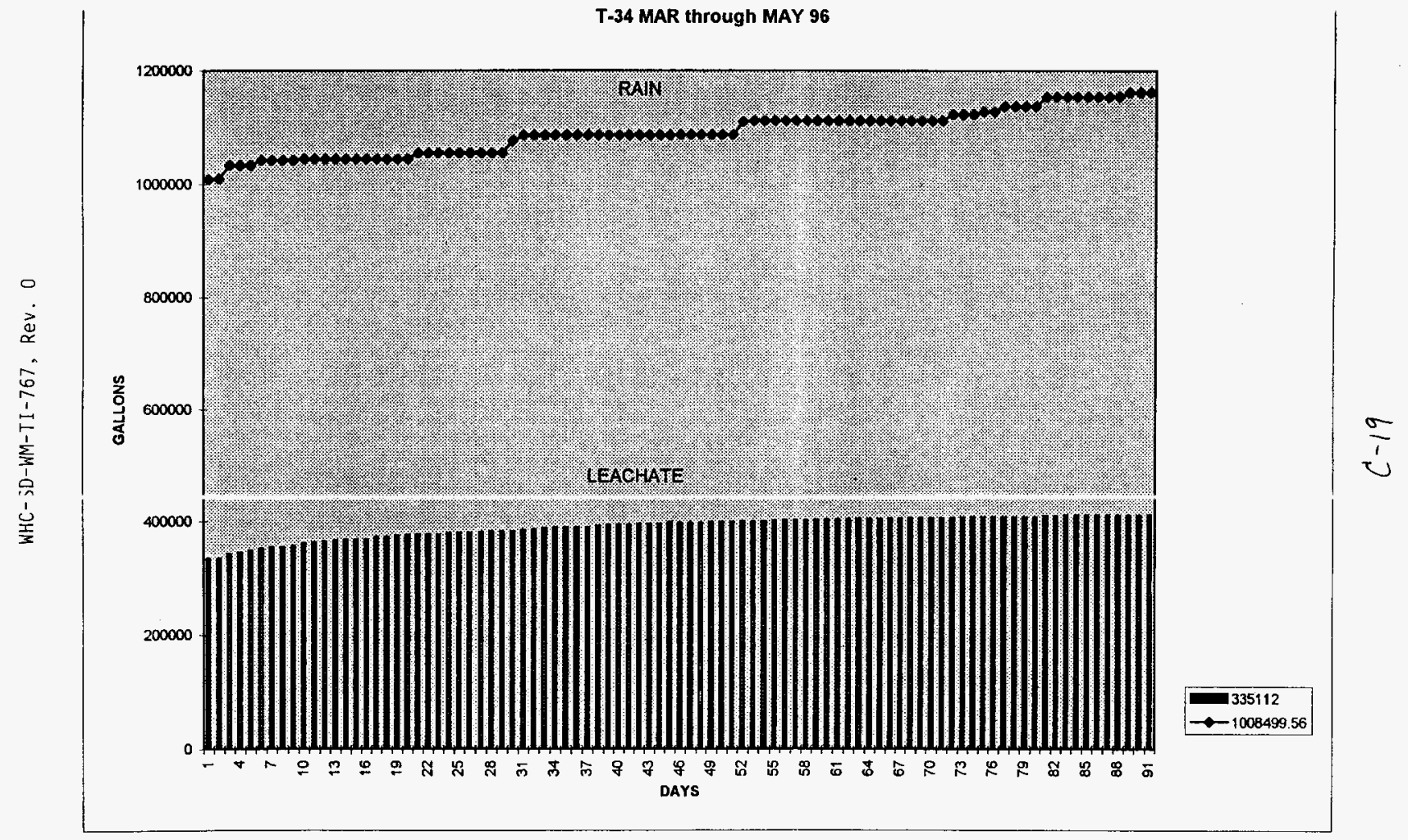

